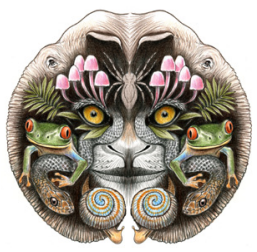

ISSN 0974-7907 (Online) ISSN 0974-7893 (Print)

OPEN ACCESS

\title{
A CHECKLIST OF FISHES OF KERALA, INDIA
}

\section{A. Bijukumar ${ }^{1} \&$ Rajeev Raghavan ${ }^{2}$}

${ }^{1}$ Department of Aquatic Biology and Fisheries, University of Kerala, Thiruvananthapuram, Kerala 695581, India ${ }^{2}$ Center for Taxonomy of Aquatic Animals, Department of Fisheries Resource Management, Kerala University of Fisheries and Ocean Studies (KUFOS), Kochi, Kerala 682506, India ${ }^{2}$ Laboratory of Systematics, Ecology and Conservation, Zoo Outreach Organization (ZOO), Coimbatore, Tamil Nadu 641035, India ${ }^{1}$ bijupuzhayoram@gmail.com (corresponding author), ${ }^{2}$ rajeevraq@hotmail.com

Abstract: A checklist of the fishes of Kerala State is presented, along with their scientific and common names (English and Malayalam), endemism, IUCN Red List status, listing under different Schedules of the Indian Wildlife (Protection) Act and in the Appendices of Convention on International Trade in Endangered Species of Wild Flora and Fauna (CITES). Nine Hundred and five species of fishes are recorded from the inland and marine waters of Kerala comprising of 41 orders and 172 families. Close to $30 \%$ of the freshwater fish species found in Kerala are endemic to the State. Only $8 \%$ of the tota fishes of Kerala are listed as threatened in the IUCN Red List, of which the majority are freshwater species. Several hundred fish species occurring in the marine waters of Kerala have not yet been assessed for their conservation status by IUCN.

Keywords: Brackishwater, fish, freshwater, Kerala, marine, species.

Fish diversity known from the fresh and marine waters of India constitutes $9.7 \%$ of the total number ( 33,059 species) of fish species known from the world (Eschmeyer \& Fong 2014). Of these, marine fishes alone account for $7.4 \%$. An assessment of the ichthyodiversity of India recognizes an estimated 3,231 valid species of freshwater and marine fishes, of which the total diversity of marine species comprise $75.6 \%$ (2,443 species) (Gopi \& Mishra 2015).

Despite over 200 years of exploration and research, the fish fauna of peninsular India and Kerala continue to be poorly known. Freshwater ichthyological research in Kerala started in the late $18^{\text {th }}$ century with the description of Cirrhinus cirrhosus and Labeo fimbriatus by Bloch (1795) from the erstwhile Malabar, followed by the description of Wallago attu from the same region by Bloch \& Schneider (1801). Over the next century (1800-1900), several naturalists including Georges Cuvier (1828-1831), Achilles Valenciennes (1840-1847), Thomas Jerdon (1849), Albert Günther (1864) and Francis Day (1865-1889) advanced the knowledge of freshwater fishes of erstwhile Malabar District and Cochin State. One of the earliest checklists of freshwater fishes of the
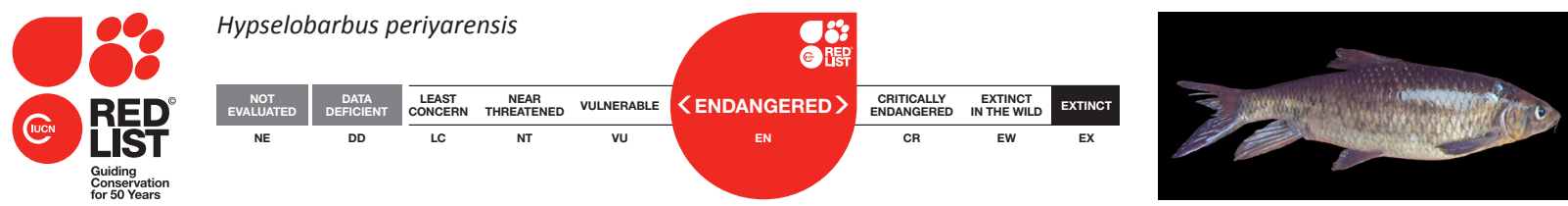

DOI: http://dx.doi.org/10.11609/jott.2004.7.13.8036-8080 | ZooBank: urn:Isid:zoobank.org:pub:624B60B8-E050-4C63-BEA4-85B67DEAEB75

Editor: Mewa Singh, University of Mysore, Mysuru, India

Date of publication: 17 November 2015 (online \& print)

Manuscript details: Ms \# 04308 | Received 11 May 2015 | Final received 23 September 2015 | Finally accepted 29 September 2015

Citation: Bijukumar, A. \& R. Raghavan (2015). A checklist of fishes of Kerala, India. Journal of Threatened Taxa 7(13): 8036-8080; http://dx.doi.org/10.11609/ jott.2004.7.13.8036-8080

Copyright: () Bijukumar \& Raghavan 2015. Creative Commons Attribution 4.0 International License. JoTT allows unrestricted use of this article in any medium, reproduction and distribution by providing adequate credit to the authors and the source of publication.

Funding: Rajeev Raghavan thanks the Critical Ecosystem Partnership Fund (CEPF), Western Ghats Program and the Mohammed Bin Zayed Species Conservation Fund for funding various projects during which the data for the checklist was compiled.

Conflict of Interest: The authors declare no competing interests.
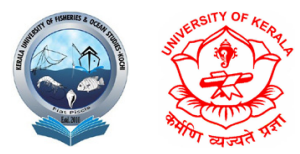

Acknowledgements: Rajeev Raghavan thanks Neelesh Dahanukar, J.D. Marcus Knight, Siby Philip, Anvar Ali and Unmesh Katwate for providing necessary help and suggestions during the compilation of this checklist. 
region - On the fresh water fishes of southern India was published by Jerdon (1849), in which he gave an account of the fishes of Madras Presidency (including Malabar State).

Francis Day, a medical surgeon of the British army, who later turned to ichthyology, laid the foundation for fisheries research in Kerala. His book The Fishes of Malabar (Day 1865a) was the first comprehensive account on the diversity of fishes off the Kerala coast. He describes that "while staying at Cochin on the Malabar Coast of India, from 1859 to 1864, the annually increasing importance of its fisheries came most prominently to my notice. Desirous of ascertaining what species of fish were most abundant, and which were best adapted for different manufacturers, I was induced to make a collection of those procurable from the sea, estuaries or freshwaters, with notes on their habits and uses, to elucidate which, plain and coloured drawings of each species were made as soon as possible after the fish had been captured". Francis Day subsequently published pioneering accounts on the fishes of the region including On the Fishes of Cochin, on the Malabar Coast of India (Day 1865b,c), On the Fishes of the Neilgherry Hills and Rivers around their Bases (Day 1867a) and On some Fishes from the Wynaad (Day 1867b).

The H.M.S. Challenger Expedition in the late sixties and seventies of the $19^{\text {th }}$ century triggered interest in marine resource surveys in the Indian seas and recorded the occurrence of several marine fishes along the Kerala coast. Taxonomic investigations on deep-sea fishes of India began with the publications of A. Alcock, based on the samples collected during the voyage of Indian marine survey steamer H.M.S. Investigator (Alcock 1899); and the descriptions also include a few deepsea fishes off the erstwhile Travancore State. In the $20^{\text {th }}$ century, several ichthyologists compiled information on marine fish fauna of the Indian Ocean, including those in the western ocean, notable in this series being The Fishes of Indo-Australian Archipelago (Weber \& de Beaufort, 1916-1936; de Beaufort 1940; de Beaufort \& Chapman 1951).

The freshwater fishes of Travancore region remained poorly known until the checklists of Pillai (1929), Hora \& Law (1941), Raj (1941) and Silas (1951) were published. During the last 50 years, several localized checklists and compilations confined to smaller regions such as a district, a protected area or a river system of Kerala have been published.

Until this day, very few comprehensive checklists (Chhapgar \& Manakadan 2000; Gopi 2000; Kurup et al. 2004; Bijukumar et al. 2010) have been published on the fishes of Kerala State. Since the publication of the most recent comprehensive checklist (Kurup et al. 2004), around 20 new freshwater species have been described, in addition to revalidation of the species status of several others. There have also been many new genera and a new family of freshwater fish identified from Kerala during this period (2004-2014). The last one decade witnessed renewed interest in marine fish taxonomy, with many new species described from the Kerala coast and more records of deep-sea fishes from the country's exclusive economic zone of India. Recent information compiled by Gopi \& Mishra (2015) reveals that 2,443 species of marine fish are recognized from India, represented under 230 families and 927 genera; though state-wise species records and list of species were not mentioned.

The last two years has also witnessed an increasing trend of dubious 'taxonomic' practices creeping into the ichthyological literature of Kerala State thereby disrupting an otherwise renewed phase in freshwater fish taxonomy in the region (see Raghavan et al. 2014). For the purpose of the present checklist, we have not considered any description that has appeared in a 'predatory journal' in which the peer review is compromised (see Beall 2012, 2015; Raghavan et al. 2014). In this checklist, freshwater fishes are listed in brown colored font. The column marked EC (Ecosystem) denotes whether the habitat of the species is marine (M), freshwater (F) or primarily marine but secondary freshwater (S).

\section{REFERENCES}

Alcock, A. (1899). A descriptive catalogue of the Indian deep-sea fishes in the Indian Museum. Being a revised account of the deep-sea fishes collected by the Royal Indian marine survey ship Investigator. Indian Museum, Calcutta, 211pp.

Beall, J. (2015). Scholarly Open Access - critical analysis of scholarly open-access publishing. http://scholarlyoa.com/. Accessed on 14 April 2015.

Beall, J. (2012). Predatory publishers are corrupting open access. Nature 489: 179; http://dx.doi.org/10.1038/489179a

Bijukumar, A., T.K. Sirajudheen, L. Rajesh \& T. Radhakrishnan (2010). Marine fish and fisheries of Kerala- Heritage, diversity and conservation paradigms, pp. 32-102. In: Leonard, S. \& A. Benizger (eds.). Conservation of Fishes in Kerala, India. World Aquarium, St. Louis, USA.

Bloch, M.E. (1795). Naturgeschichte der ausländischen Fische. Berlin. v. 9: i-ii + 1-192, pls. 397-429.

de Beaufort, L.F. (1940). The Fishes of Indo-Australian Archipelago. E. J. Brill, Leiden 8, 508pp.

Bloch, M.E. \& J.G. Schneider (1801). M.E. Blochii, Systema Ichthyologiae Iconibus cx Ilustratum. Post obitum auctoris opus inchoatum absolvit, correxit, interpolavit Jo. Gottlob Schneider, Saxo. Berolini. Sumtibus Auctoris Impressum et Bibliopolio Sanderiano Commissum. i-Ix + 1-584, Pls. 1-110pp.

Chhapgar, B.F. \& R. Manakadan (Eds). (2000). Ecology of Hill Streams 


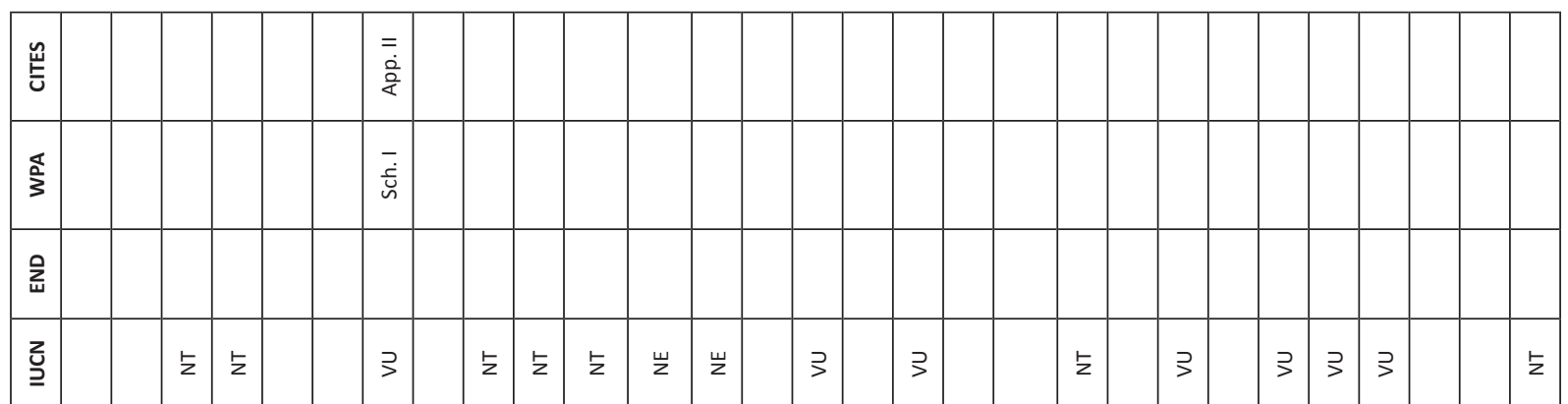

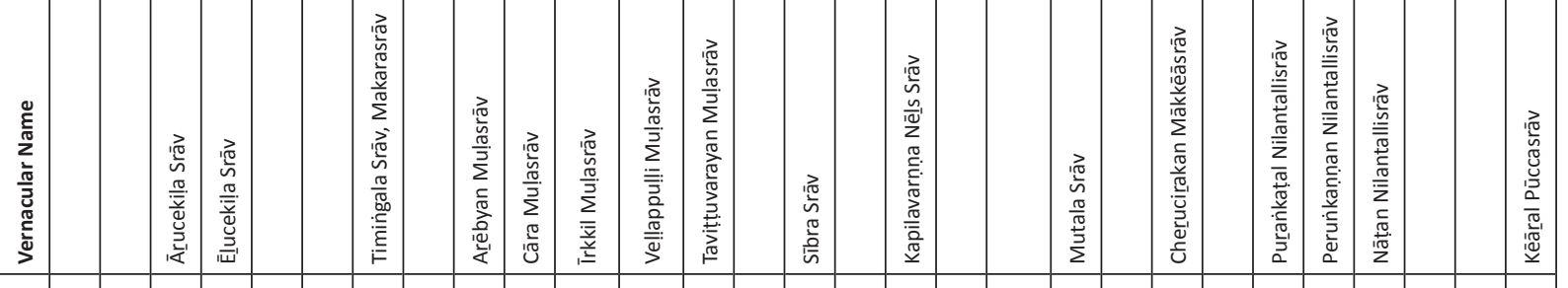

\begin{tabular}{|c|c|c|c|c|c|c|c|c|c|c|c|c|c|c|c|c|}
\hline 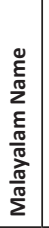 & 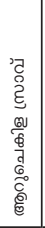 & 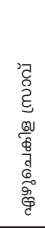 & 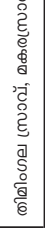 & 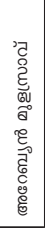 & 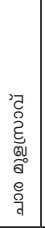 & 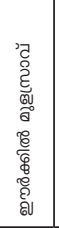 & 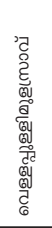 & 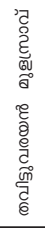 & 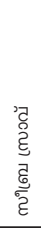 & 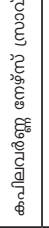 & 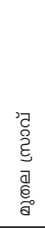 & 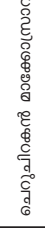 & 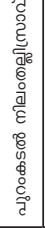 & $\frac{\square}{6}$ & 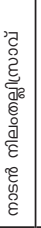 & 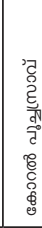 \\
\hline $\begin{array}{l}\frac{z}{2} \\
\overline{0} \\
\frac{+}{a}\end{array}$ & 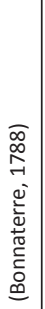 & 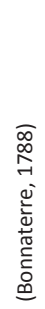 & 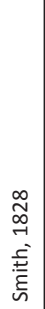 & 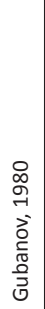 & 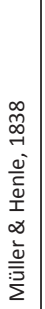 & 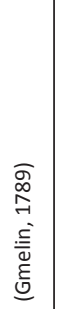 & 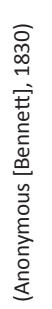 & 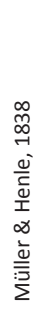 & 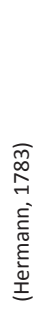 & 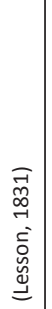 & 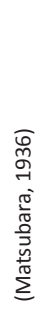 & 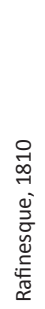 & 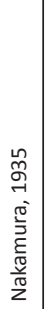 & 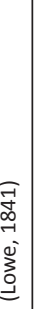 & 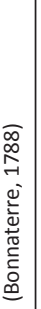 & 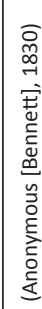 \\
\hline
\end{tabular}

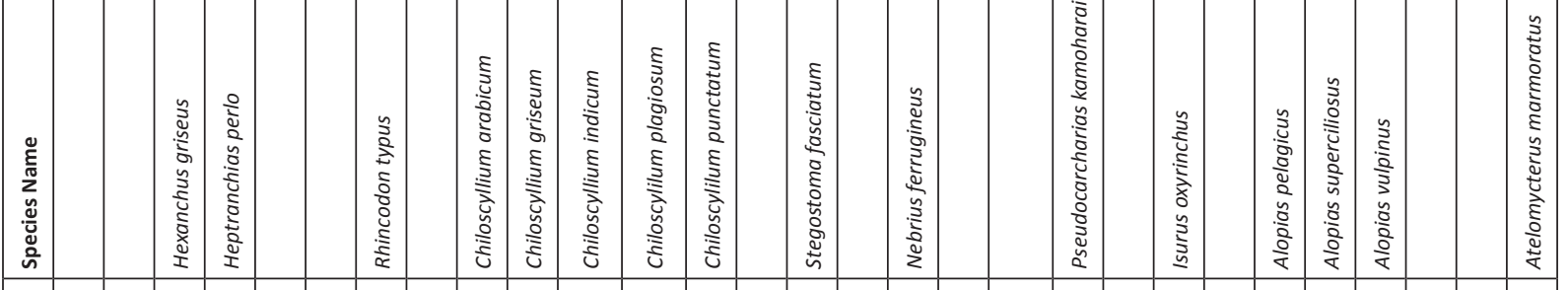

\begin{tabular}{|c|c|c|c|c|c|c|c|c|c|c|c|c|c|c|c|c|c|c|c|c|c|c|c|c|c|c|c|c|}
\hline 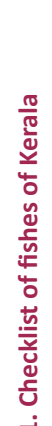 & 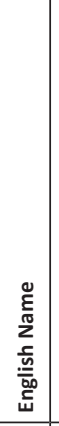 & 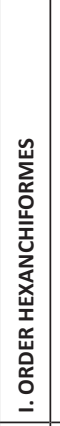 & 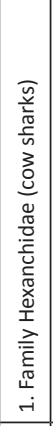 & 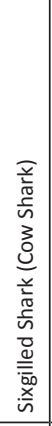 & 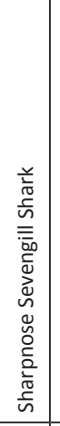 & 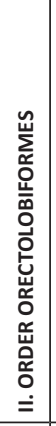 & 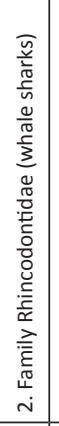 & \begin{tabular}{|l}
$\mid$ \\
\\
\\
0 \\
0 \\
0 \\
0 \\
0
\end{tabular} & 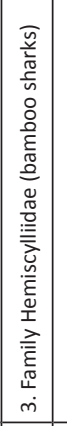 & 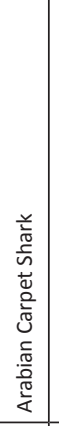 & 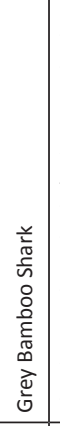 & 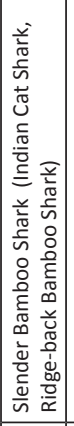 & 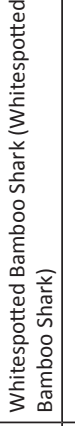 & 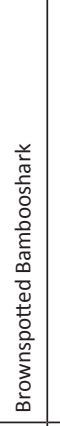 & 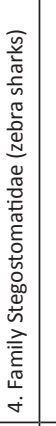 & 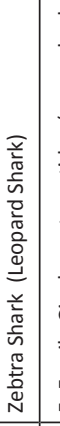 & 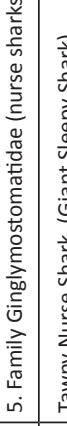 & 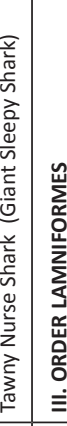 & 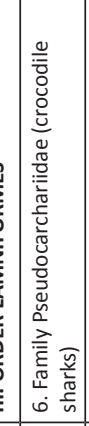 & 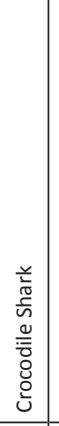 & 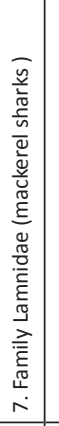 & 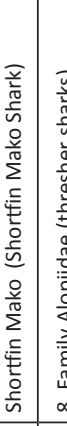 & 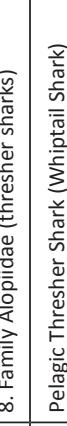 & 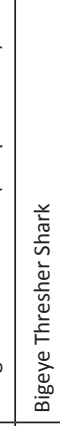 & 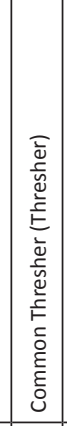 & 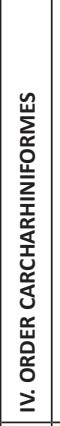 & 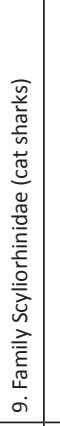 & 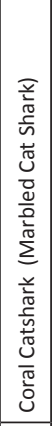 \\
\hline & & & & $\neg$ & $\sim$ & & & $m$ & & $\sigma$ & in & 0 & n & $\infty$ & & $\sigma$ & & 욕 & & $\exists$ & & $\approx$ & $m$ & $\vec{J}$ & $\stackrel{\text { ص }}{2}$ & & & $\stackrel{\sim}{-1}$ \\
\hline
\end{tabular}




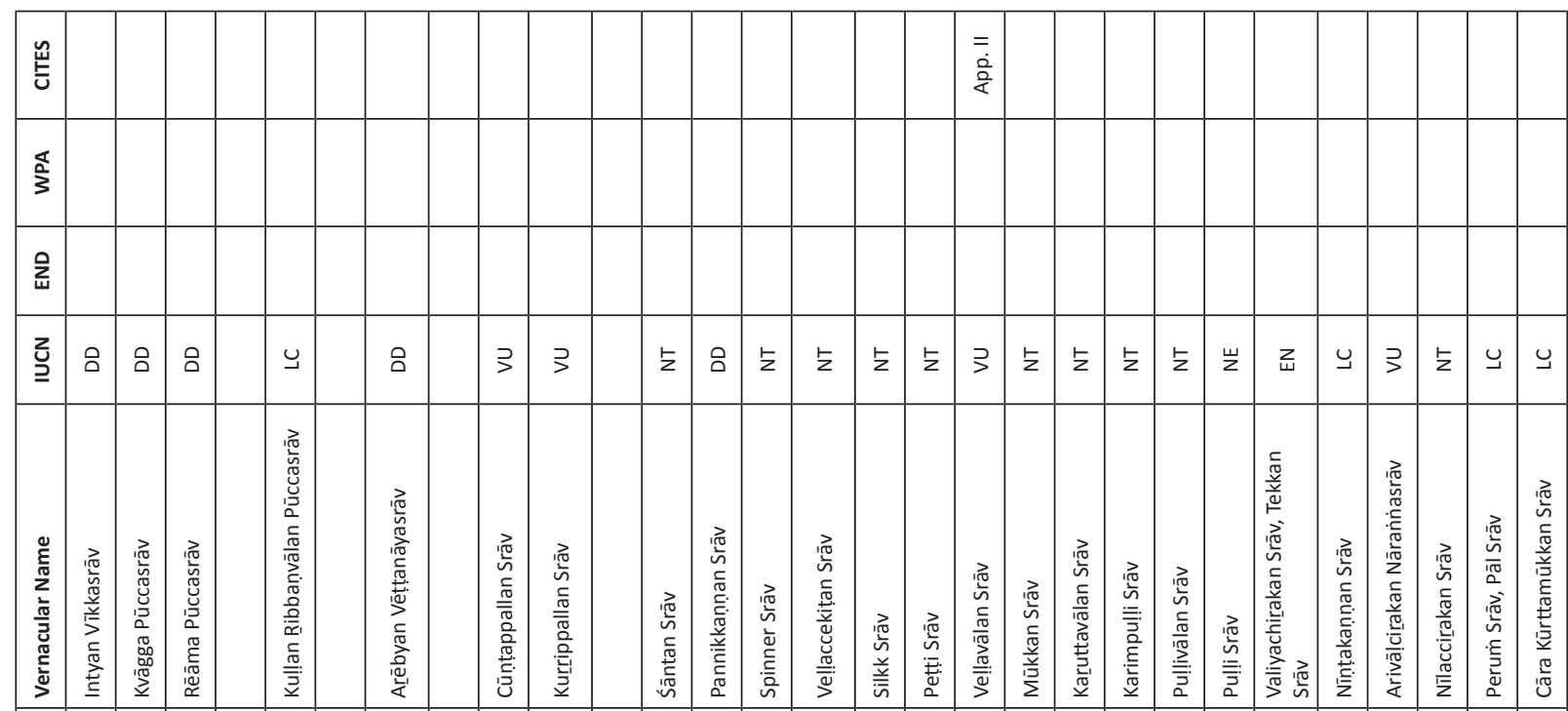

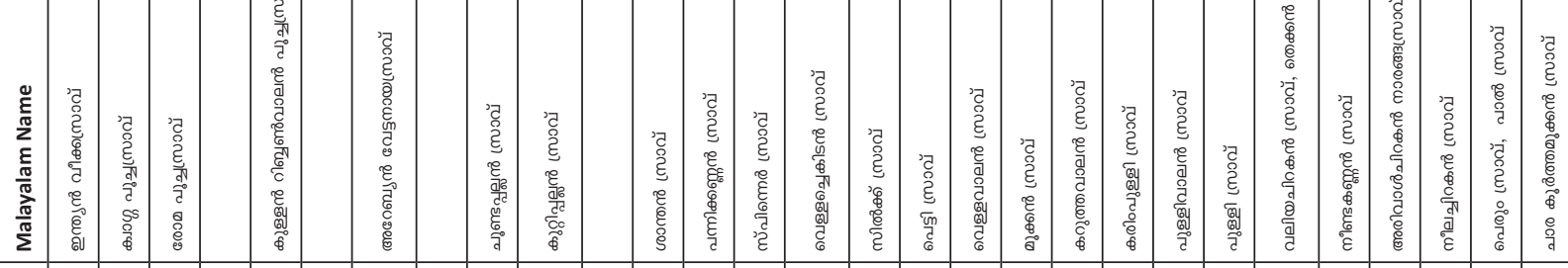

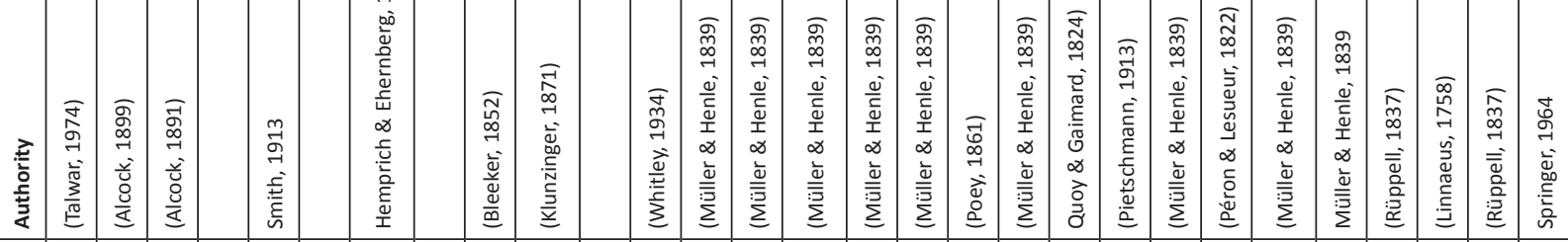

\begin{tabular}{|c|c|c|c|c|c|c|c|c|c|c|c|c|c|c|c|c|c|c|c|c|c|c|c|c|c|}
\hline & 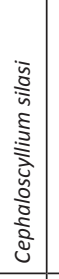 & 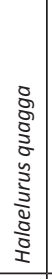 & 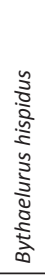 & & 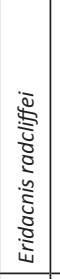 & & 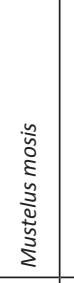 & & & 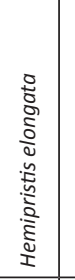 & 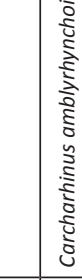 & 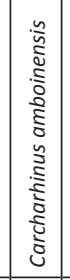 & 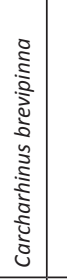 & 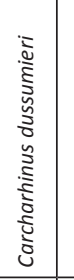 & 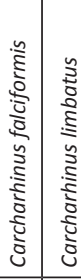 & 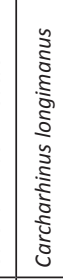 & 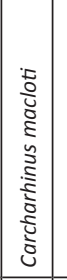 & 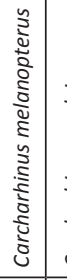 & 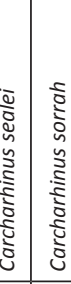 & 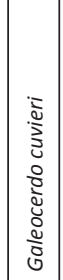 & 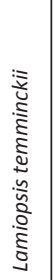 & & 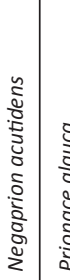 & 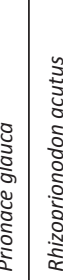 & \\
\hline & 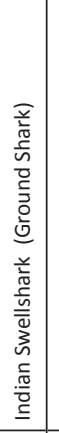 & 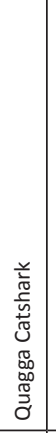 & 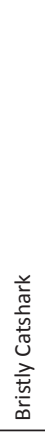 & 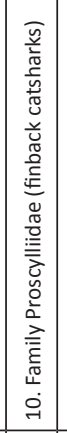 & 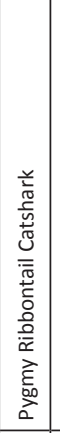 & 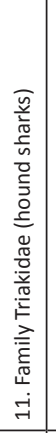 & 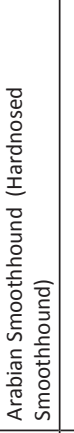 & 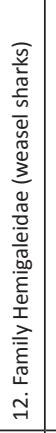 & 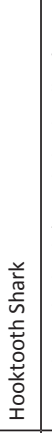 & 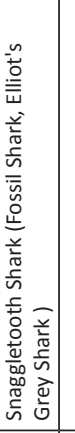 & 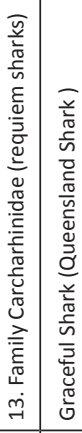 & 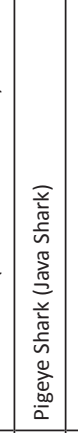 & 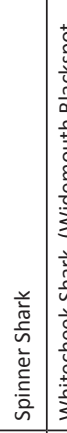 & 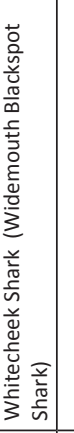 & 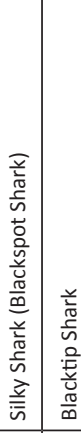 & 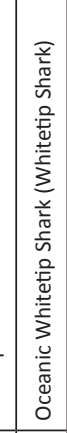 & 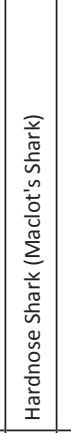 & 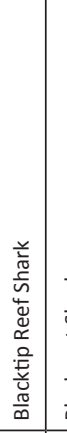 & 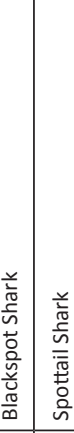 & 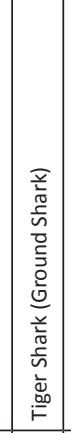 & 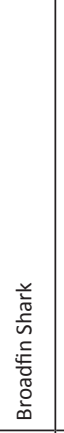 & 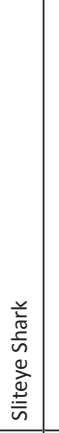 & 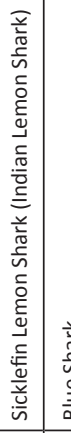 & & 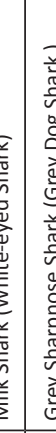 \\
\hline & 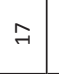 & $\stackrel{\infty}{\rightarrow}$ & g & & $\stackrel{\sim}{\sim}$ & & $\vec{\lambda}$ & & $\approx$ & 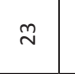 & $\stackrel{\sim}{\sim}$ & $\stackrel{\sim}{\sim}$ & $\stackrel{\sim}{N}$ & $\hat{\sim}$ & \begin{tabular}{l|l}
$\sim$ & $\stackrel{\sim}{~}$
\end{tabular} & 品 & $\vec{m}$ & $\approx$ & $\stackrel{m}{m}$ & 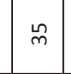 & $\stackrel{m}{\infty}$ & $\hat{m}$ & $\stackrel{\infty}{m}$ & ळి & \\
\hline
\end{tabular}




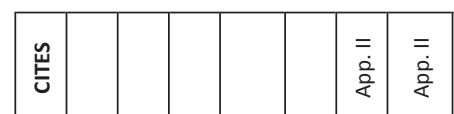

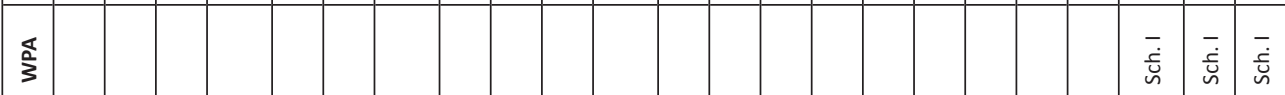

㩊

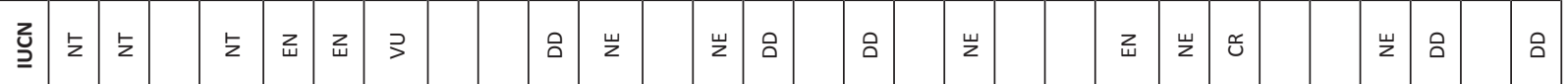

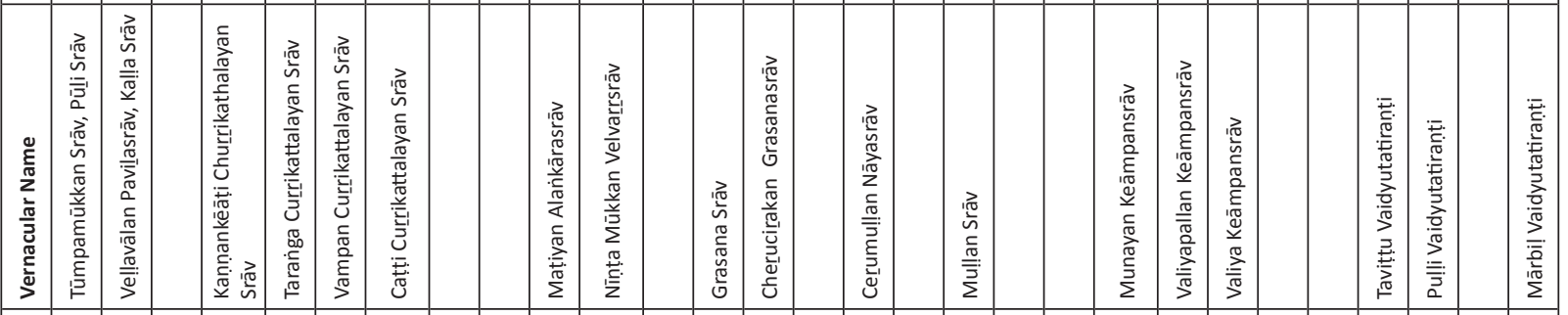

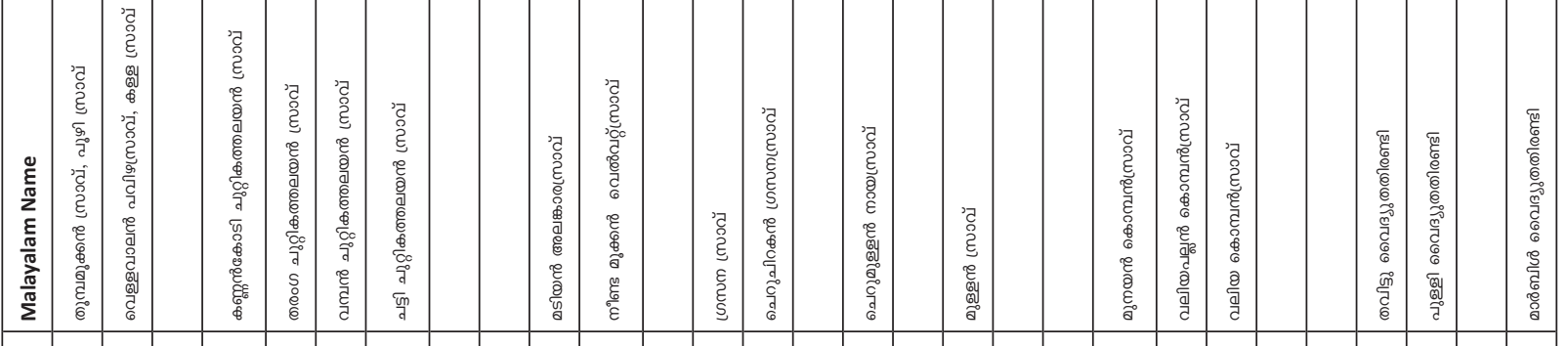

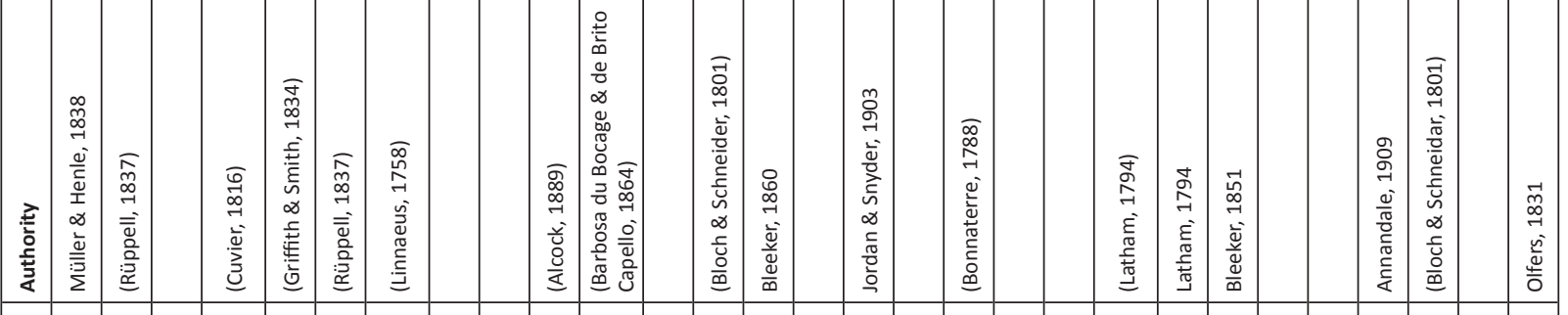

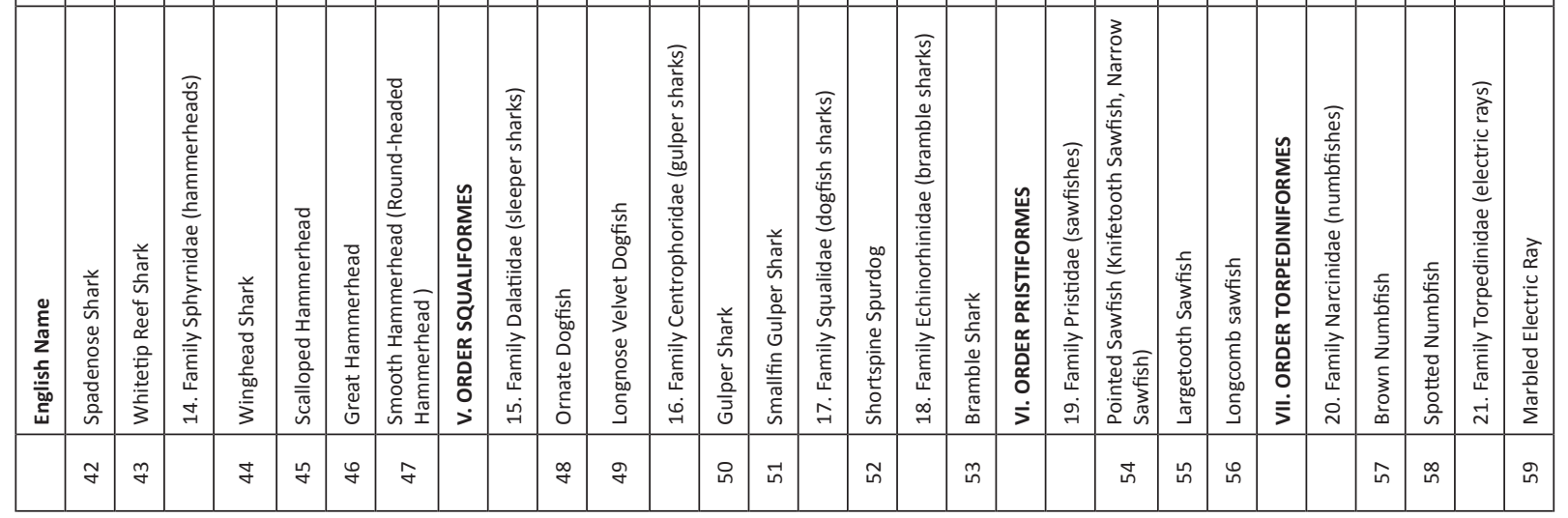




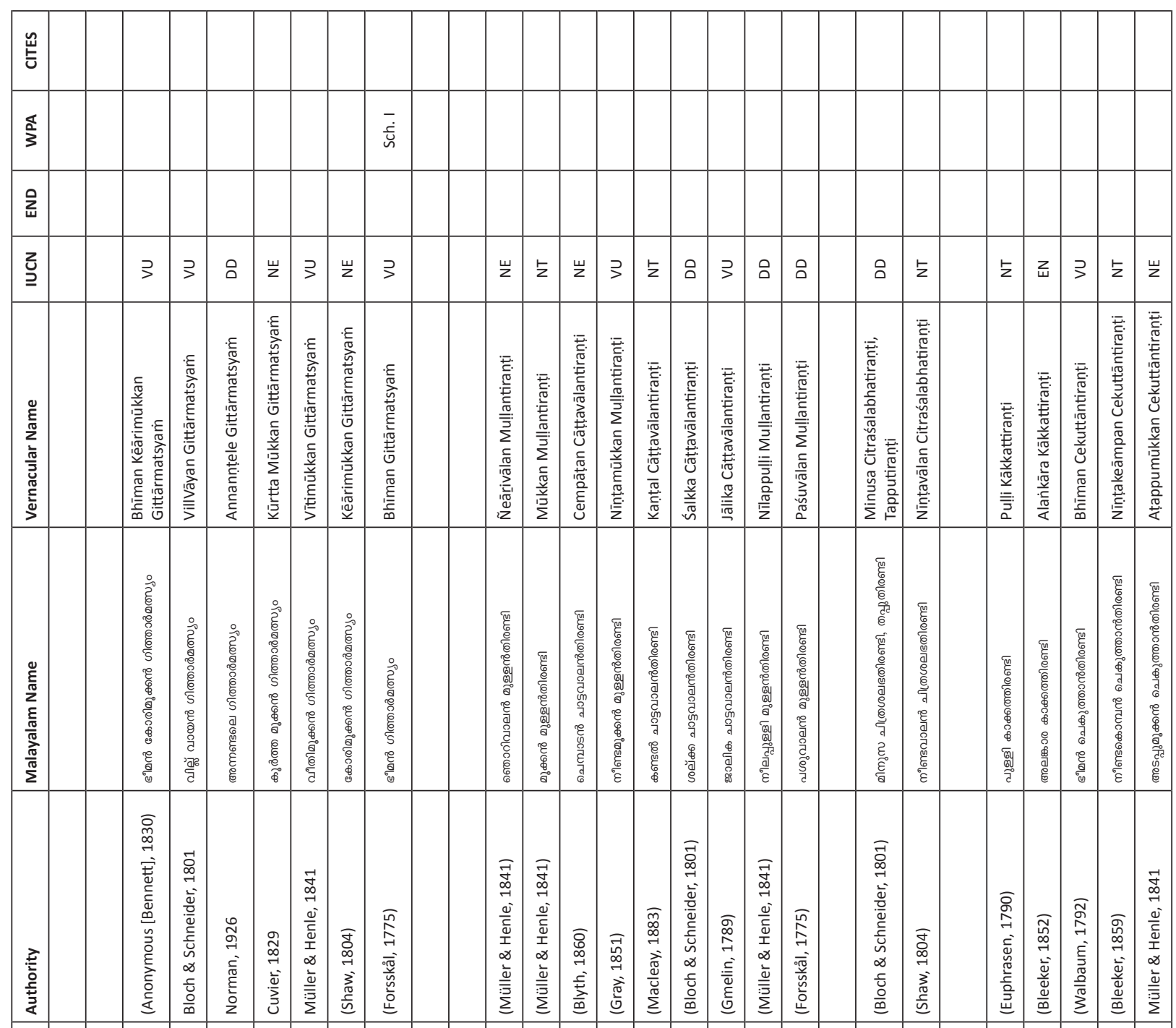

\begin{tabular}{|c|c|c|c|c|c|c|c|c|c|c|c|c|c|c|c|c|c|c|c|c|c|c|c|c|c|c|c|}
\hline & & & 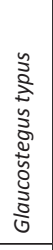 & 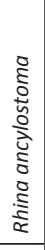 & 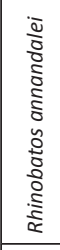 & 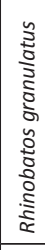 & 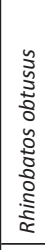 & 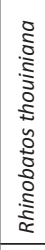 & 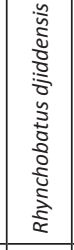 & & & & 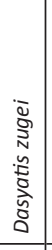 & 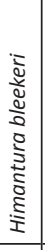 & 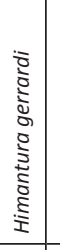 & 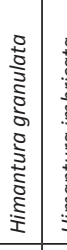 & 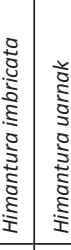 & 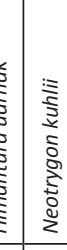 & 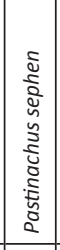 & & 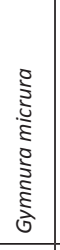 & 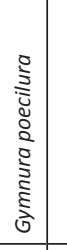 & & 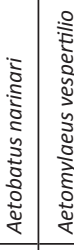 & 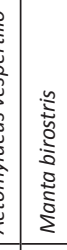 & 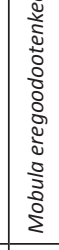 & . \\
\hline & 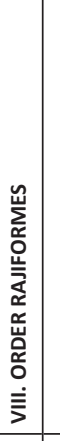 & 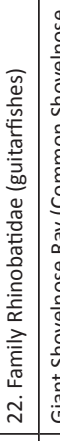 & 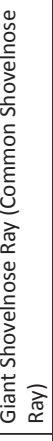 & 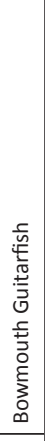 & 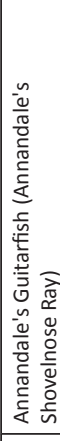 & 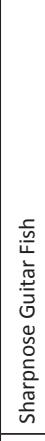 & 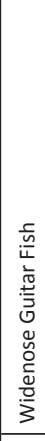 & 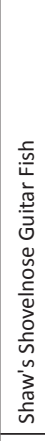 & 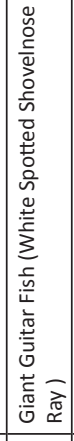 & 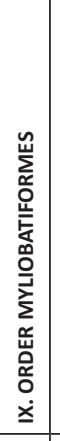 & 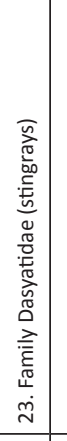 & 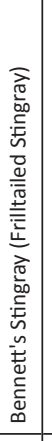 & 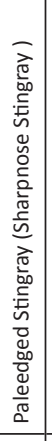 & 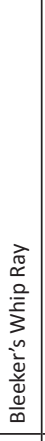 & 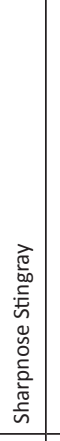 & 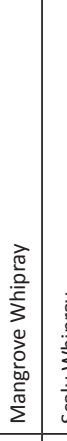 & 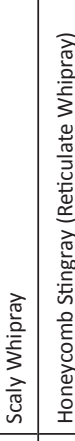 & 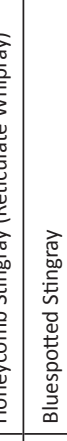 & 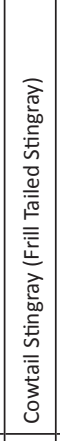 & 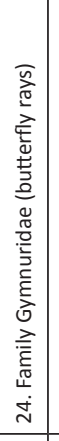 & 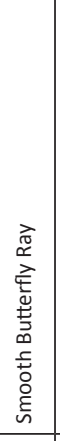 & $\begin{array}{l} \\
\\
\\
y\end{array}$ & 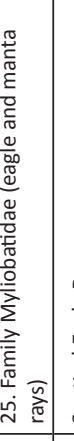 & 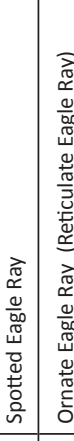 & 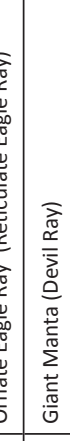 & 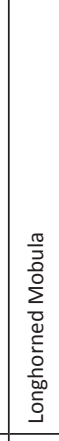 & \\
\hline & & & & 5 & $\tilde{\sigma}$ & ஜ) & to & น & $\stackrel{8}{\circ}$ & & & 6 & $\stackrel{\infty}{\infty}$ & 8 & $\curvearrowright$ & $\vec{\lambda}$ & $\approx$ & \begin{tabular}{l|l}
$R$ \\
$R$
\end{tabular} & $\stackrel{n}{n}$ & & $\stackrel{\circ}{\wedge}$ & $\approx$ & & 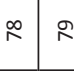 & $\begin{array}{lll}8 & \infty\end{array}$ & $\vec{\infty}$ & \\
\hline
\end{tabular}




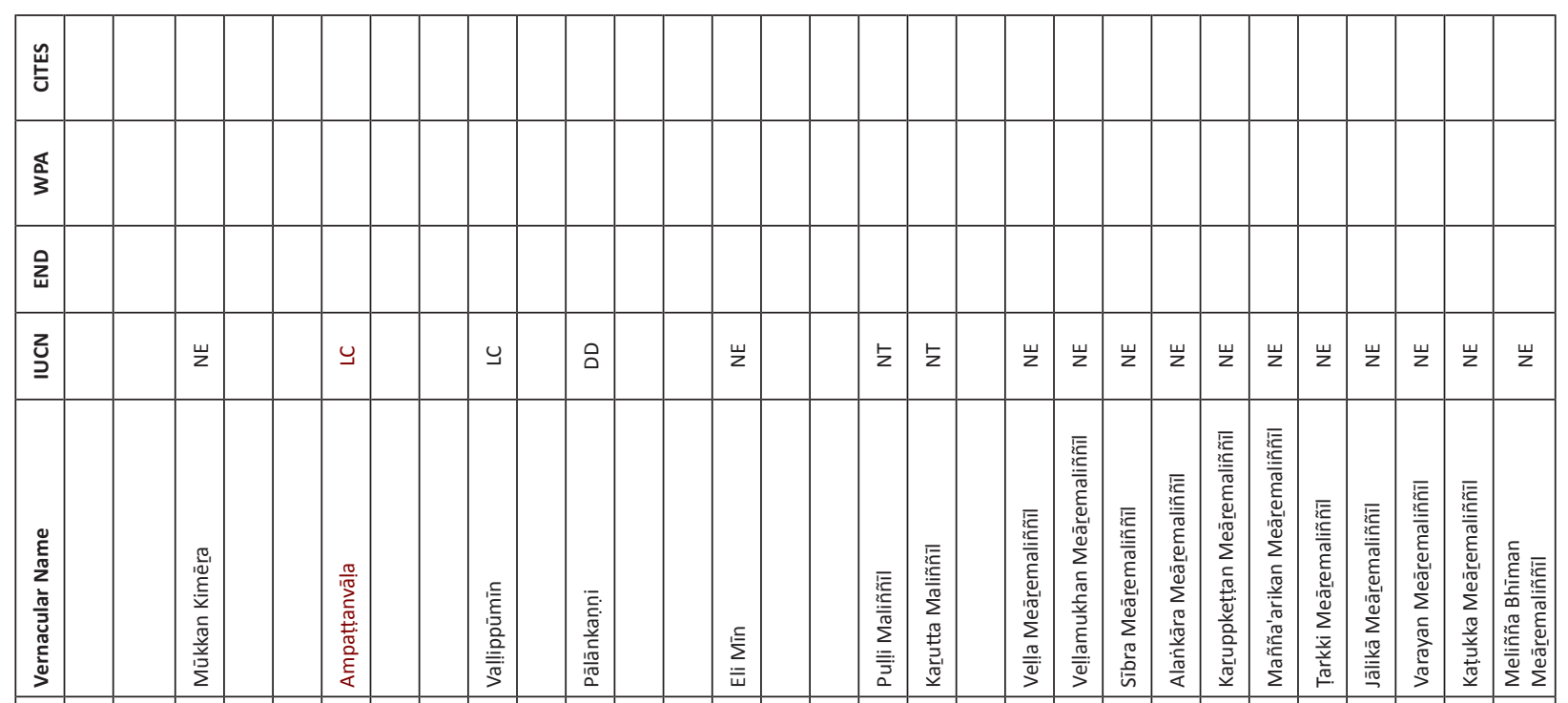

\begin{tabular}{|c|c|c|c|c|c|c|c|c|c|c|c|c|c|c|c|}
\hline 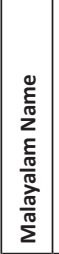 & 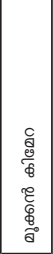 & 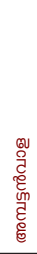 & 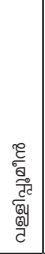 & 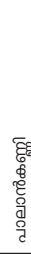 & 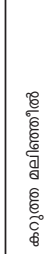 & 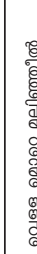 & 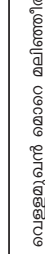 & 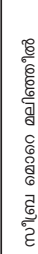 & 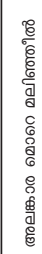 & 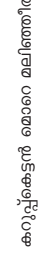 & 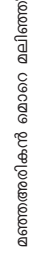 & 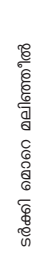 & 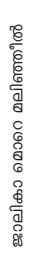 & 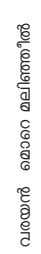 & 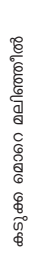 \\
\hline
\end{tabular}

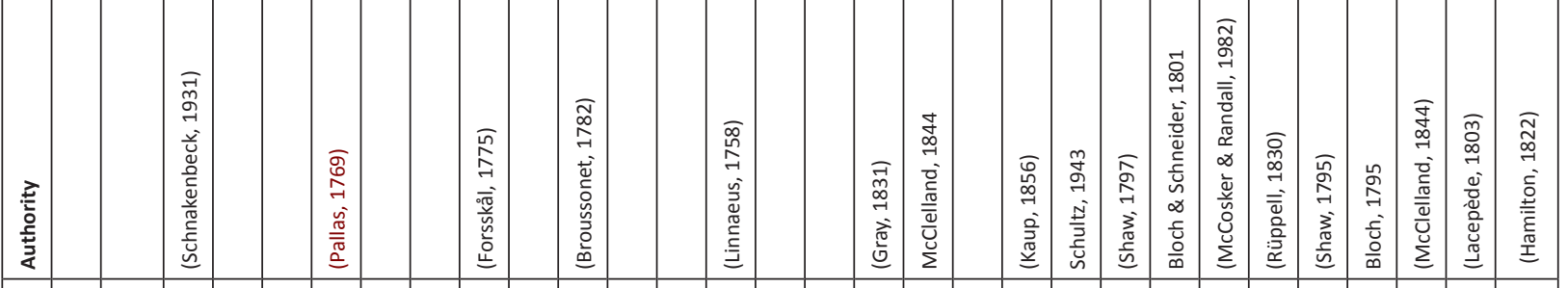

\begin{tabular}{|c|c|c|c|c|c|c|c|c|c|c|c|c|c|c|c|c|c|c|c|c|c|c|c|c|c|c|}
\hline 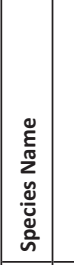 & & $\begin{array}{ll}3 \\
2\end{array}$ & & & 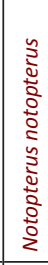 & & & 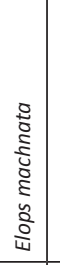 & & 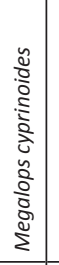 & & & 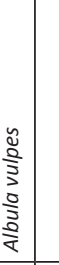 & & 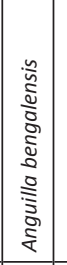 & 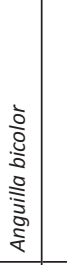 & 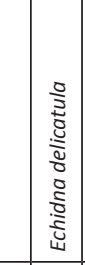 & 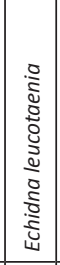 & 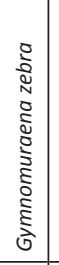 & 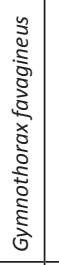 & 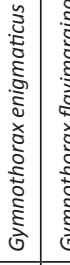 & & 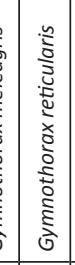 & 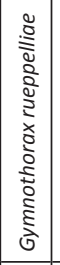 & 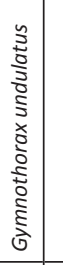 & 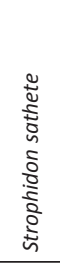 \\
\hline 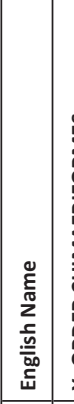 & 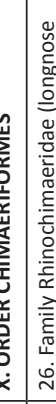 & 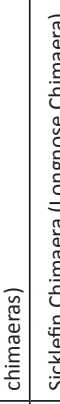 & 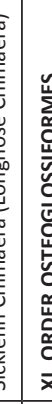 & 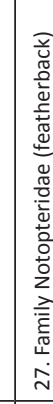 & 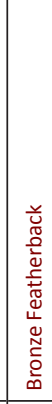 & 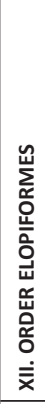 & 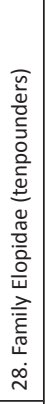 & 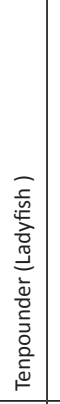 & 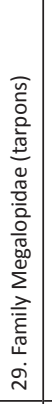 & 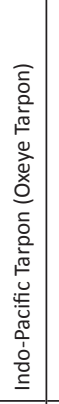 & 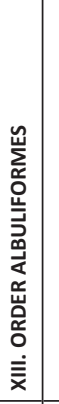 & 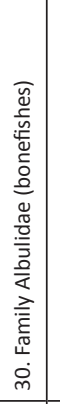 & 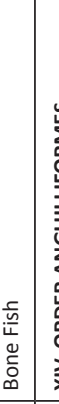 & 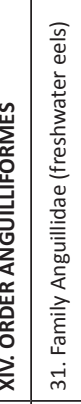 & 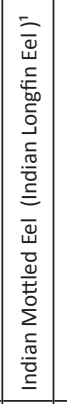 & 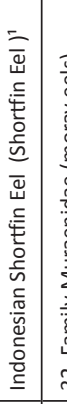 & 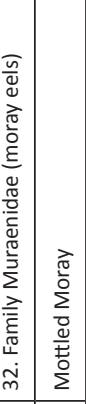 & 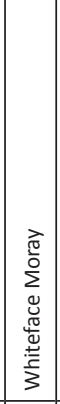 & 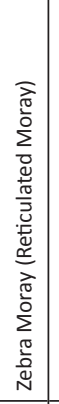 & 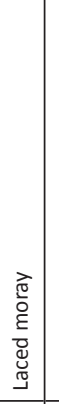 & 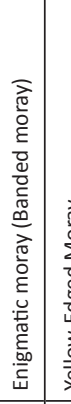 & 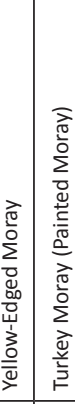 & 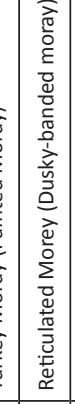 & 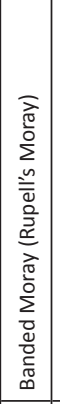 & 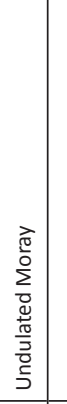 & 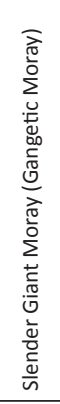 \\
\hline & & $\approx$ & 6 & & $\Phi$ & & & $\stackrel{\infty}{\infty}$ & & $\infty$ & & & $\hat{\infty}$ & & $\infty$ & $\infty$ & ஃ & $\vec{\sigma}$ & $\delta$ & ๓ & $\begin{array}{lll}\text { के } & \text {. }\end{array}$ & ఓ & ô & $\begin{array}{ll}\infty \\
\infty\end{array}$ & g & ঃ \\
\hline
\end{tabular}




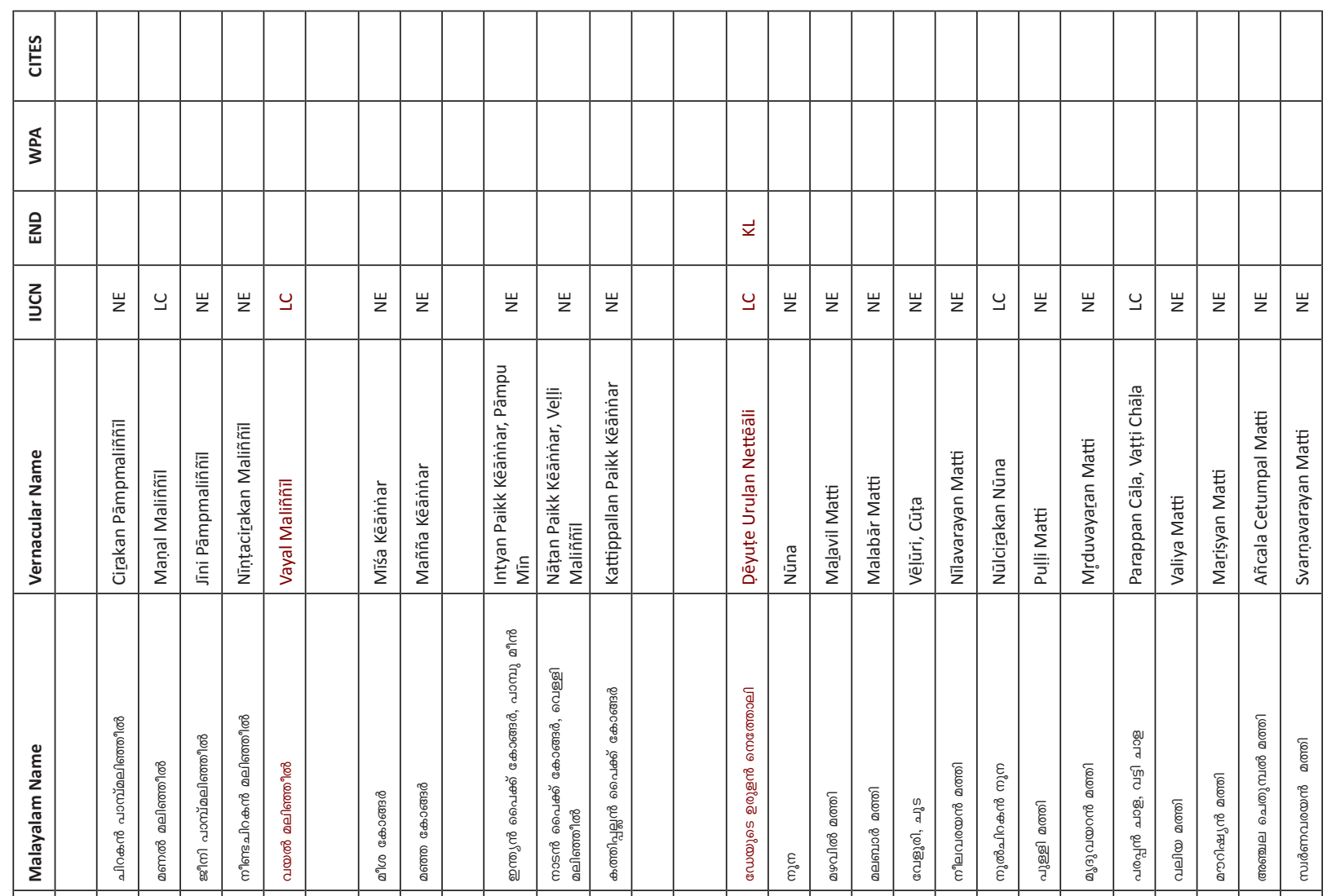

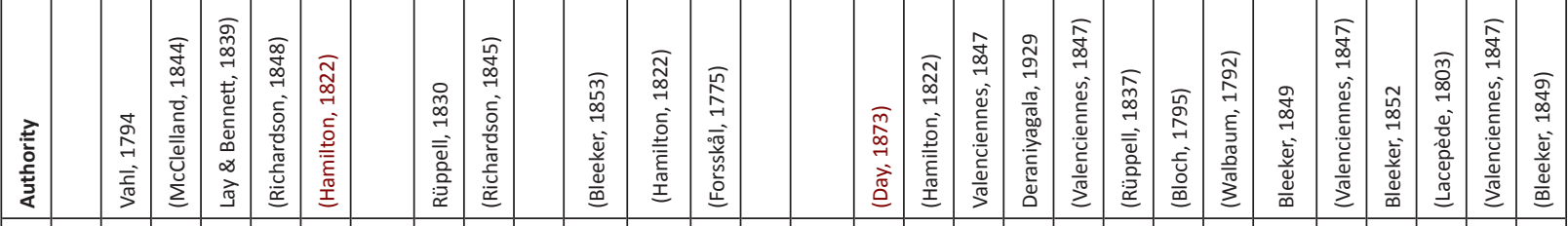

\begin{tabular}{|c|c|c|c|c|c|c|c|c|c|c|c|c|c|c|c|c|c|c|c|c|}
\hline 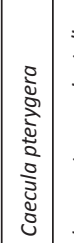 & 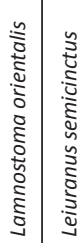 & 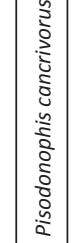 & 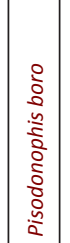 & 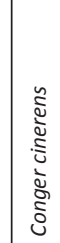 & 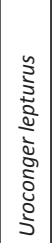 & 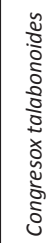 & 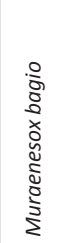 & 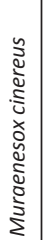 & 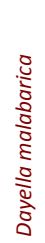 & 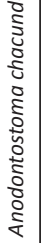 & & 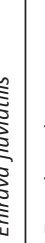 & 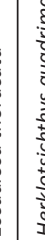 & 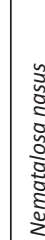 & 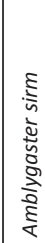 & 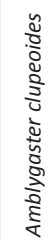 & 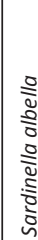 & & & 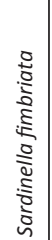 \\
\hline
\end{tabular}

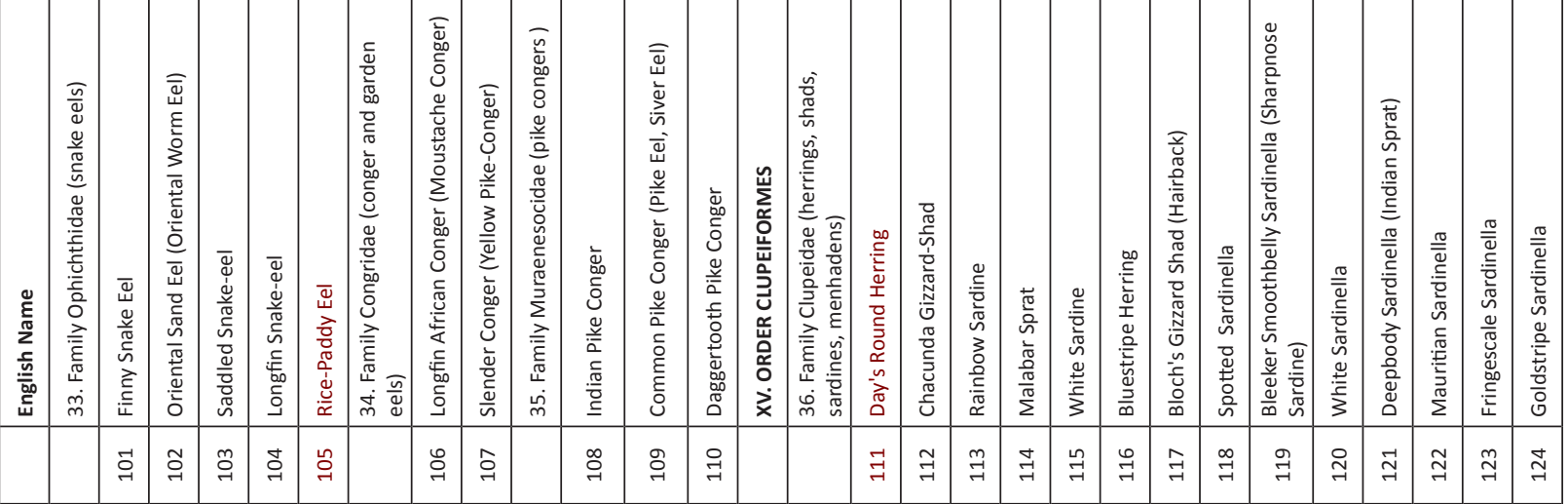




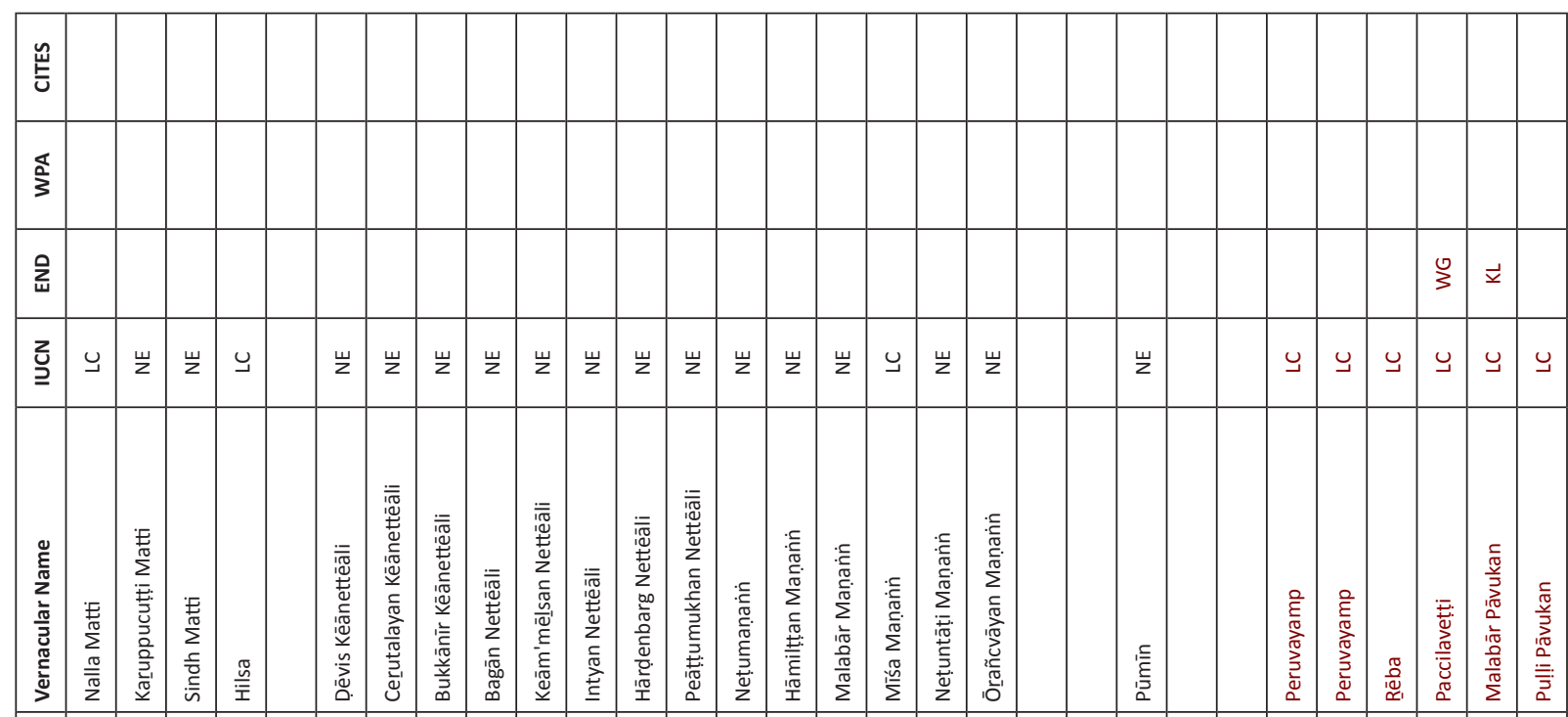

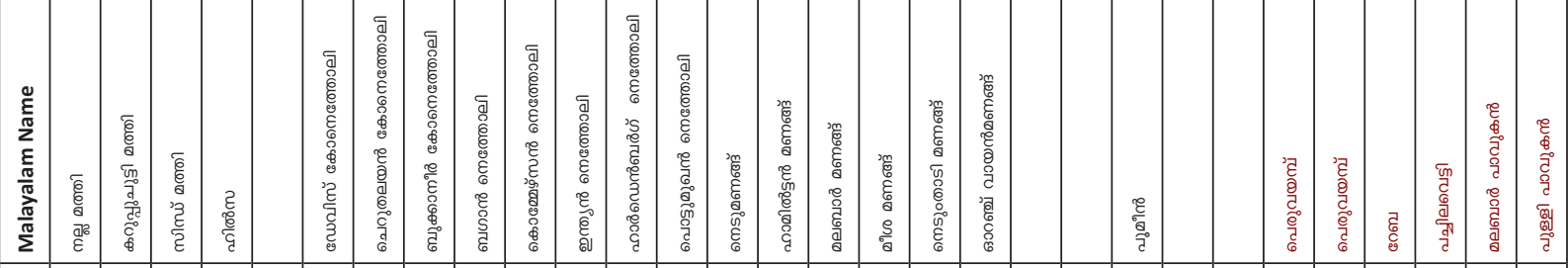

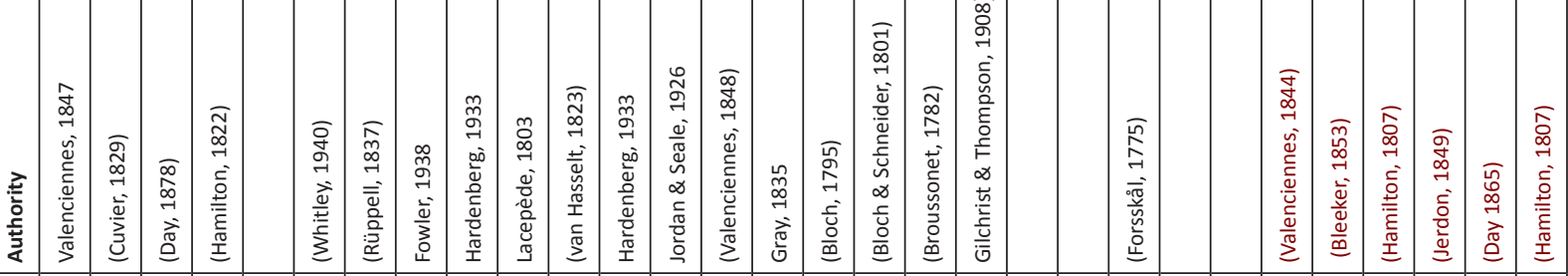

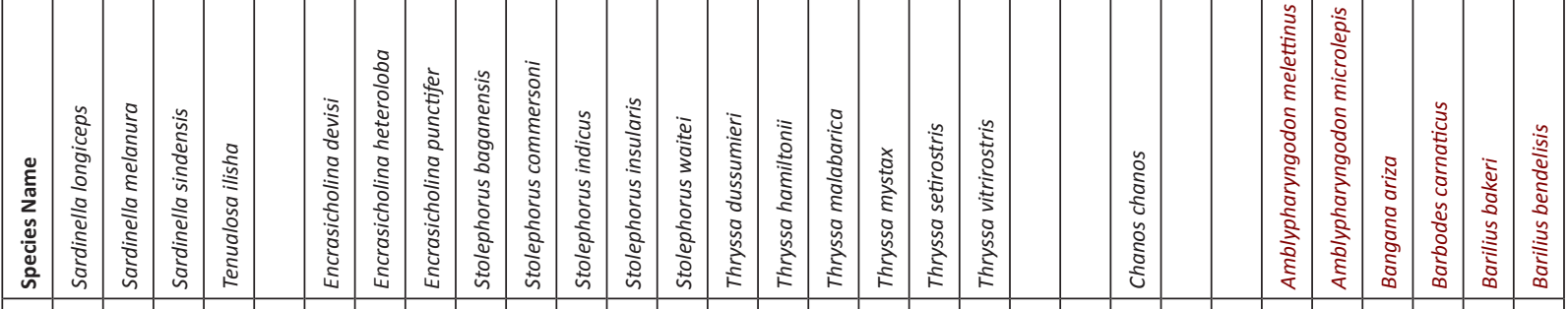

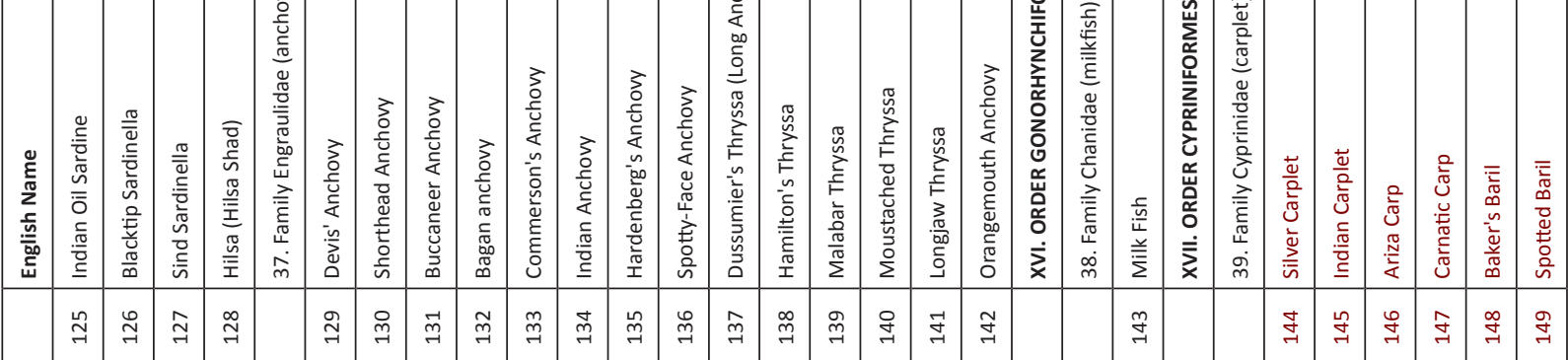




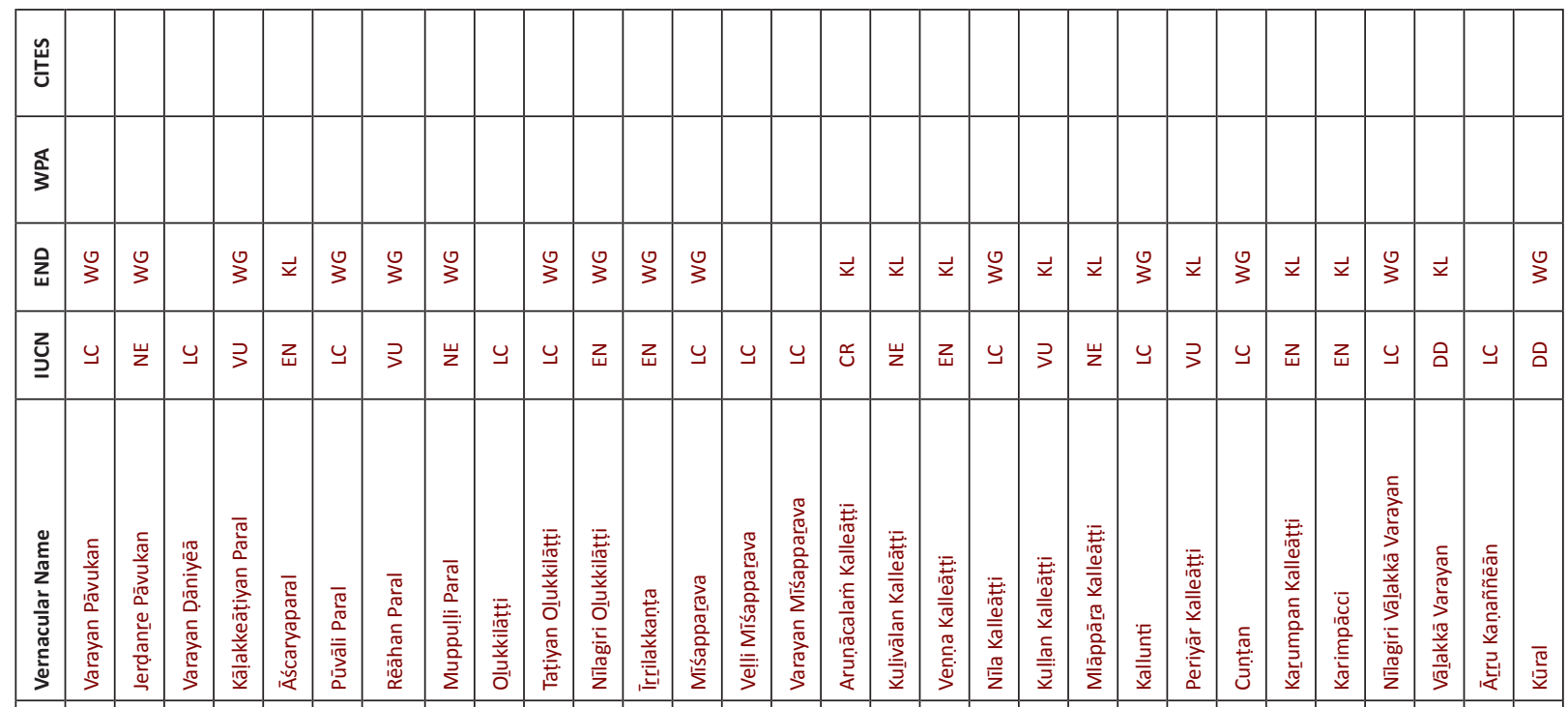

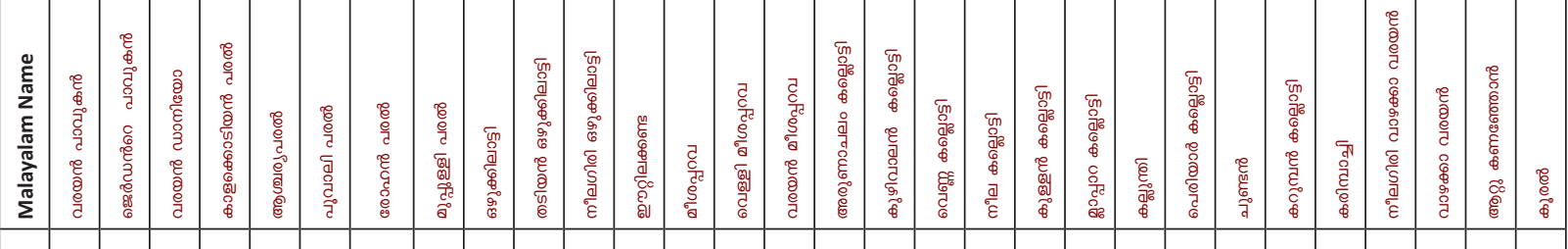

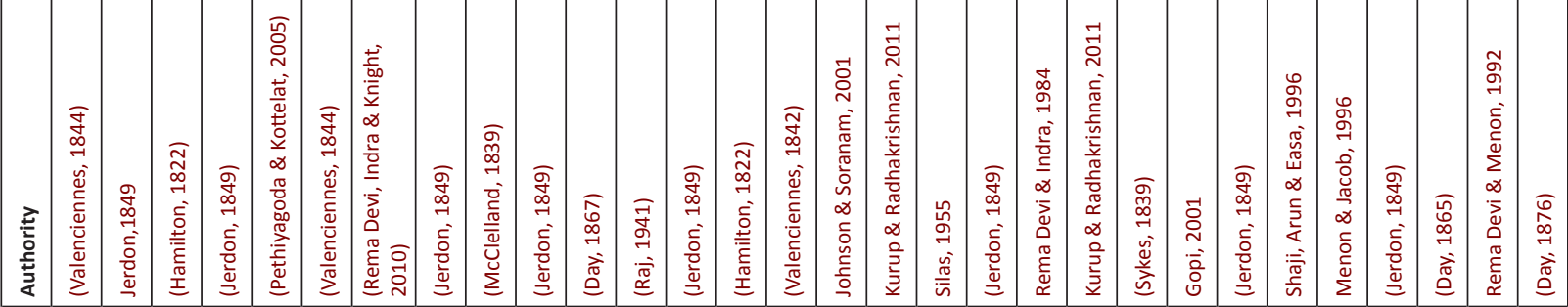

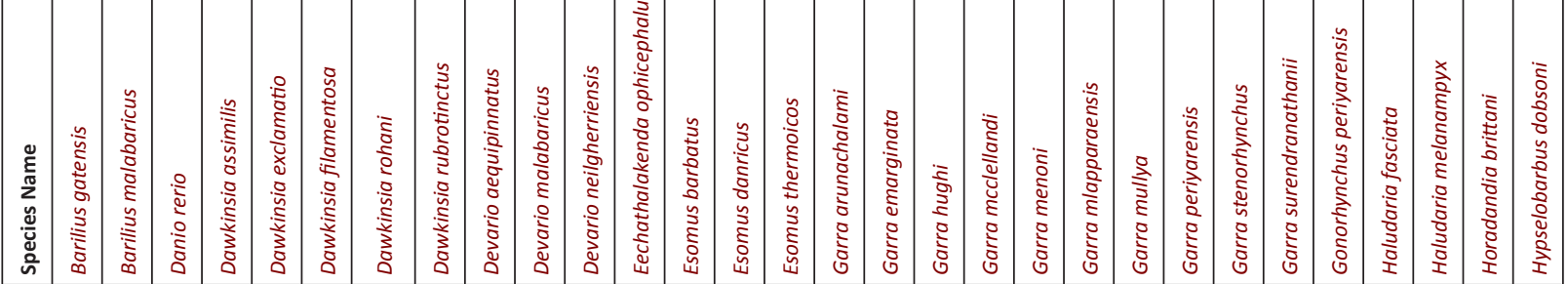

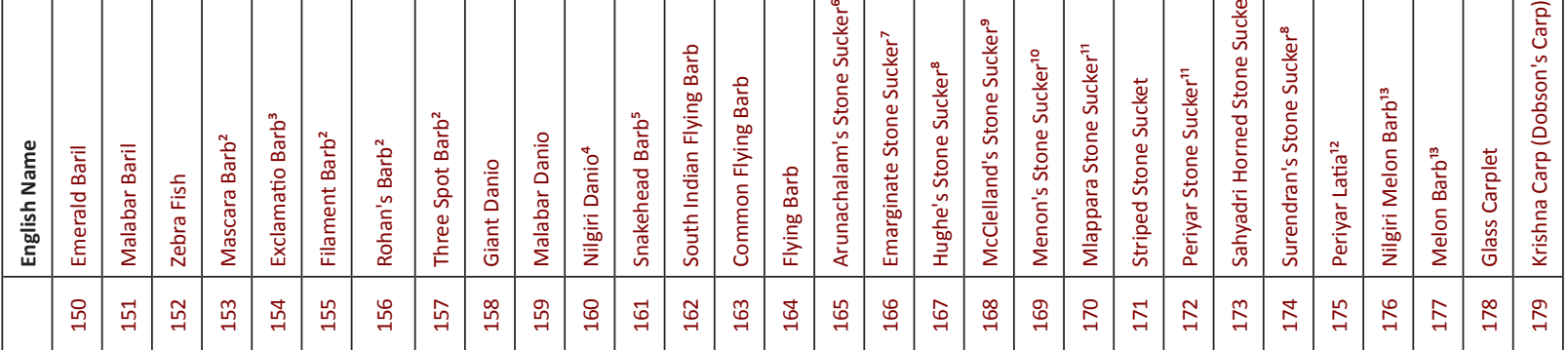




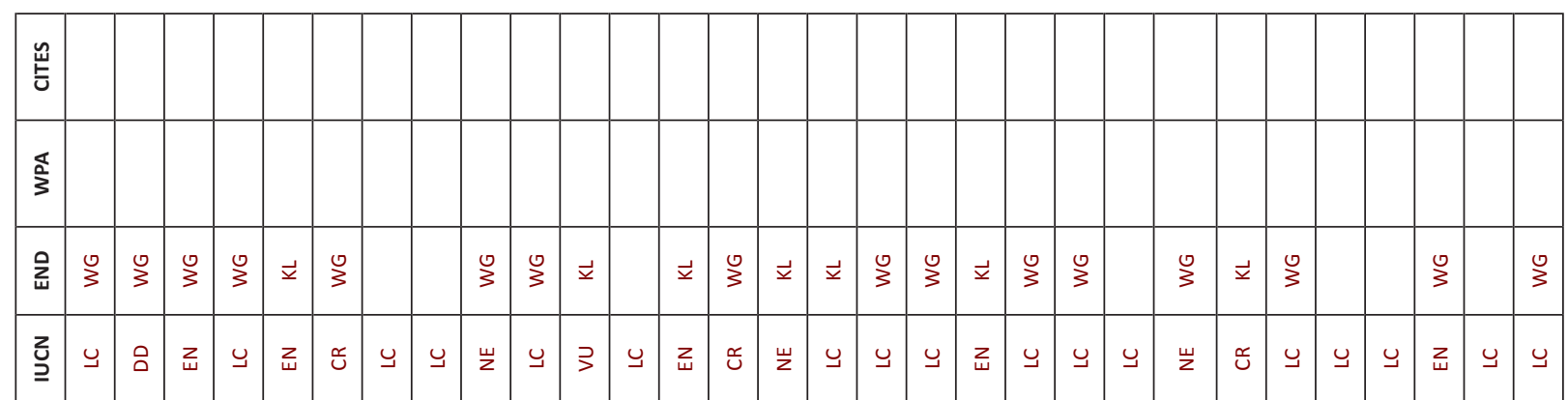

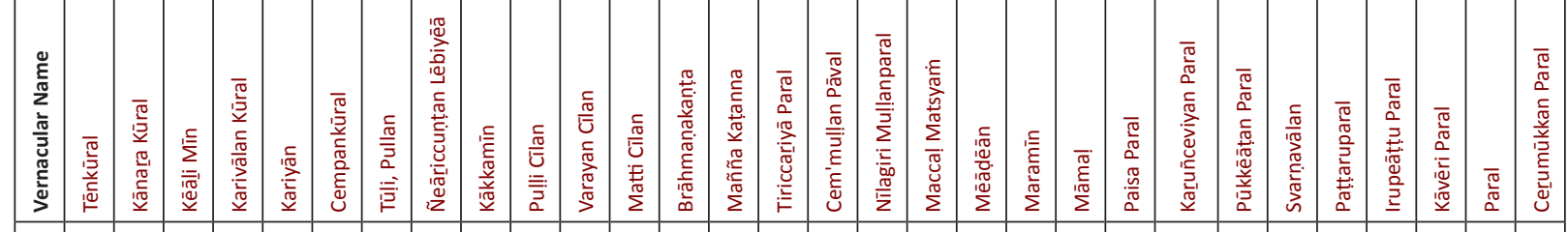

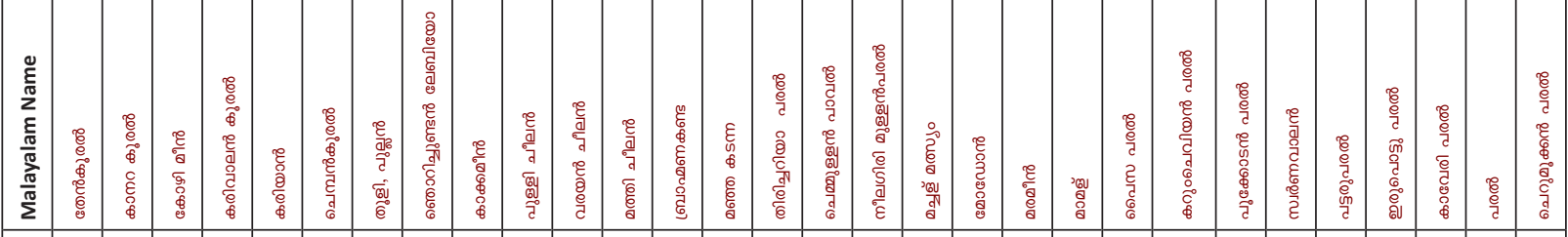

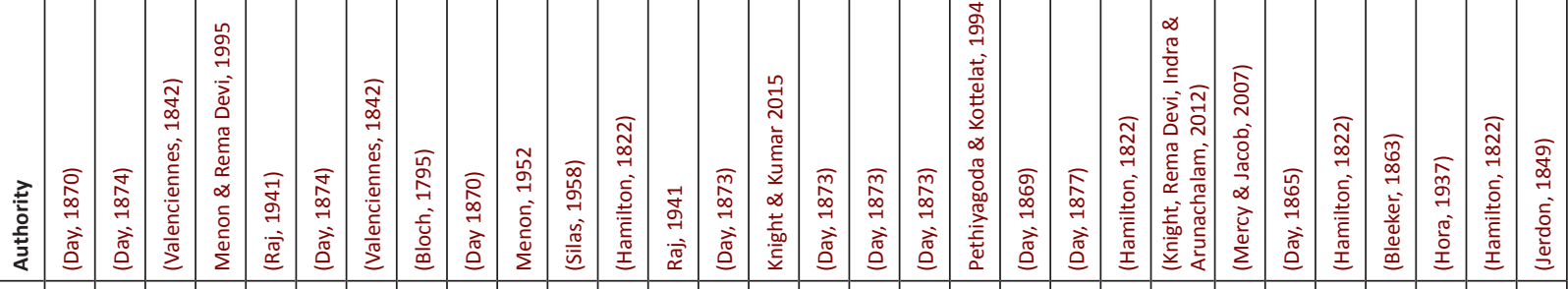

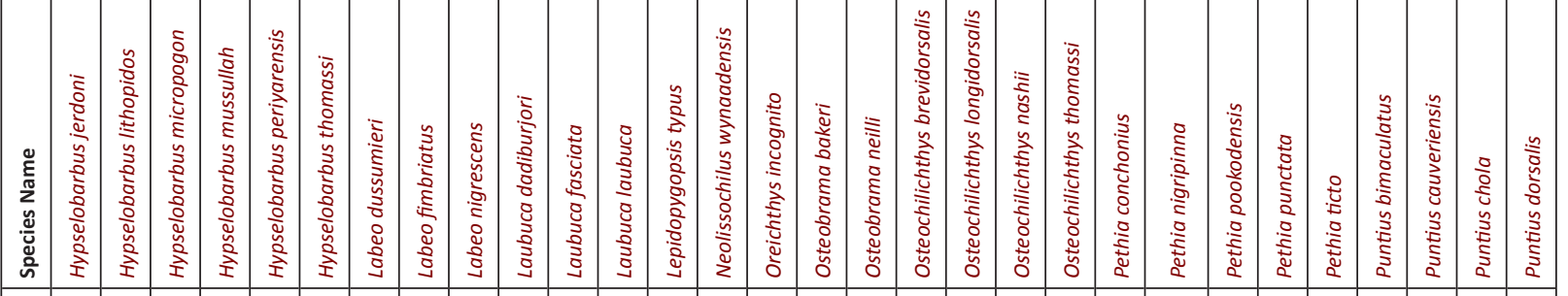

\begin{tabular}{|c|c|c|c|c|c|c|c|c|c|c|c|c|c|c|c|c|c|c|c|c|c|c|c|c|c|}
\hline 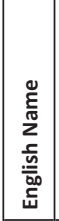 & 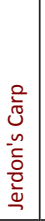 & 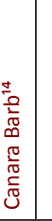 & & 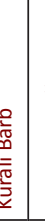 & 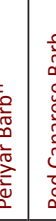 & 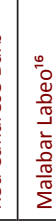 & 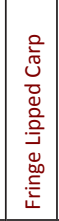 & $\begin{array}{l} \\
\frac{8}{0} \\
\frac{0}{0} \\
\frac{0}{0} \\
\frac{0}{0} \\
\end{array}$ & 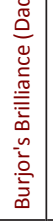 & 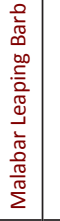 & 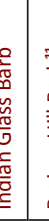 & 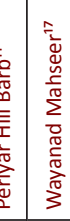 & 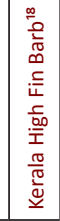 & 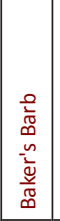 & 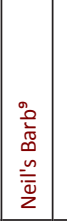 & 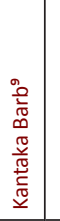 & 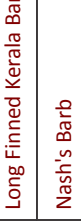 & 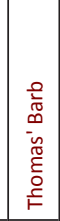 & 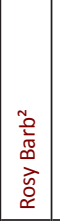 & 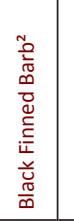 & & 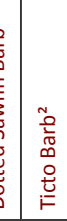 & 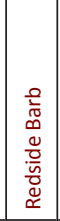 & 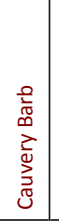 & 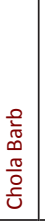 \\
\hline & $\underset{\sim}{\infty}$ & $\overrightarrow{\underline{\sigma}}$ & & $\infty_{-\infty}^{\infty}$ & 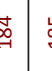 & $\underset{ل ٍ ~}{\circ}$ & $\stackrel{\infty}{\rightarrow}$ & 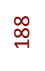 & $\underset{\sim}{\mathbb{D}}$ & $\stackrel{8}{9}$ & ने & $\stackrel{\Omega}{\Omega}$ & $\stackrel{t}{g}$ & $\stackrel{\text { 号 }}{\sigma}$ & $\stackrel{8}{\circ}$ & a & 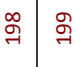 & 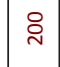 & ثे & ป̃ & $\stackrel{m}{\sim}$ & $\stackrel{\text { D }}{\sim}$ & $\stackrel{\text { L }}{\text { N }}$ & $\hat{i}$ & $\stackrel{\text { : }}{\sim}$ \\
\hline
\end{tabular}




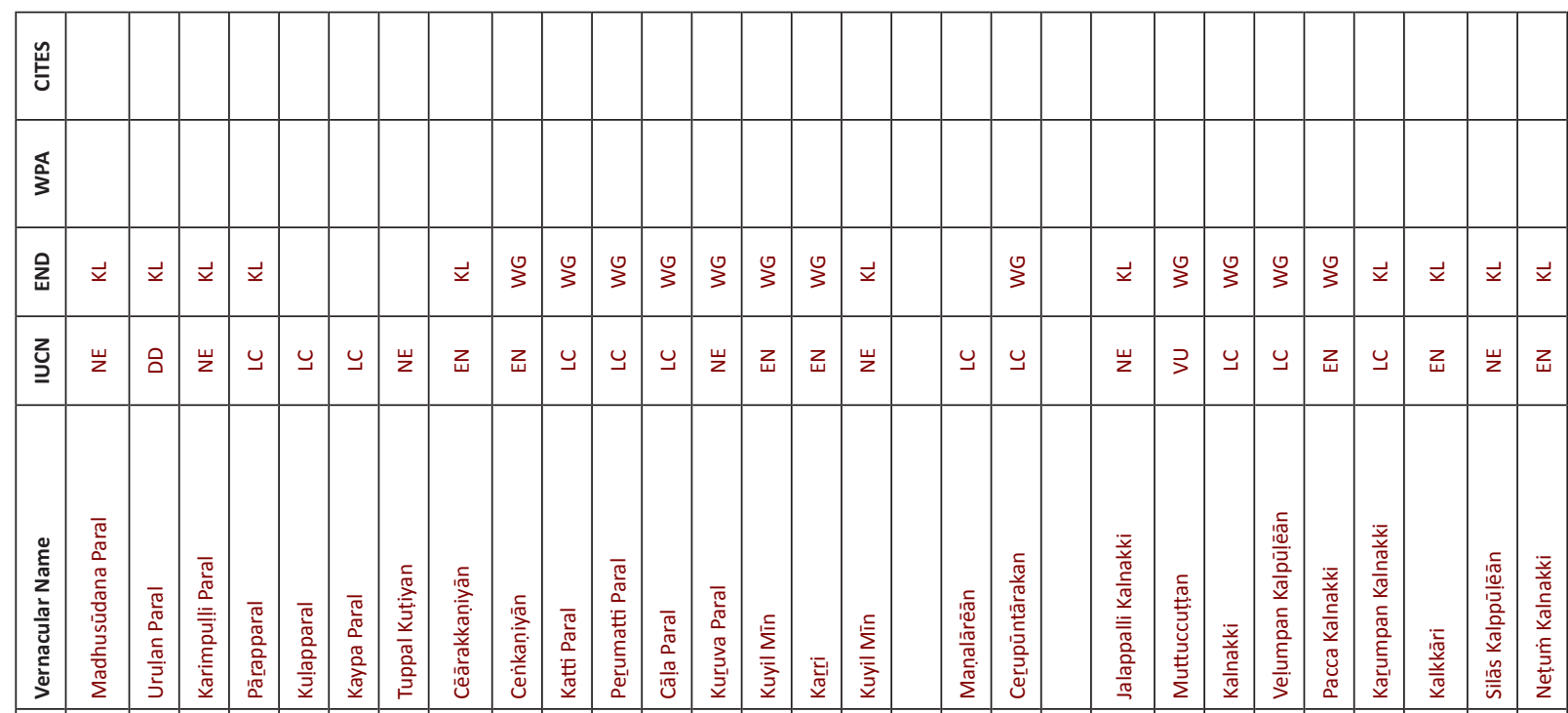

\begin{tabular}{|c|c|c|c|c|c|c|c|c|c|c|c|c|c|c|c|c|c|c|c|c|c|c|c|c|}
\hline & & 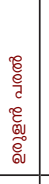 & 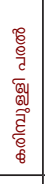 & 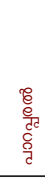 & 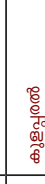 & 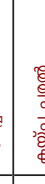 & 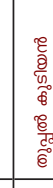 & 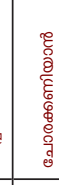 & 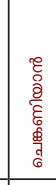 & 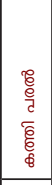 & 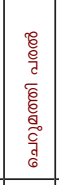 & 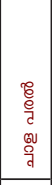 & 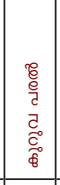 & 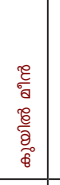 & 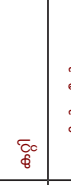 & 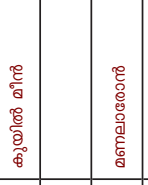 & 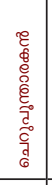 & 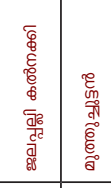 & \begin{tabular}{|c|}
$\bar{\Phi}$ \\
$\bar{\Phi}$ \\
$\tilde{\sigma}$ \\
$\bar{\phi}$ \\
\end{tabular} & 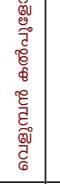 & 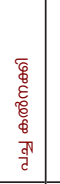 & & & 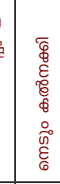 \\
\hline & 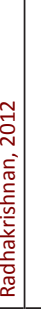 & 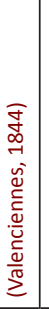 & 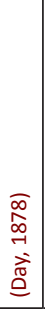 & 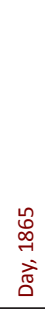 & 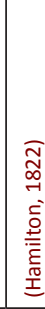 & $\begin{array}{l}0 \\
0 \\
0 \\
0 \\
\vdots \\
0 \\
0\end{array}$ & 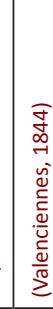 & 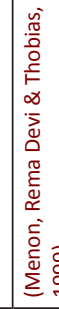 & 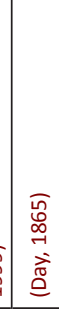 & 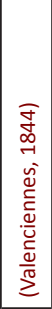 & 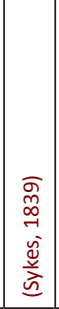 & 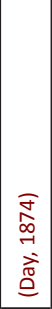 & 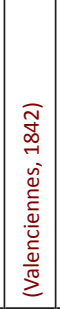 & 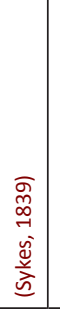 & 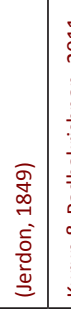 & 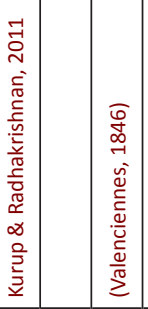 & 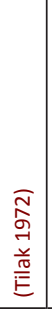 & 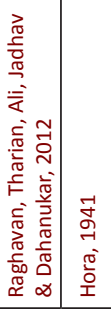 & 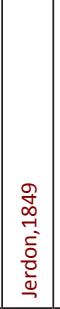 & 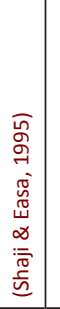 & 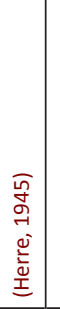 & 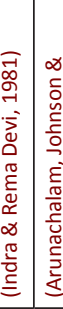 & 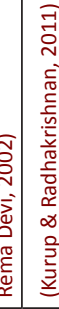 & 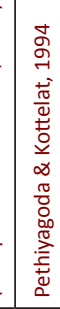 \\
\hline
\end{tabular}

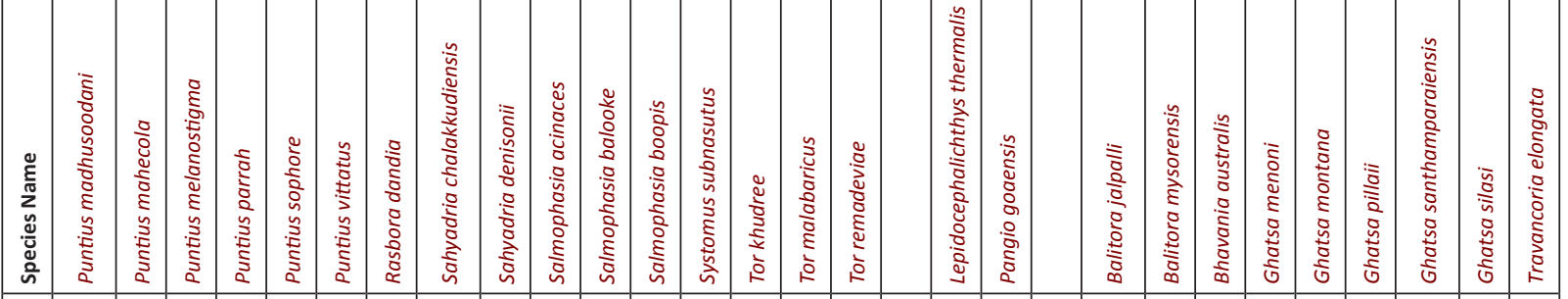




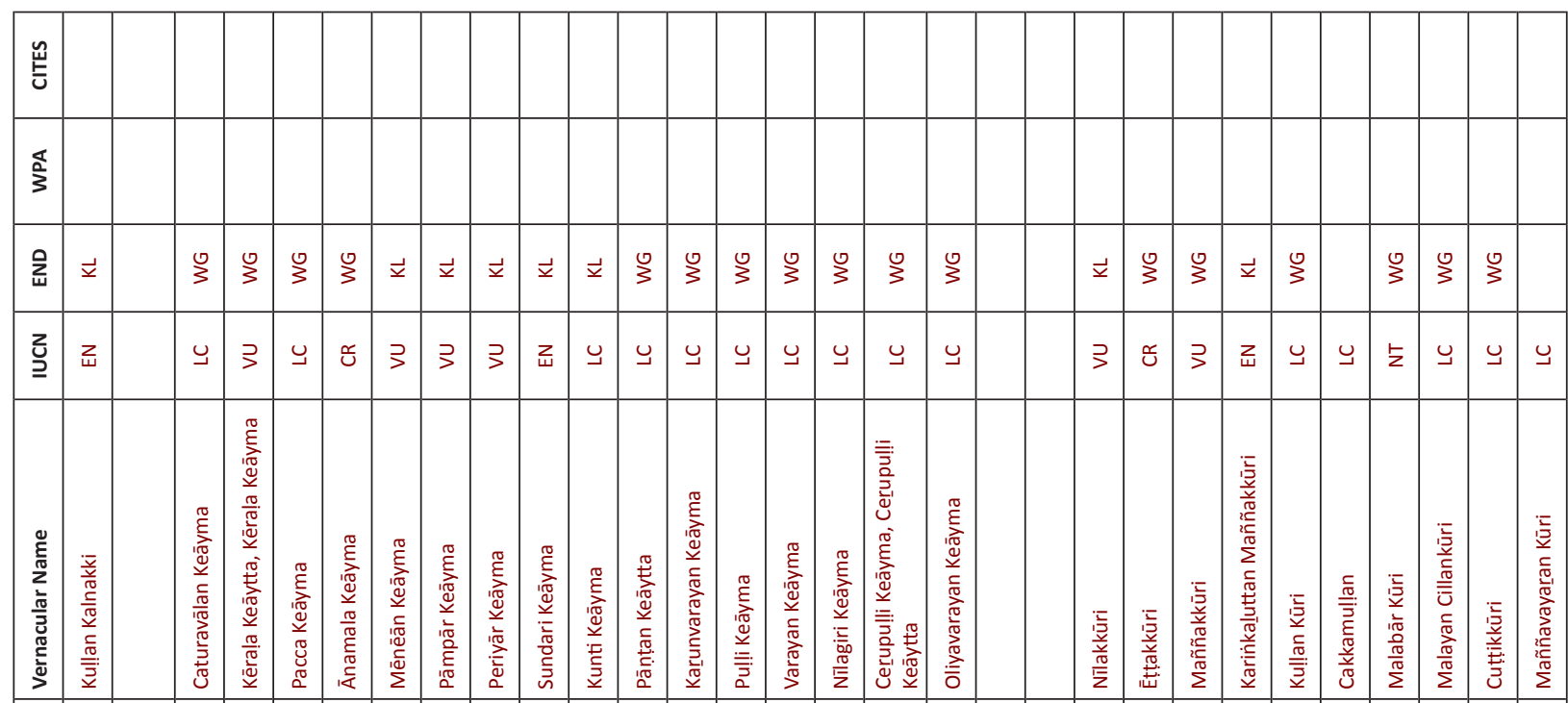

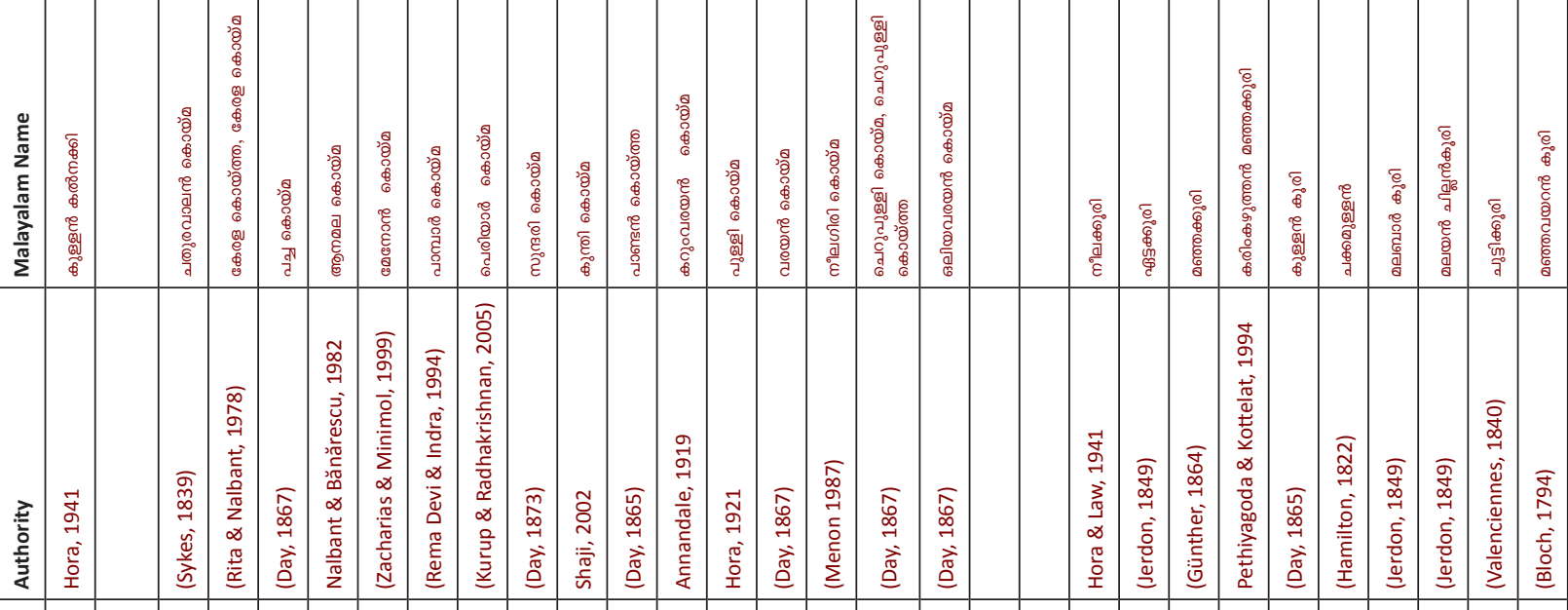

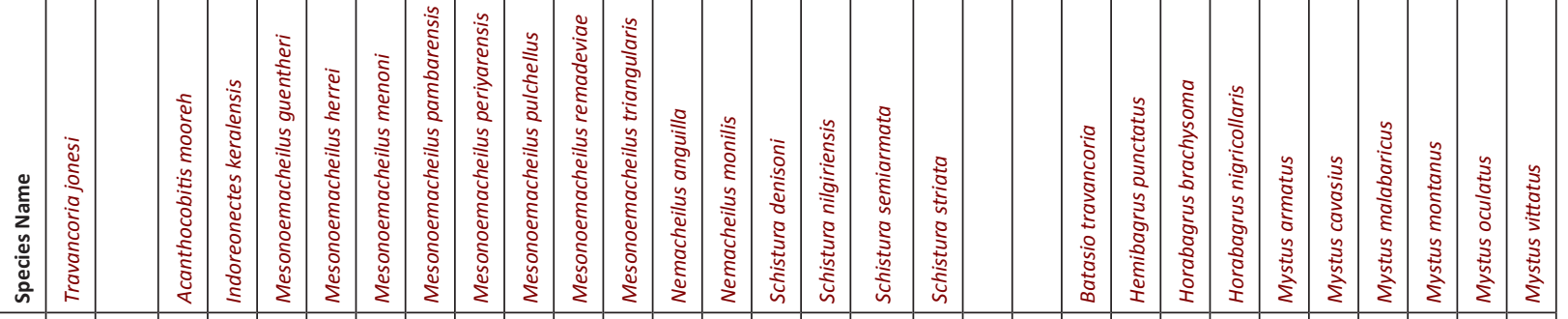

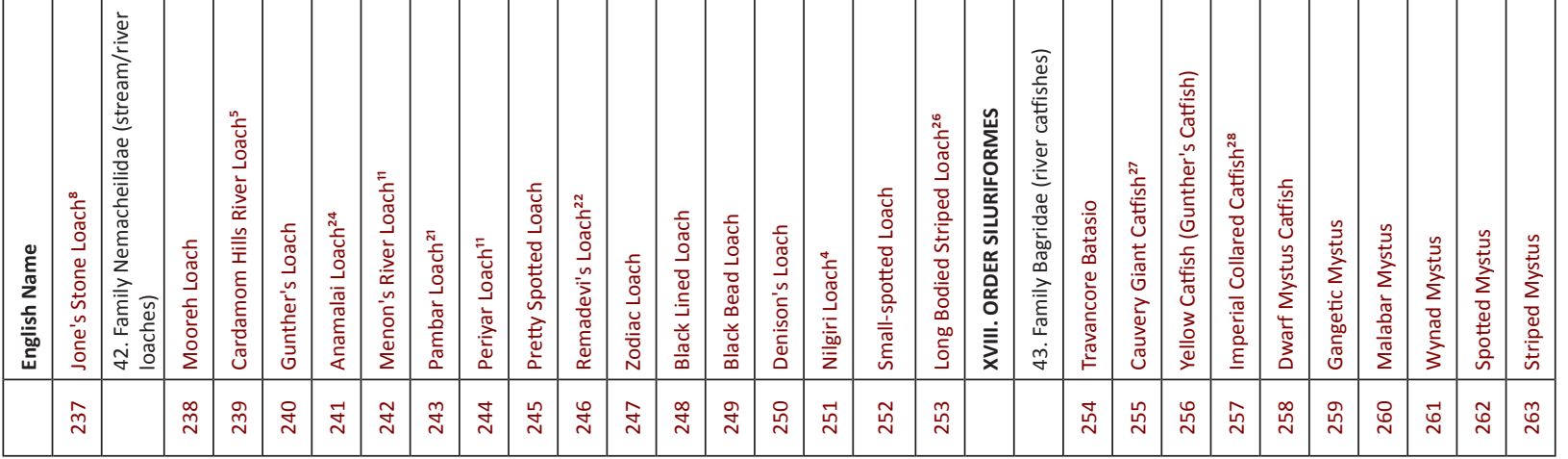




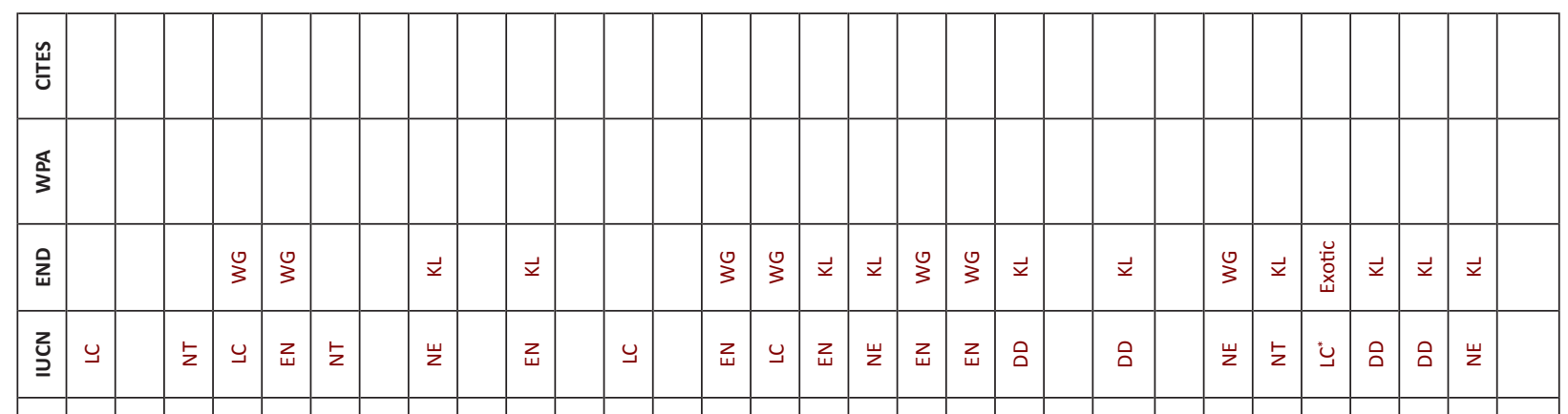

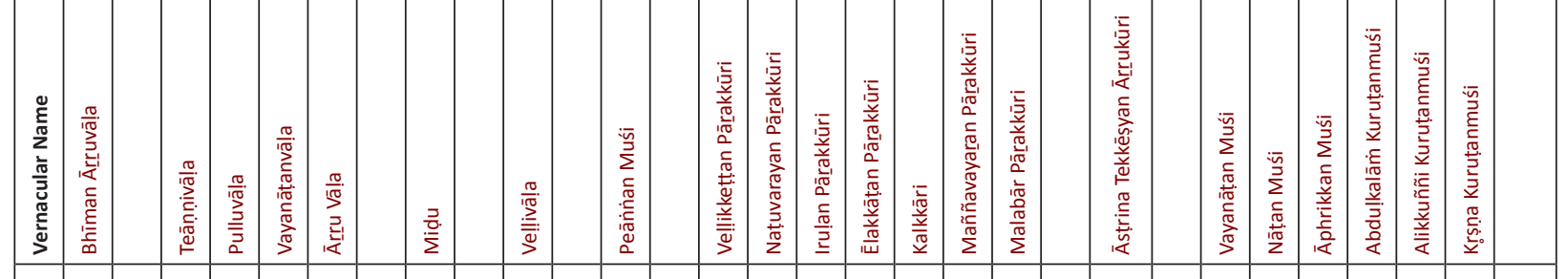

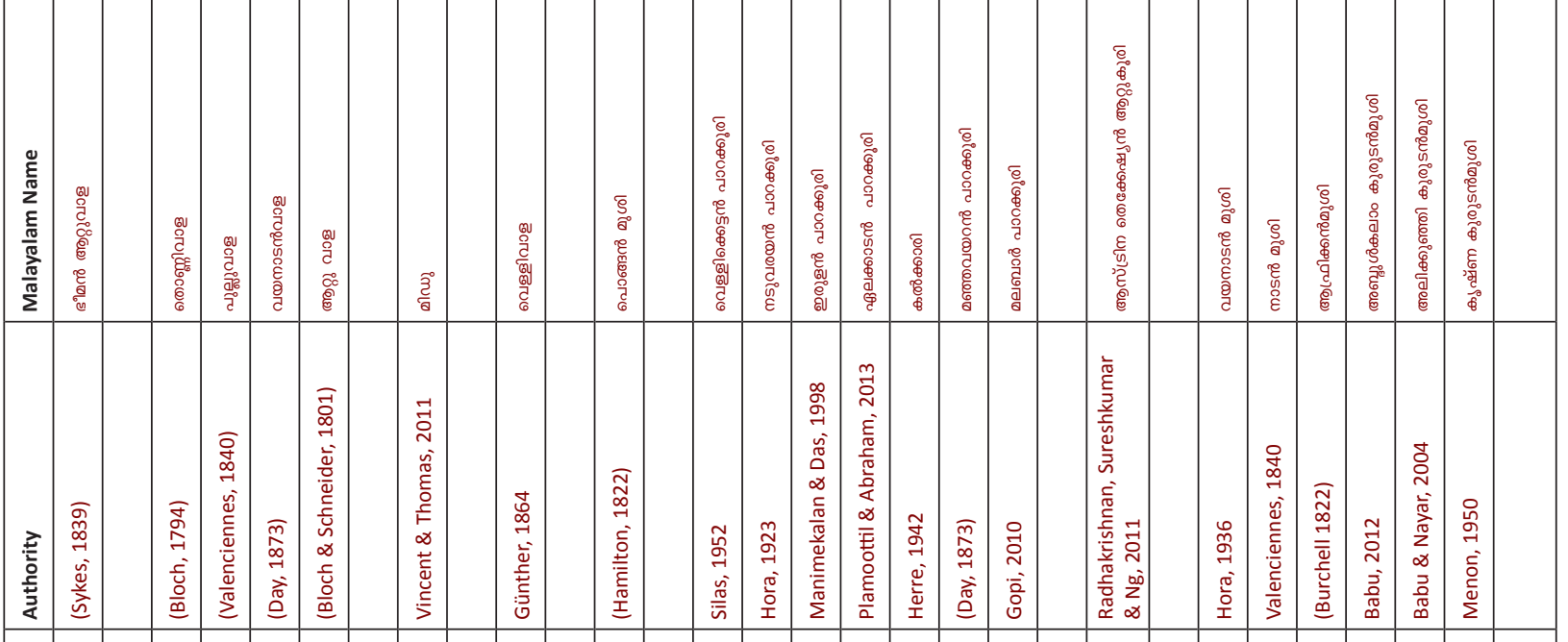

\begin{tabular}{|c|c|c|c|c|c|c|c|c|c|c|c|c|c|c|c|c|c|c|c|c|}
\hline 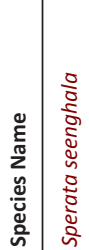 & 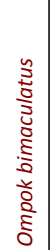 & 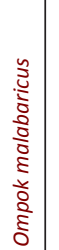 & 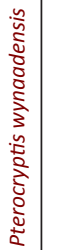 & 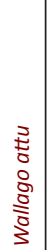 & 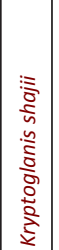 & 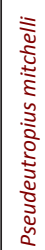 & 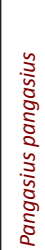 & 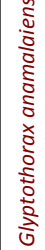 & 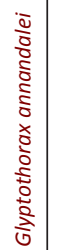 & 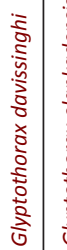 & 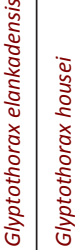 & $\left|\begin{array}{r}0 \\
0 \\
0 \\
0 \\
0 \\
0 \\
0 \\
g \\
0 \\
0 \\
0 \\
0 \\
0 \\
0 \\
0 \\
0 \\
0 \\
0\end{array}\right|$ & 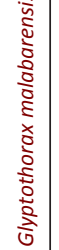 & 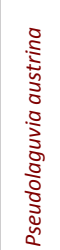 & $\begin{array}{l}\overline{\bar{g}} \\
.\end{array}$ & 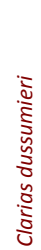 & 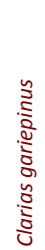 & 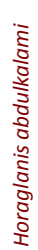 & & 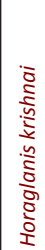 \\
\hline
\end{tabular}

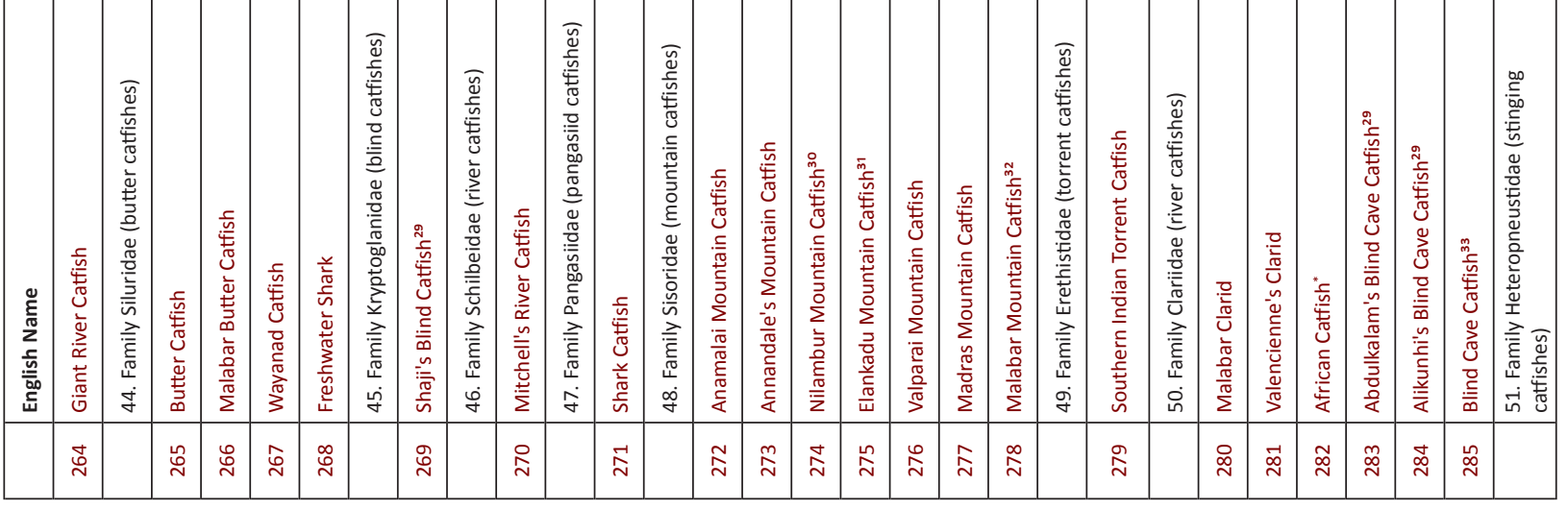




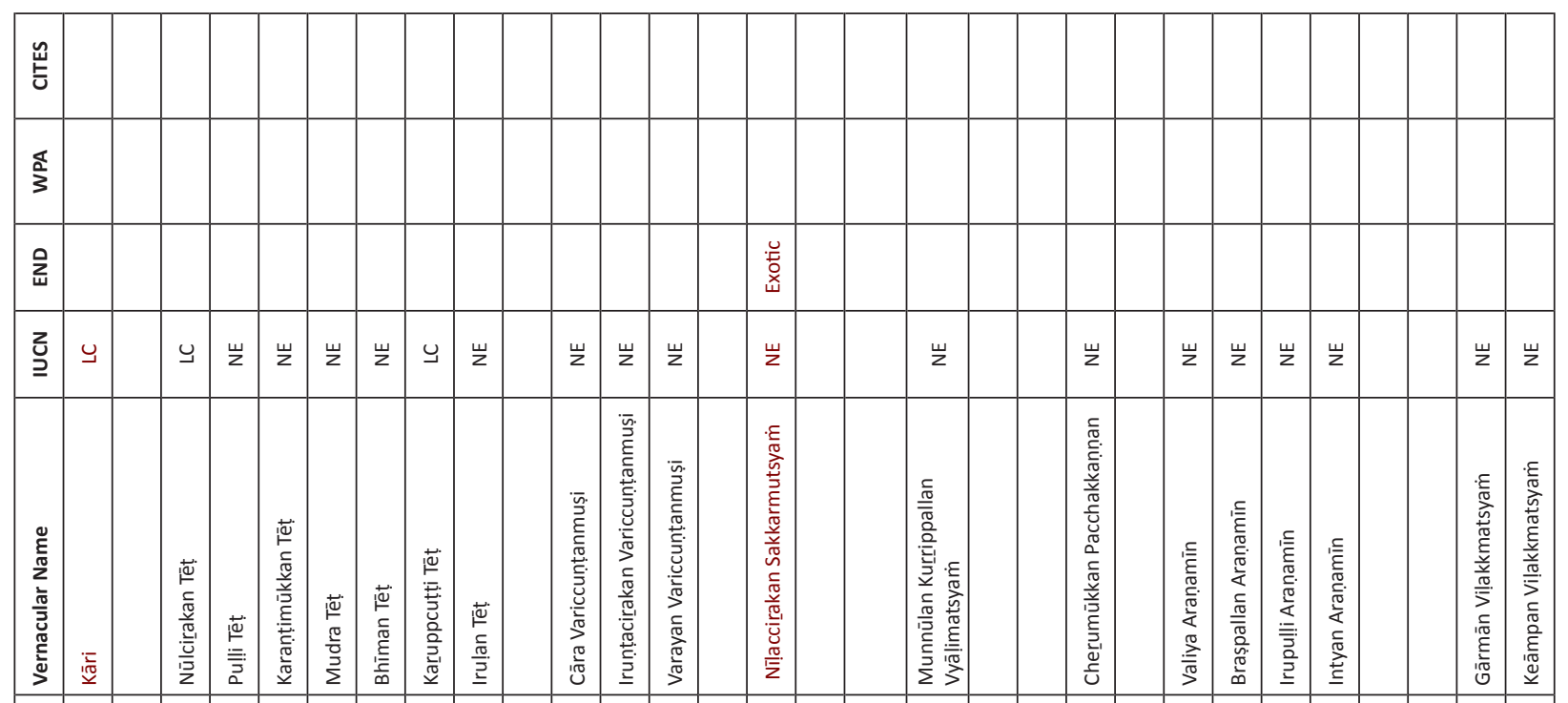

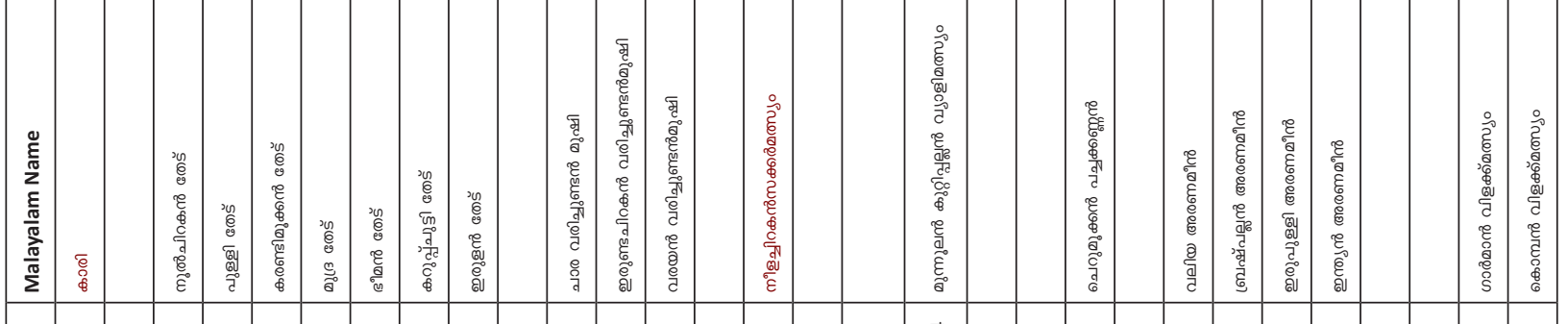

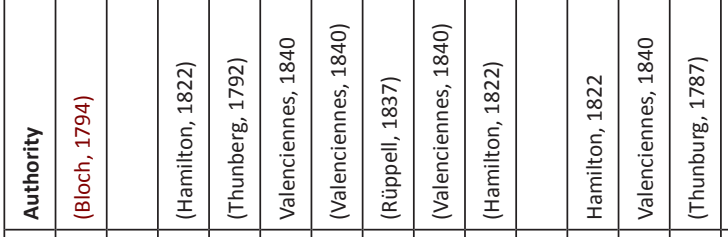

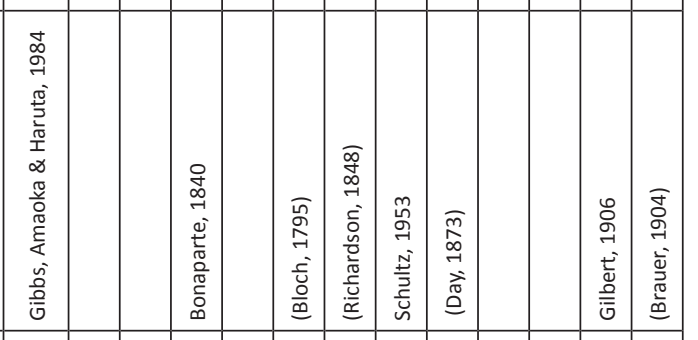

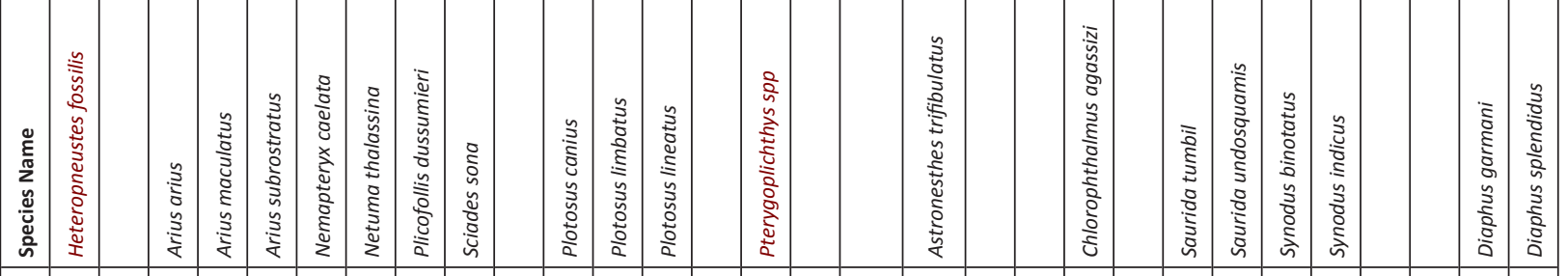

\begin{tabular}{|c|c|c|c|c|c|c|c|c|c|c|c|c|c|c|c|c|c|c|c|c|c|c|c|}
\hline 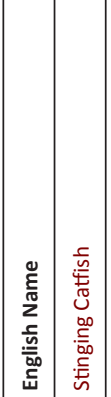 & 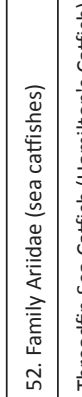 & 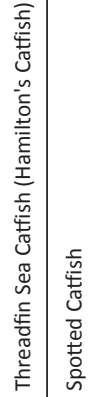 & 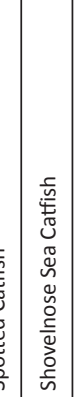 & 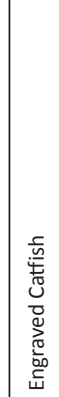 & 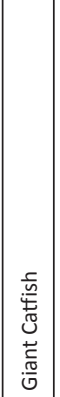 & 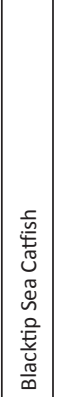 & 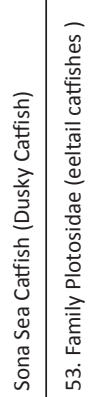 & 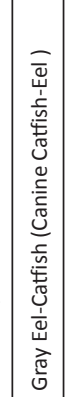 & 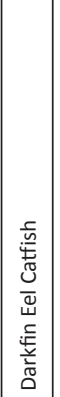 & 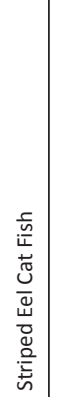 & 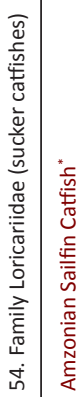 & 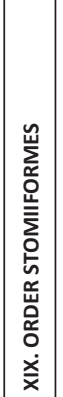 & 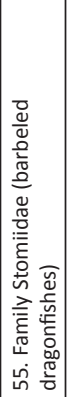 & 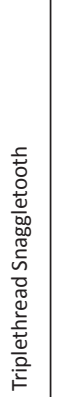 & 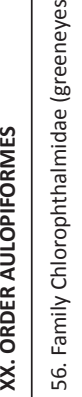 & 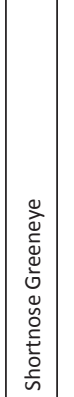 & 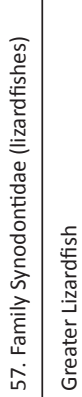 & 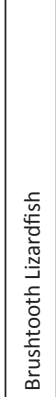 & 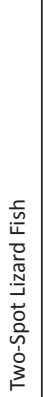 & & 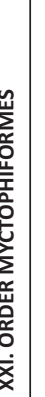 & & \\
\hline$\stackrel{\mathscr{D}}{\sim}$ & & \begin{tabular}{c|c}
$\stackrel{\infty}{\sim}$ & $\stackrel{\infty}{\sim}$
\end{tabular} & \begin{tabular}{c|c}
$:$ \\
$\stackrel{\sim}{\sim}$
\end{tabular} & ঃ & $\vec{N}$ & ন̃ & $\stackrel{\cap}{\sim}$ & 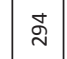 & $\stackrel{\stackrel{\sim}{\sim}}{\sim}$ & 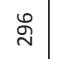 & & & & $\stackrel{\infty}{\sim}$ & & 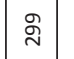 & $m$ & $\vec{m}$ & ح & & & & \\
\hline
\end{tabular}




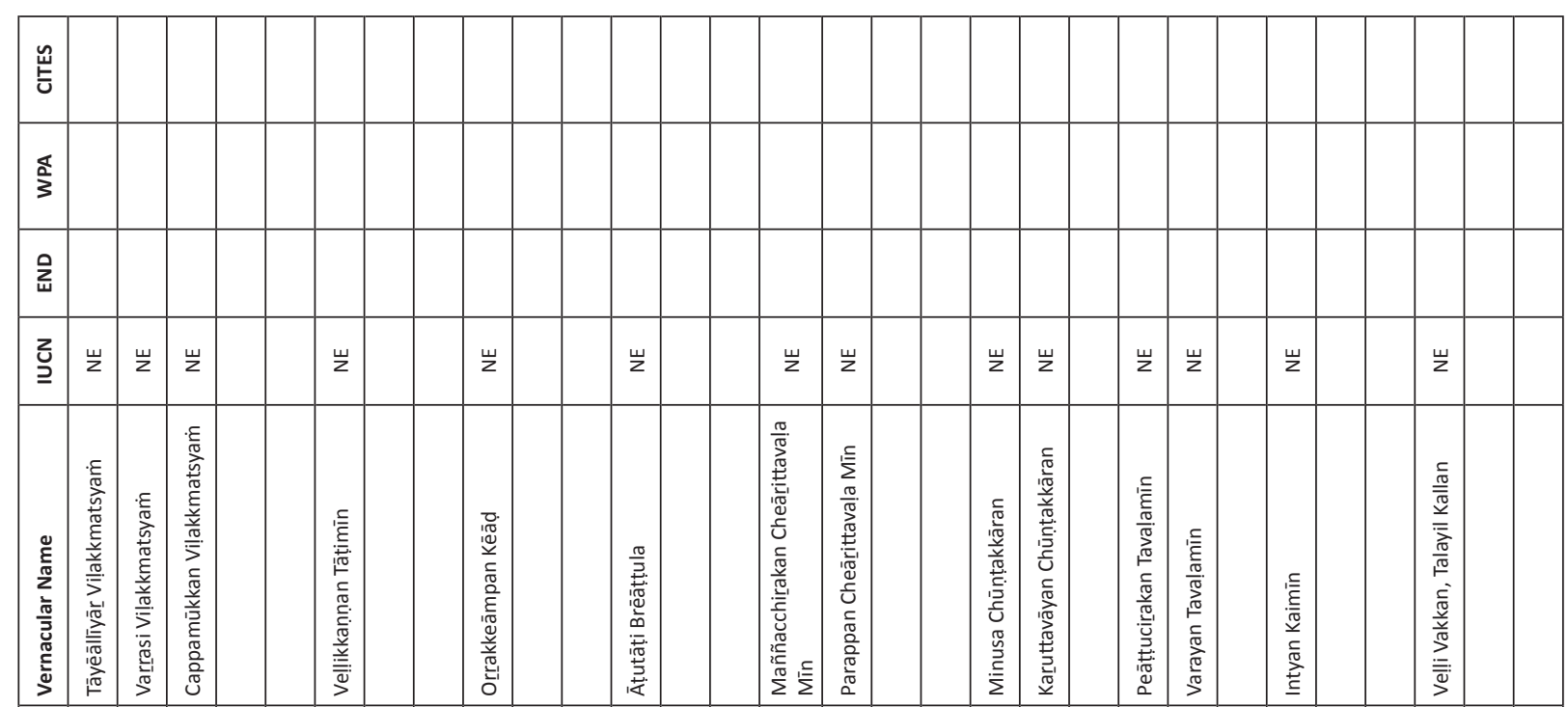

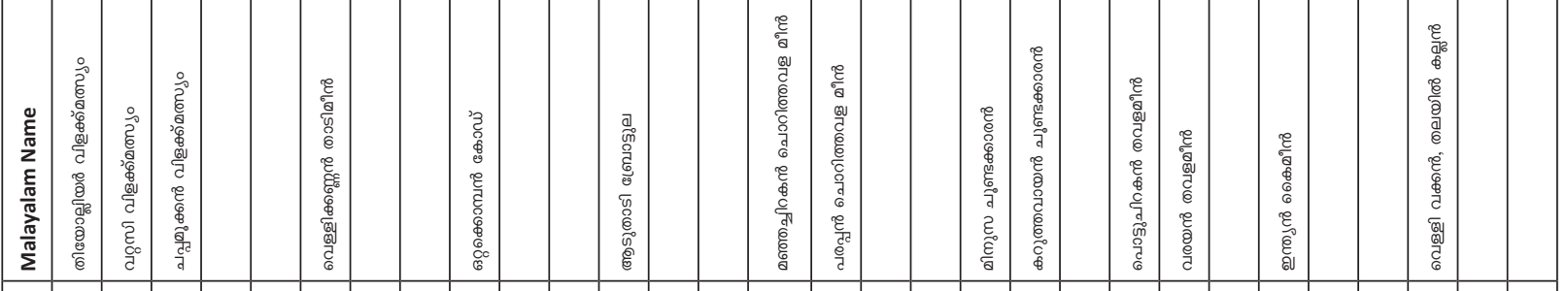

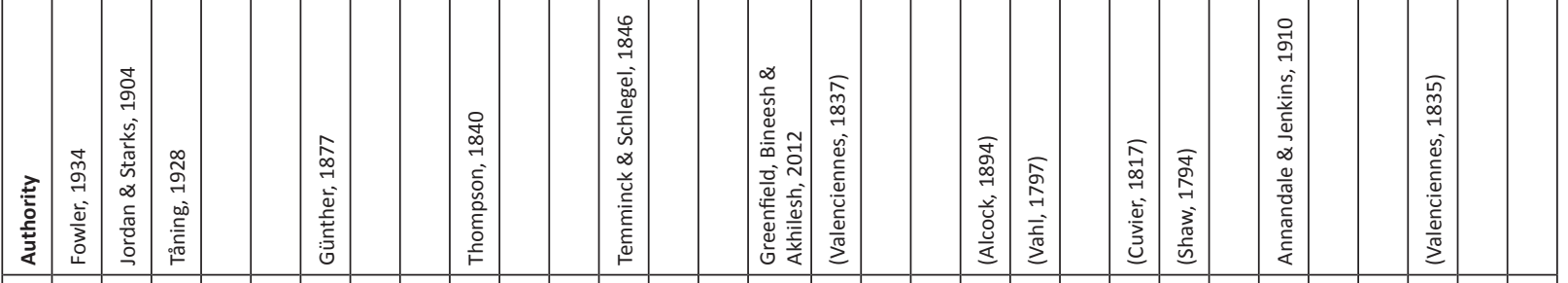

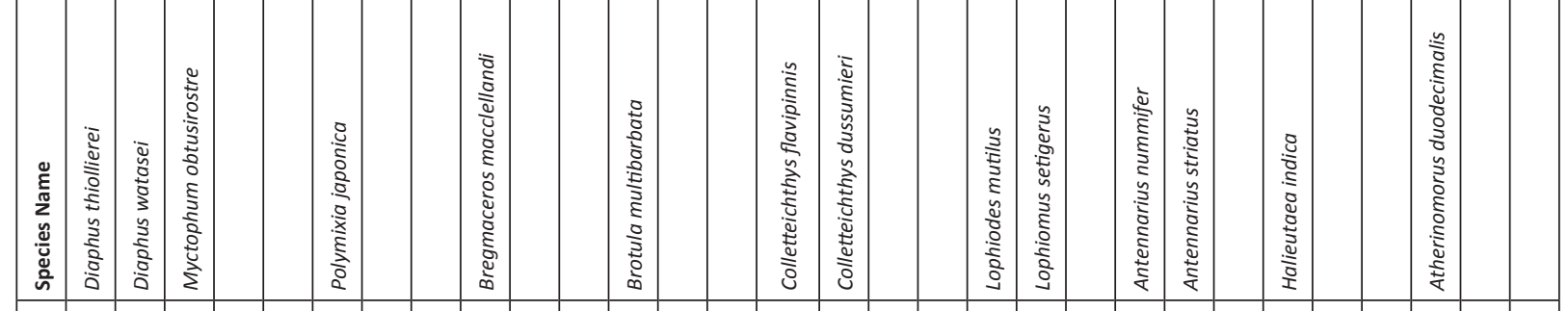

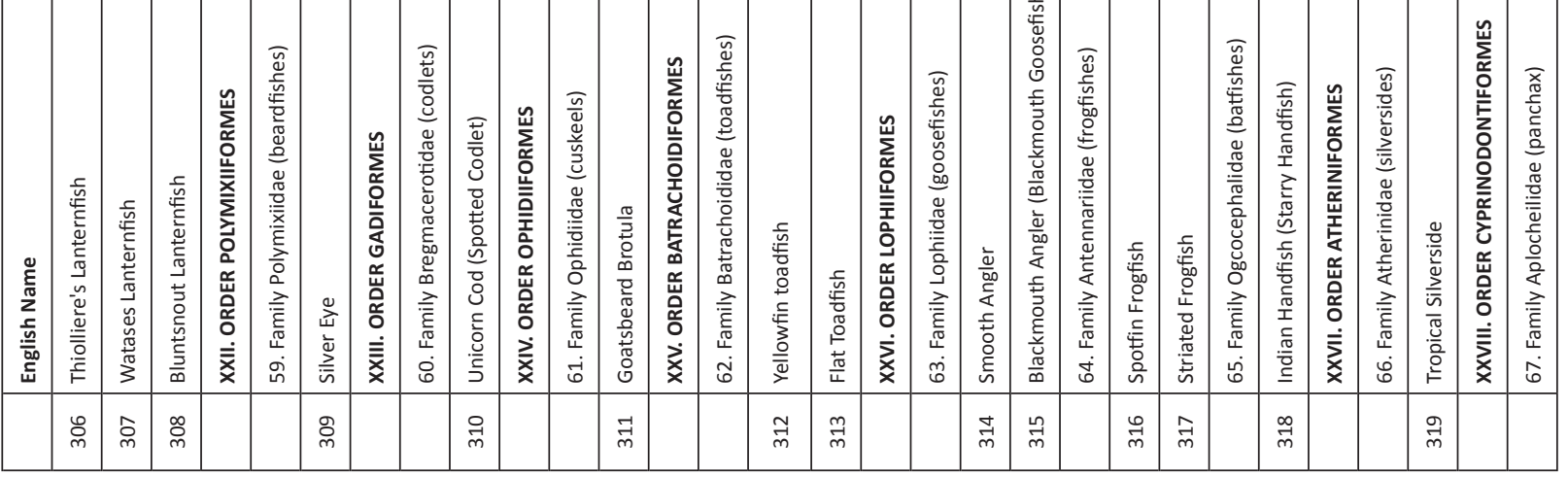




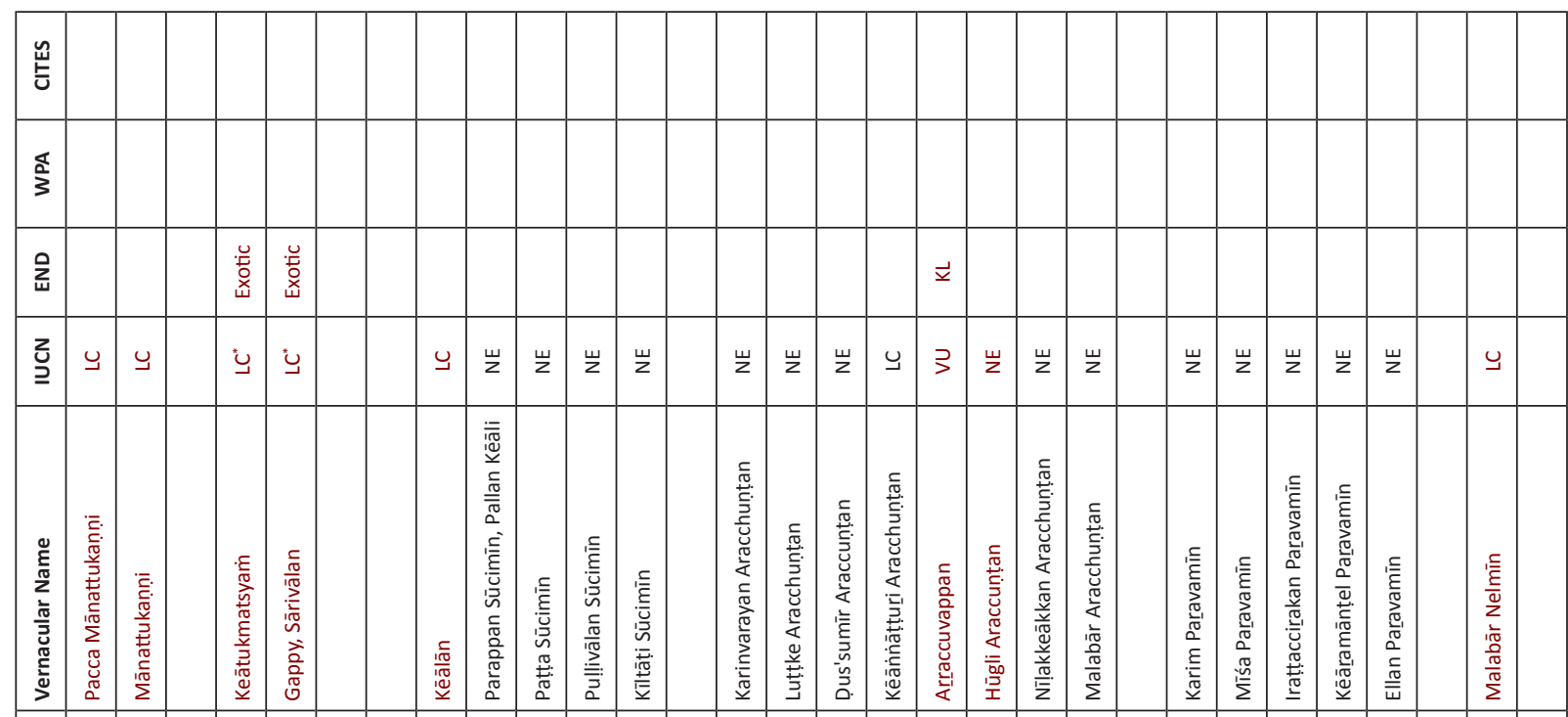

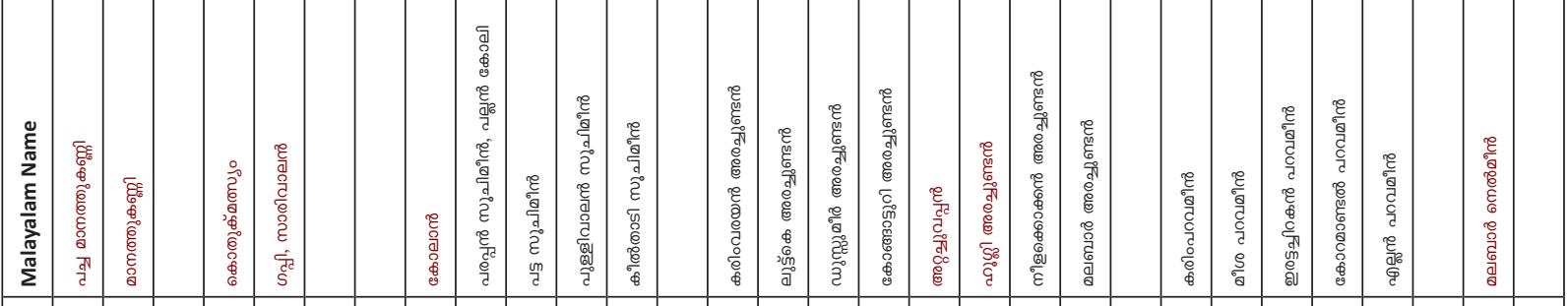

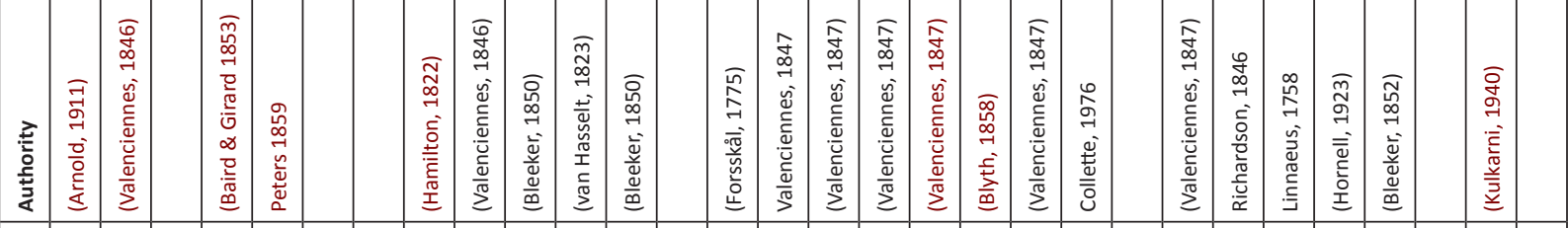

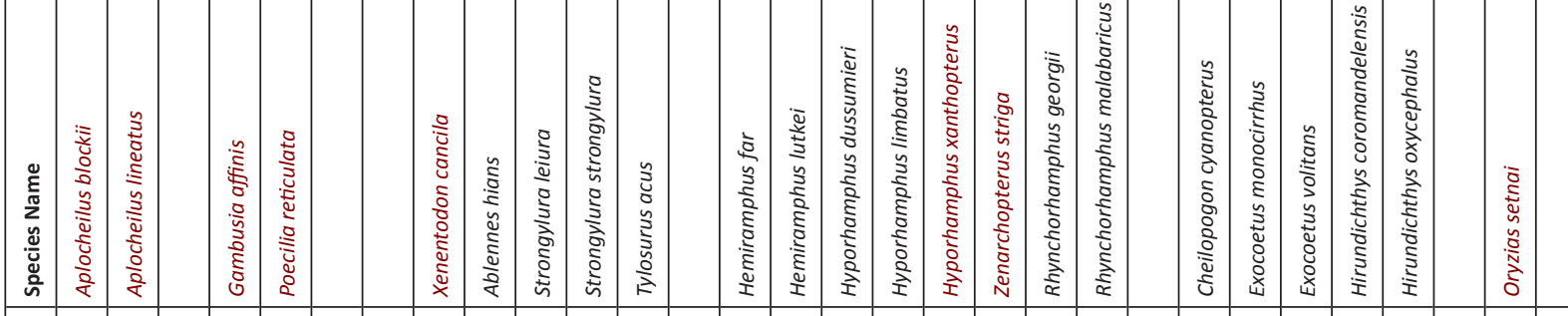

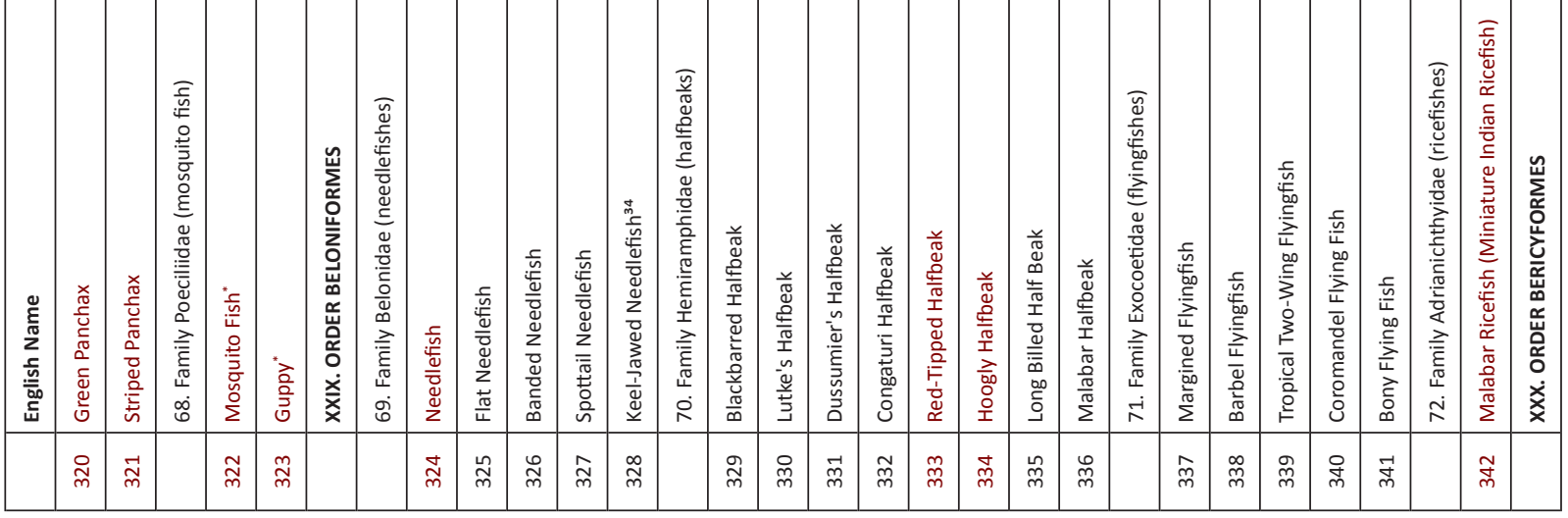




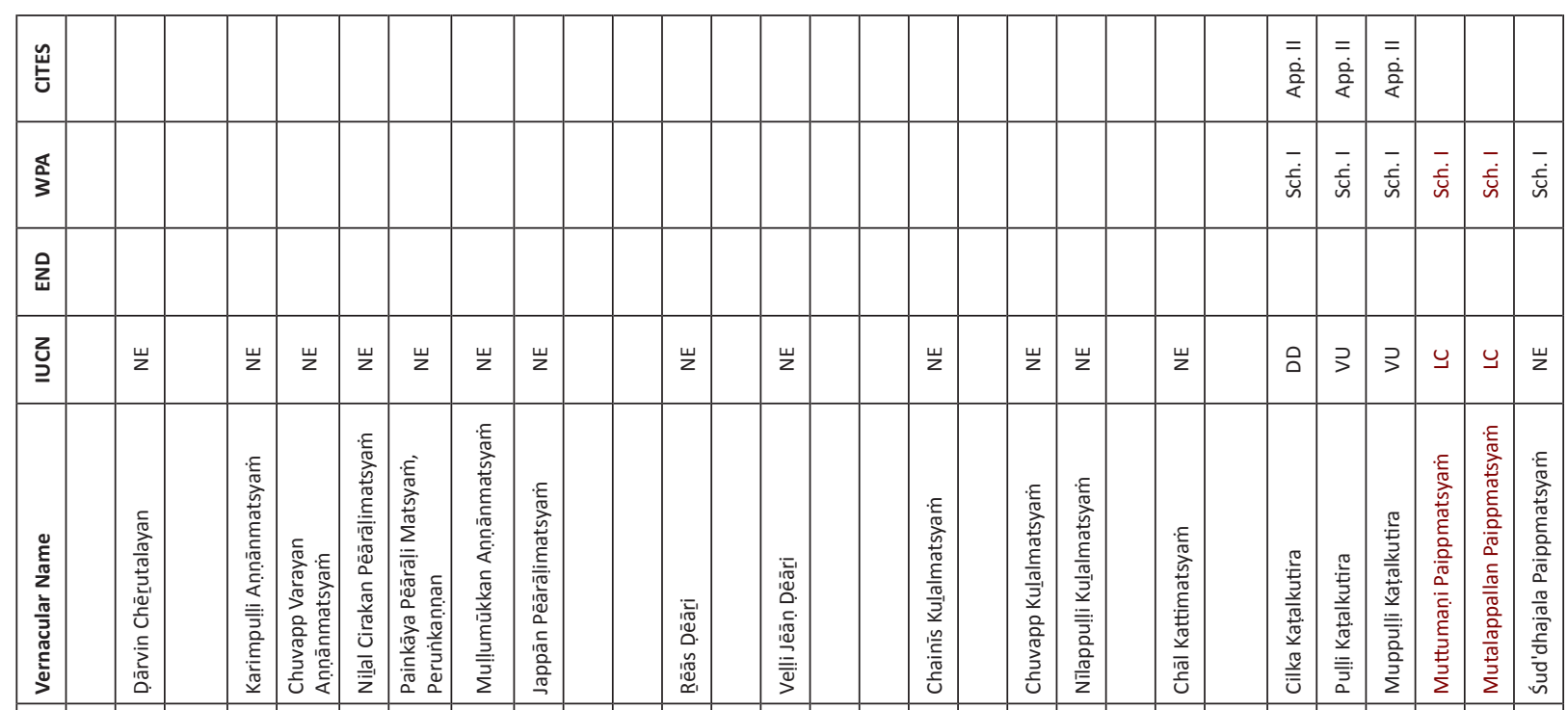

\begin{tabular}{|c|c|c|c|c|c|c|c|c|c|c|c|c|c|c|c|c|c|c|}
\hline 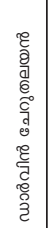 & 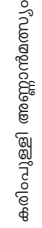 & 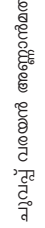 & 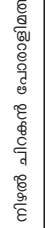 & 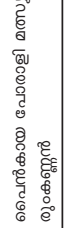 & 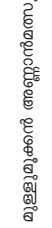 & 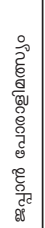 & 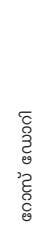 & 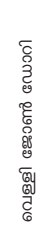 & 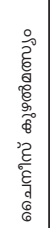 & 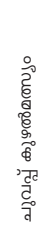 & 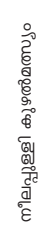 & 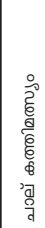 & 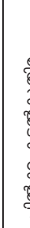 & & 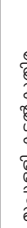 & 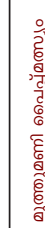 & & 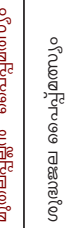 \\
\hline
\end{tabular}

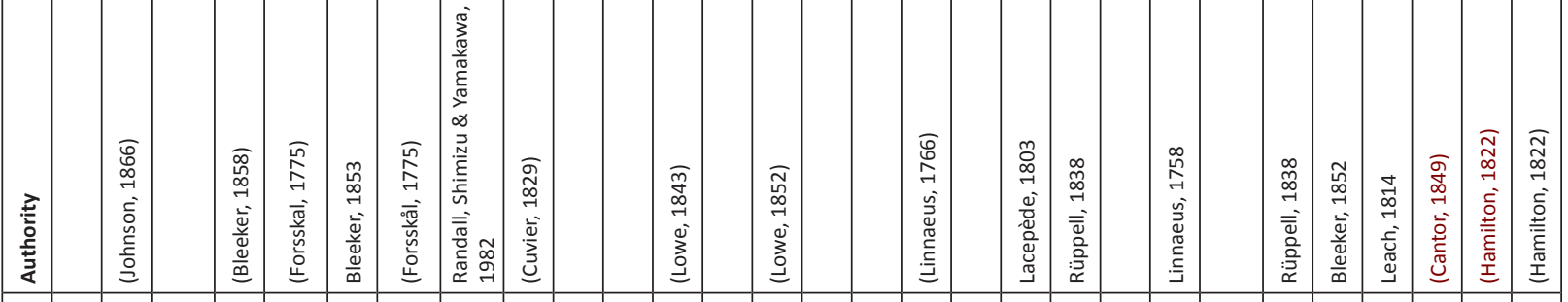

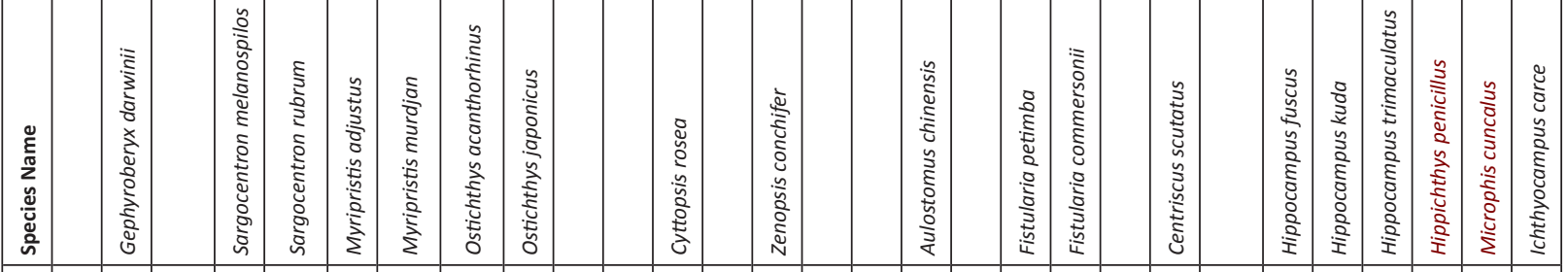

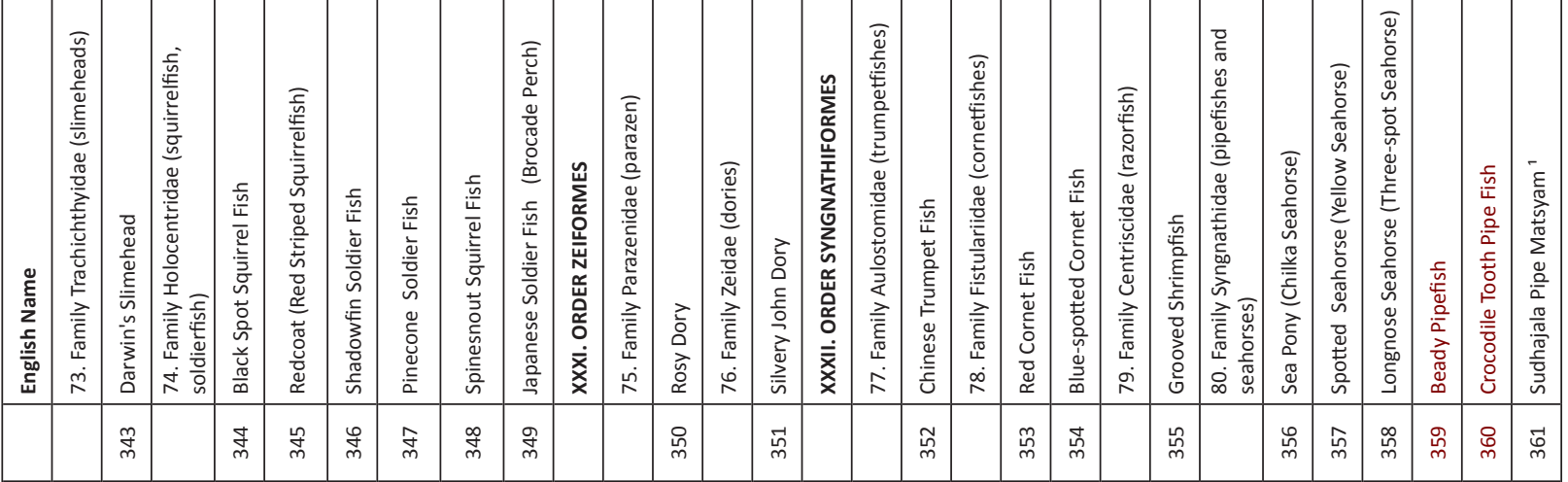




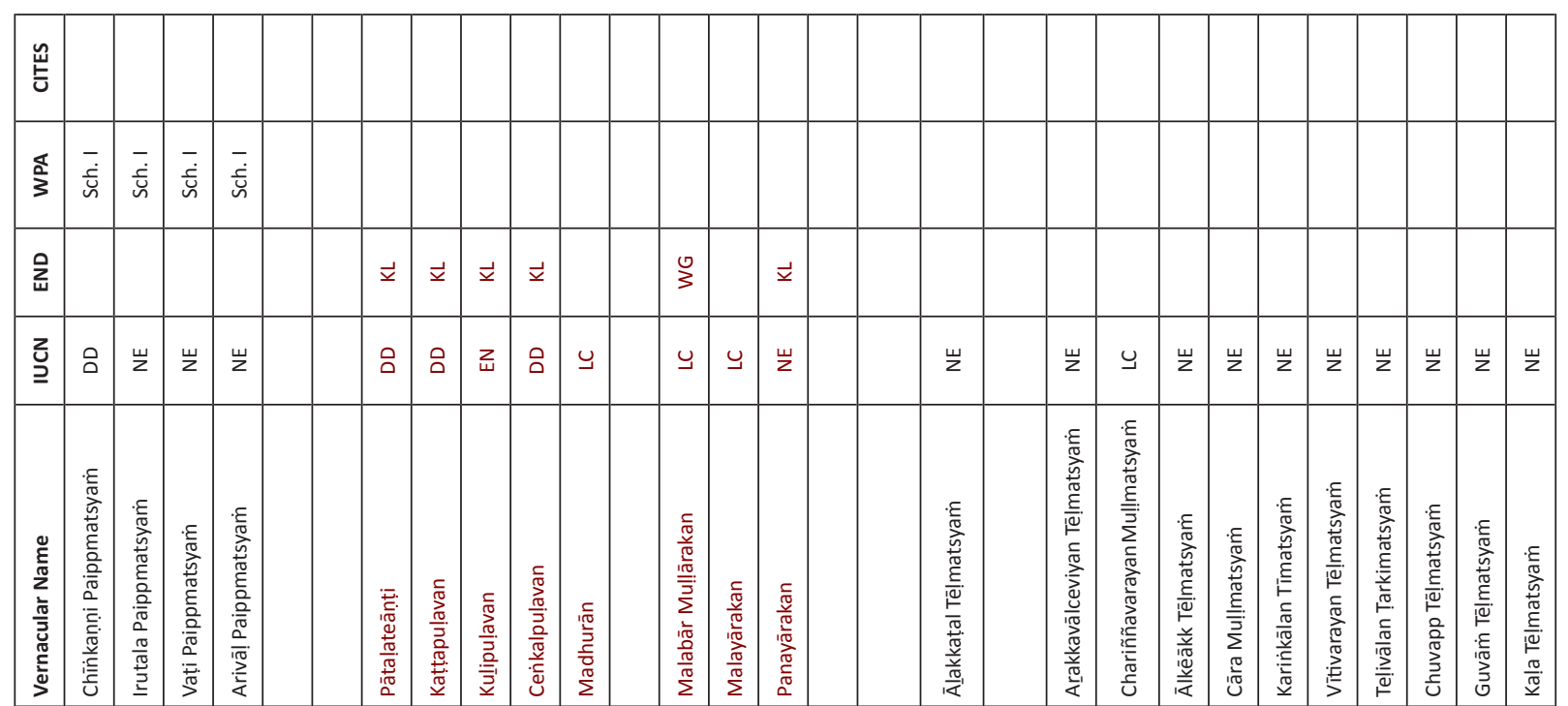

\begin{tabular}{|c|c|c|c|c|c|c|c|c|c|c|c|c|c|c|c|c|c|c|c|}
\hline & 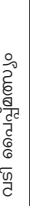 & 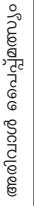 & & 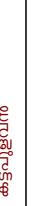 & 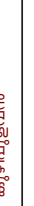 & 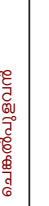 & 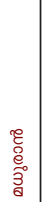 & 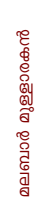 & 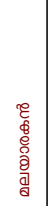 & 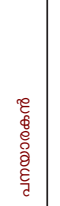 & 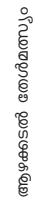 & 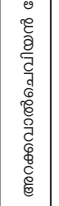 & 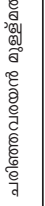 & 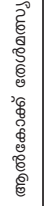 & 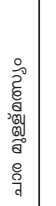 & & & 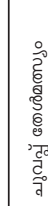 & 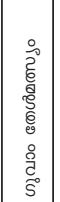 \\
\hline
\end{tabular}

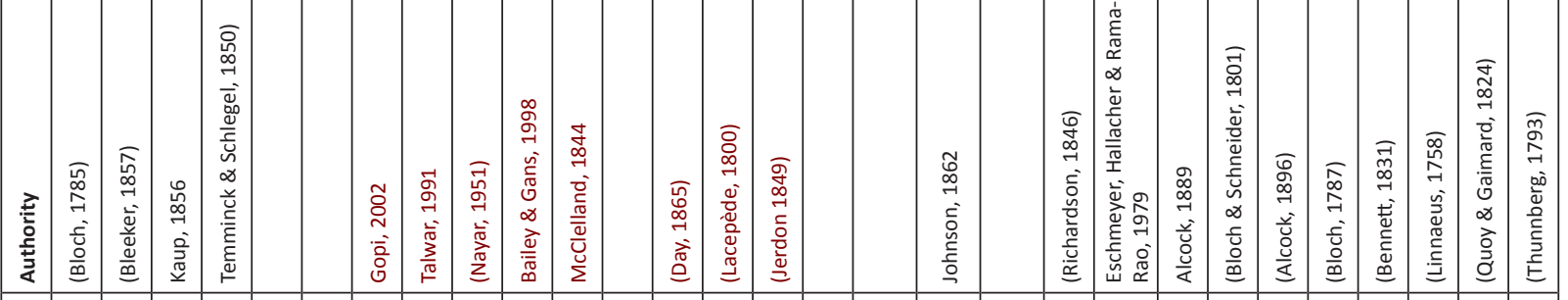

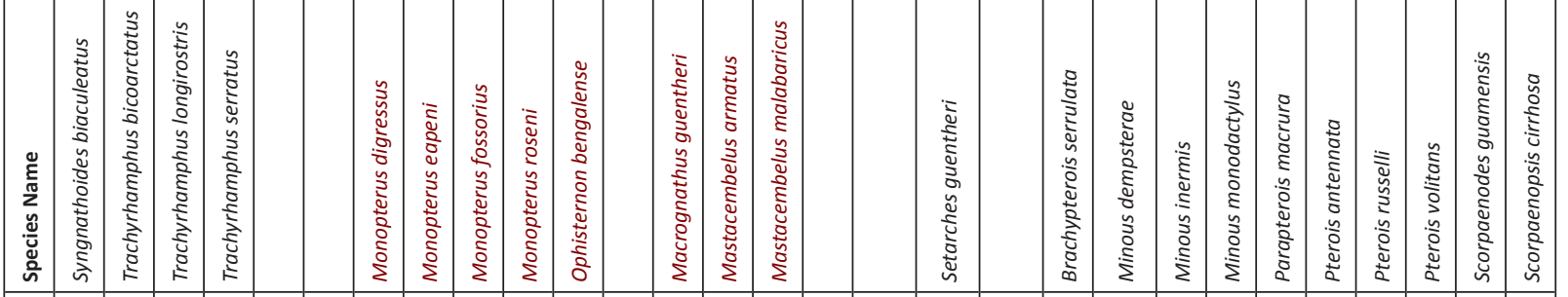

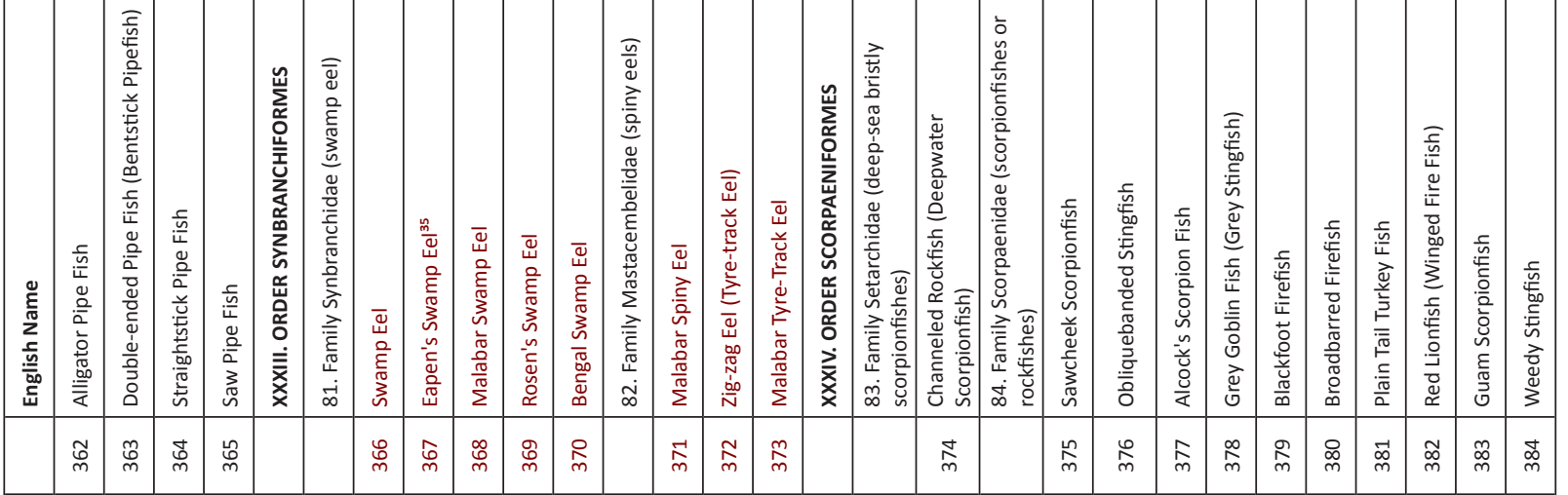




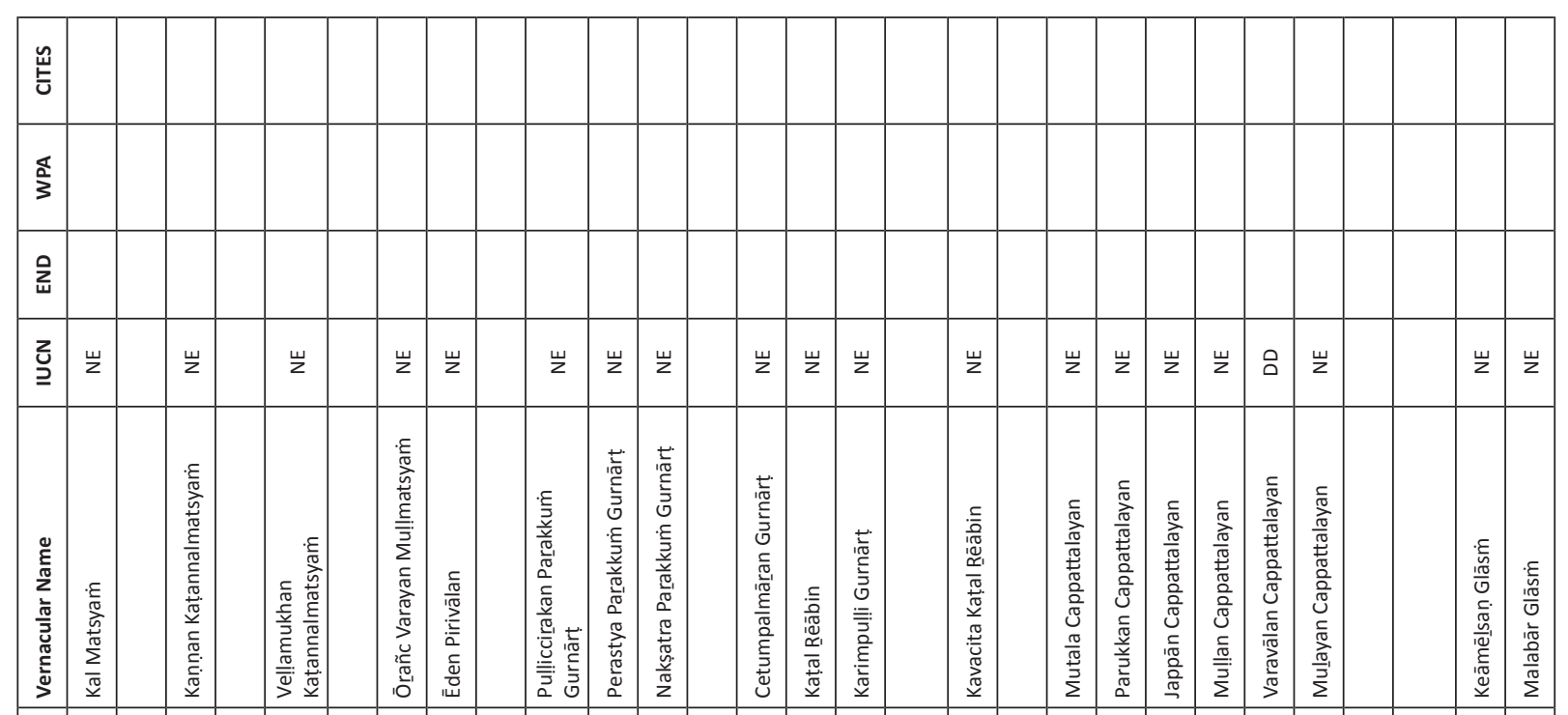

\begin{tabular}{|c|c|c|c|c|c|c|c|c|c|c|c|c|c|c|c|c|c|c|}
\hline 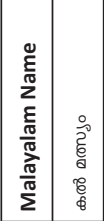 & 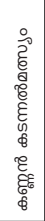 & है & 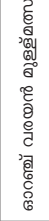 & 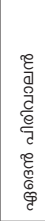 & 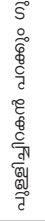 & 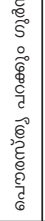 & 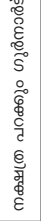 & 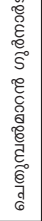 & 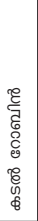 & 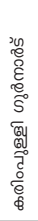 & 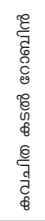 & 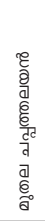 & 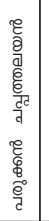 & 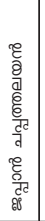 & & 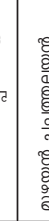 & & 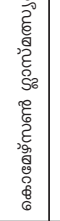 \\
\hline
\end{tabular}

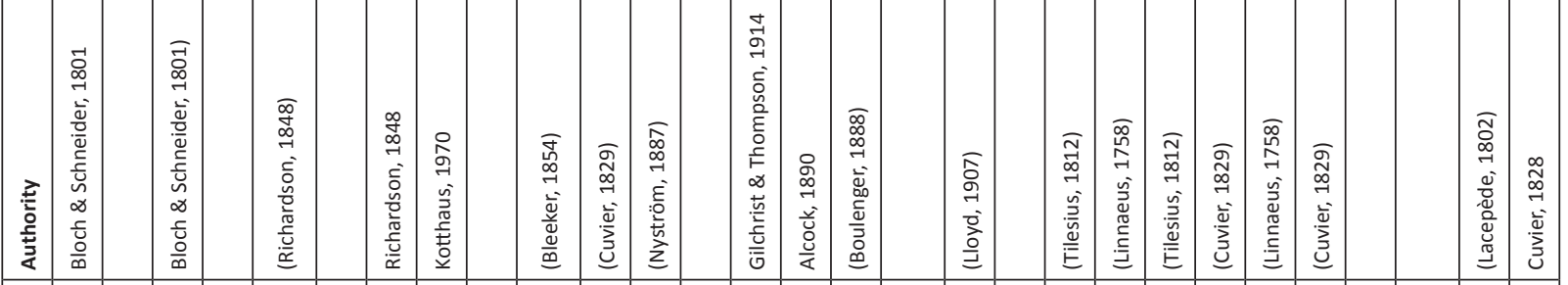

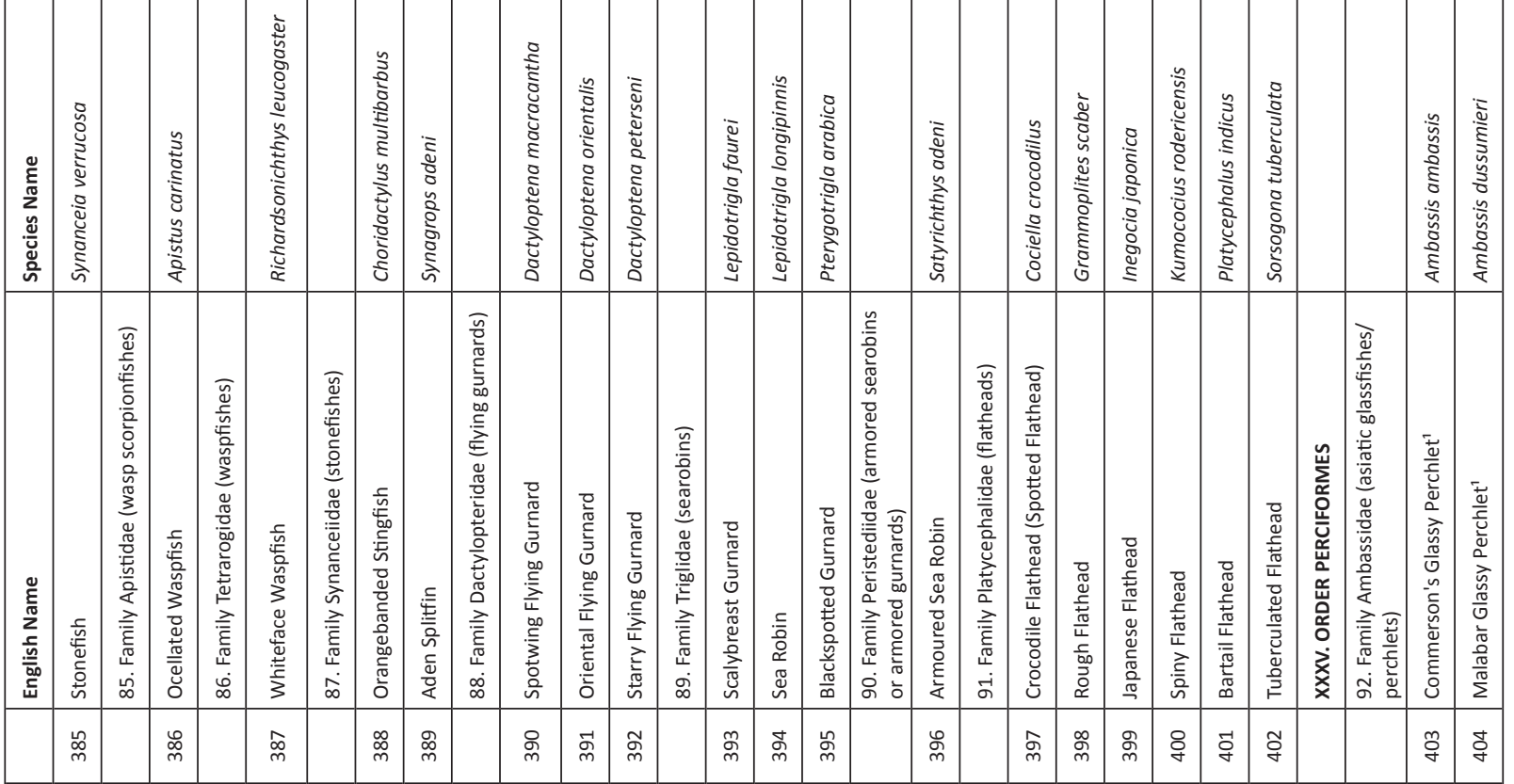




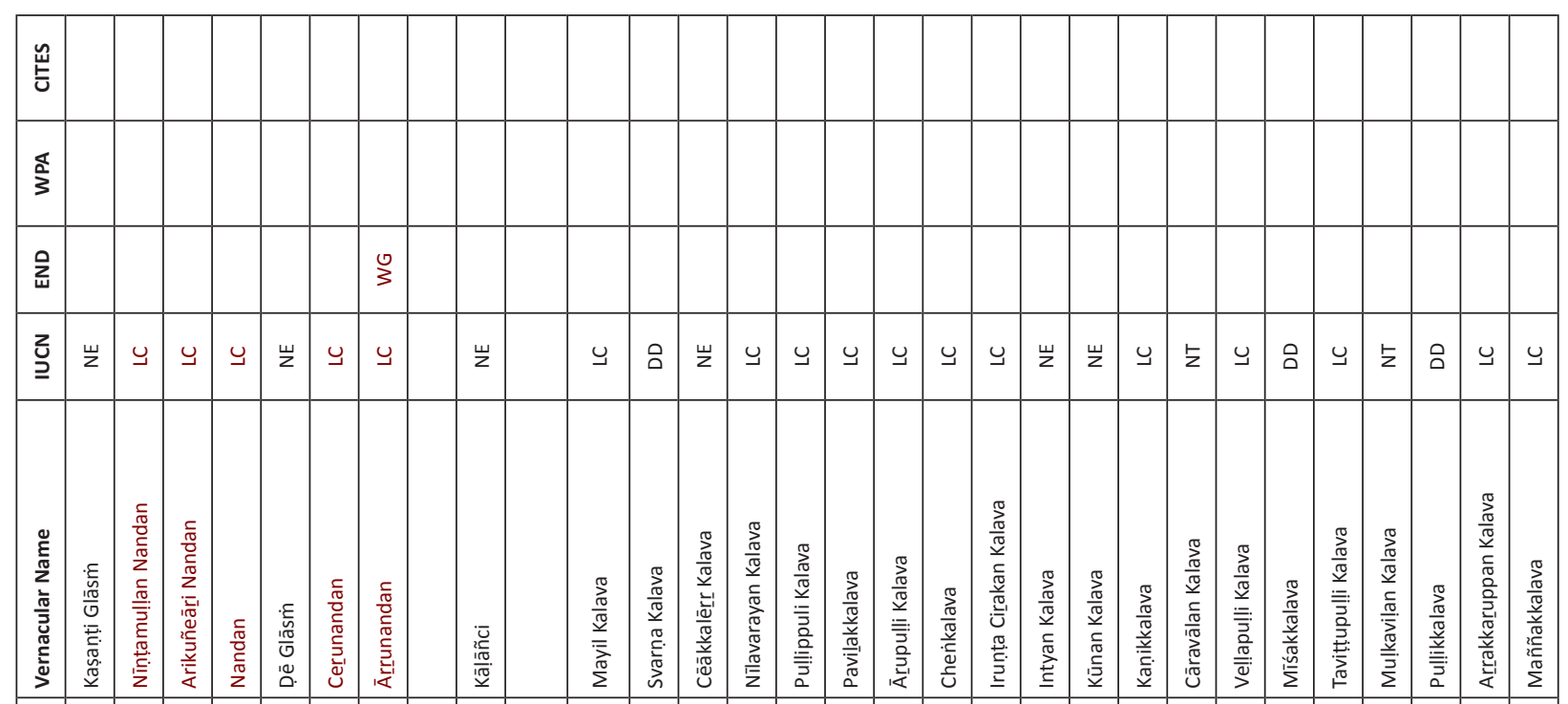

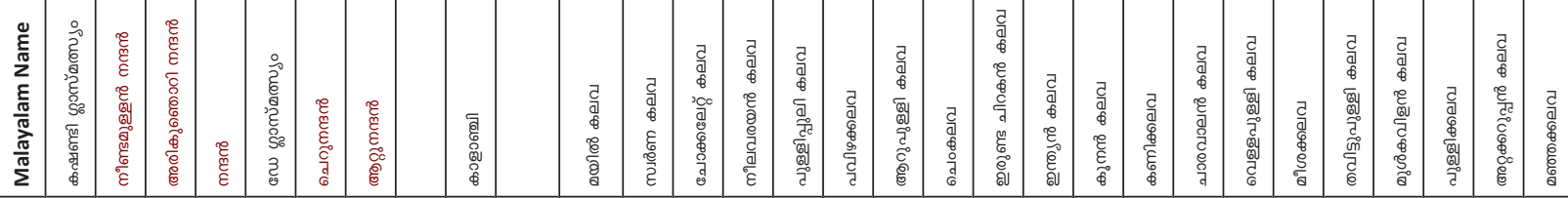

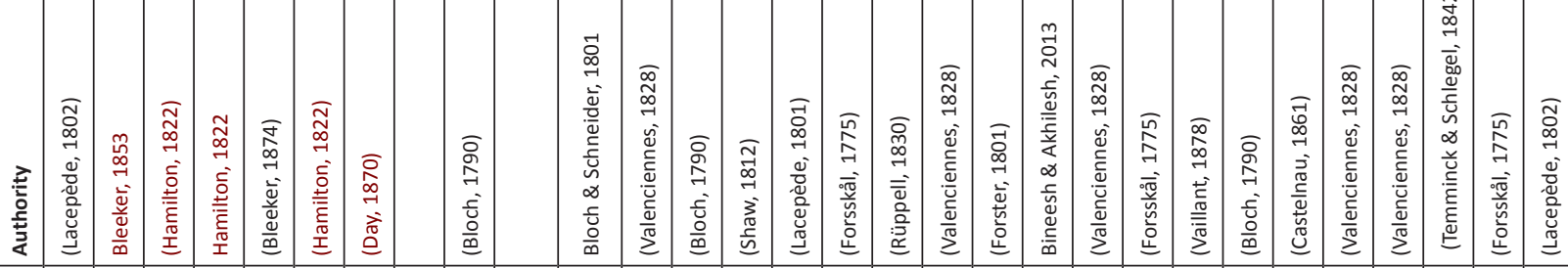

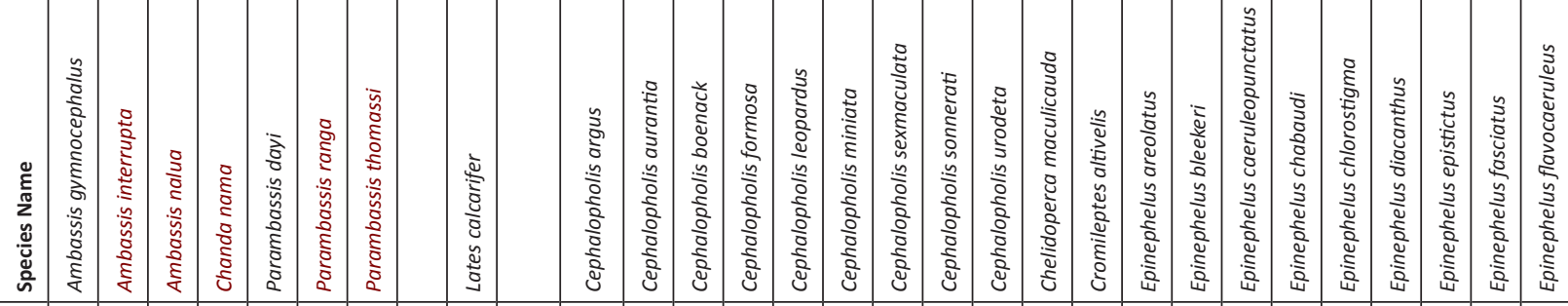

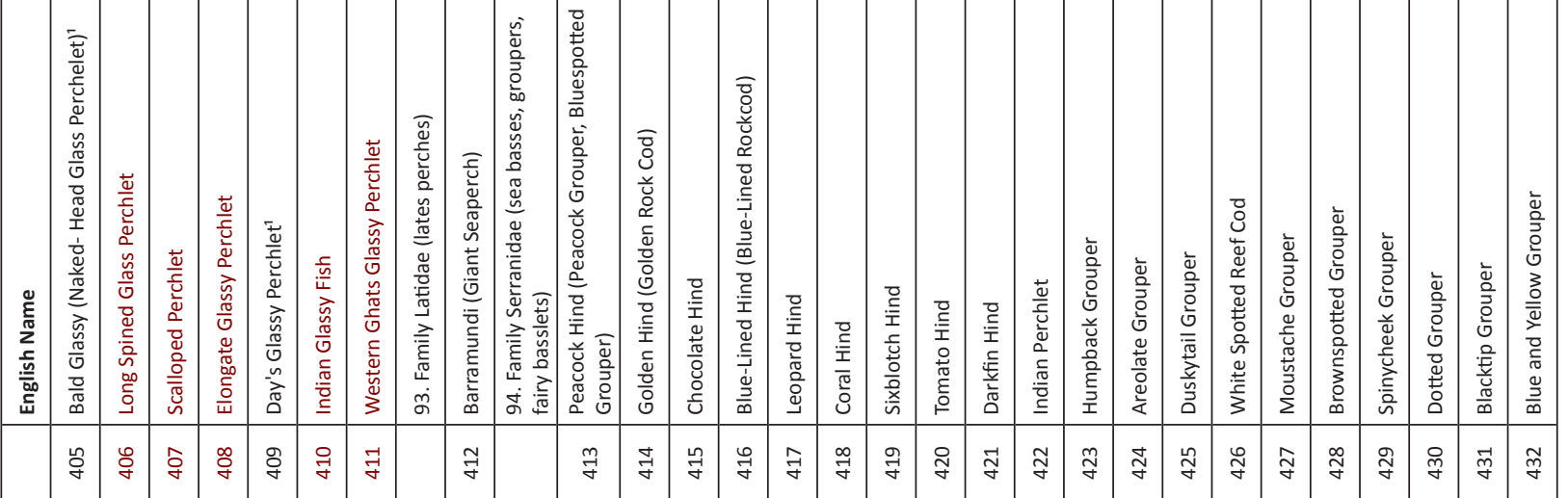




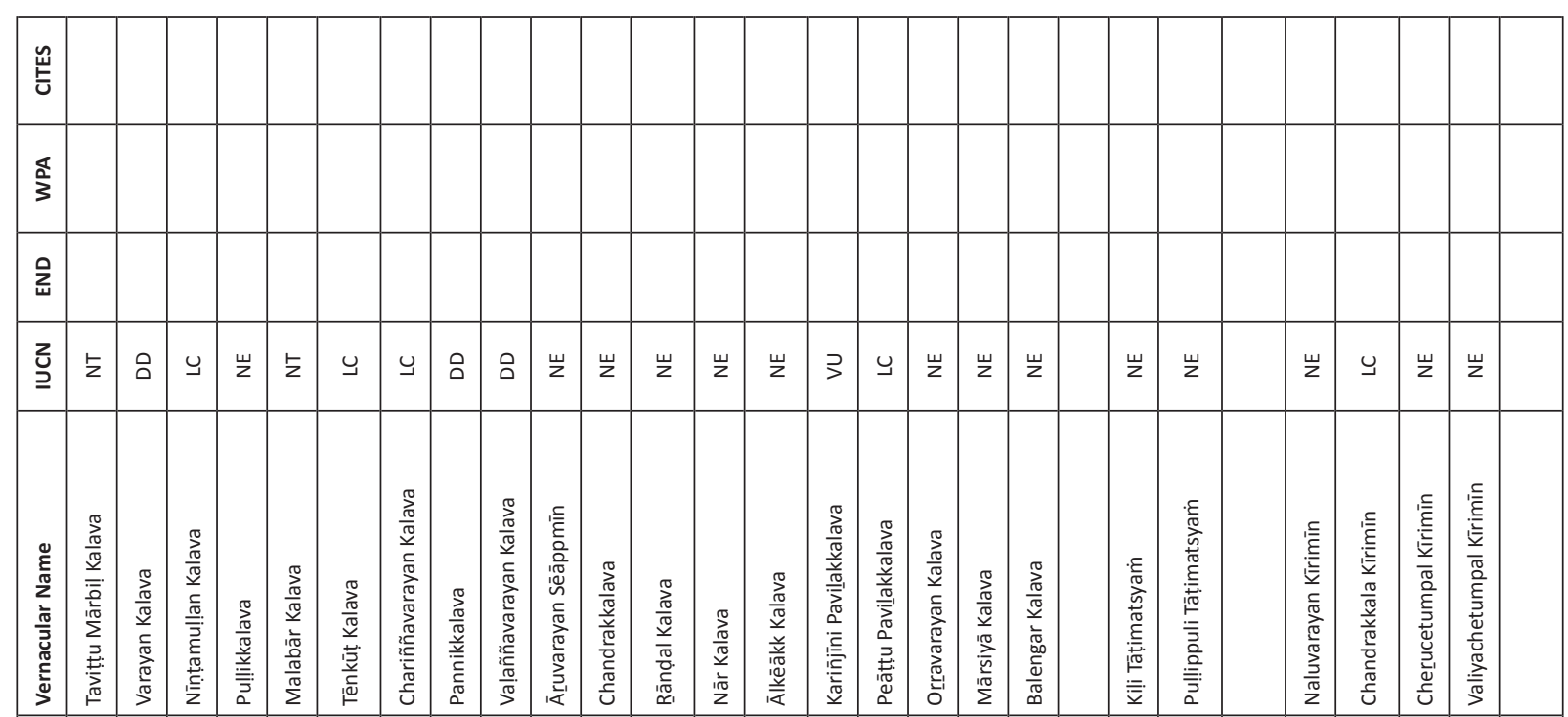

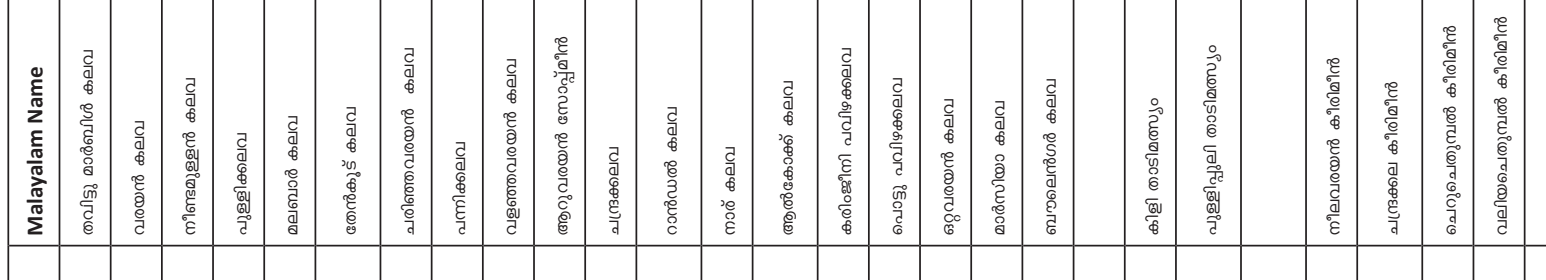

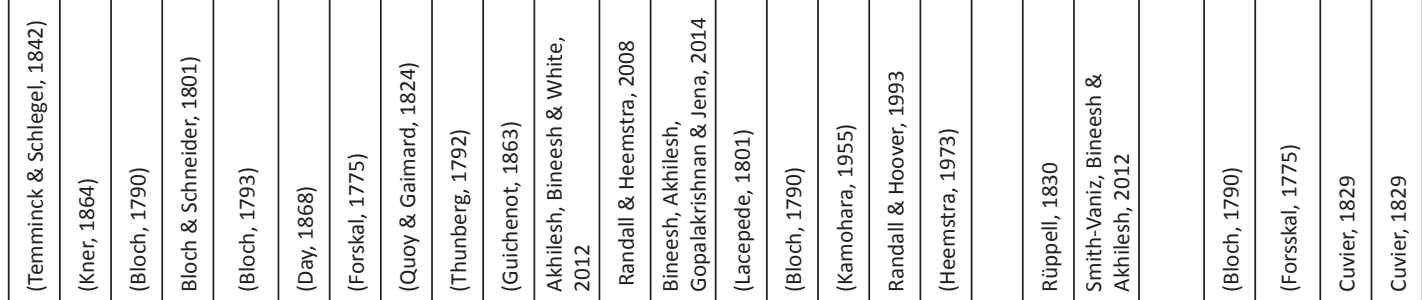
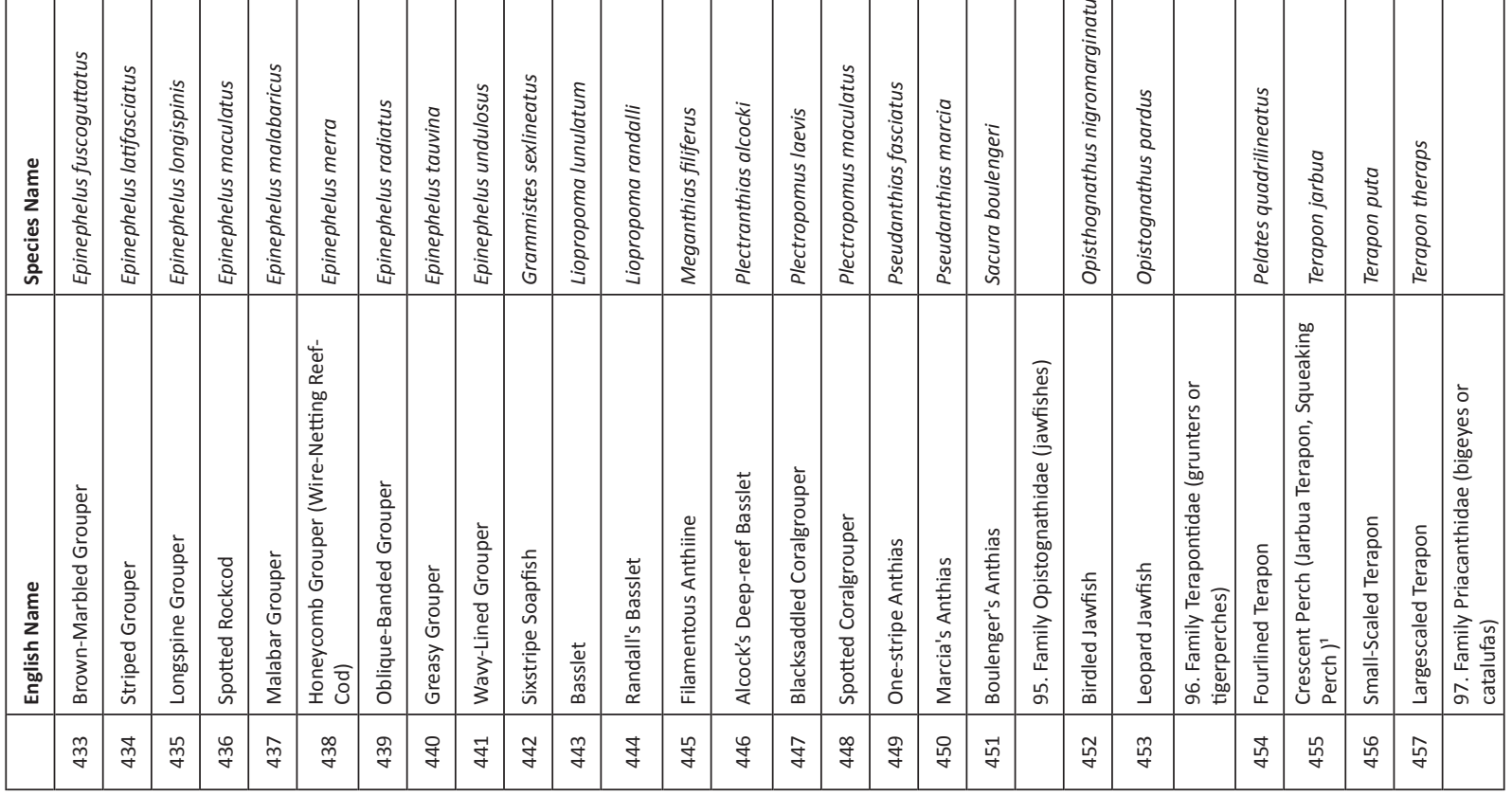


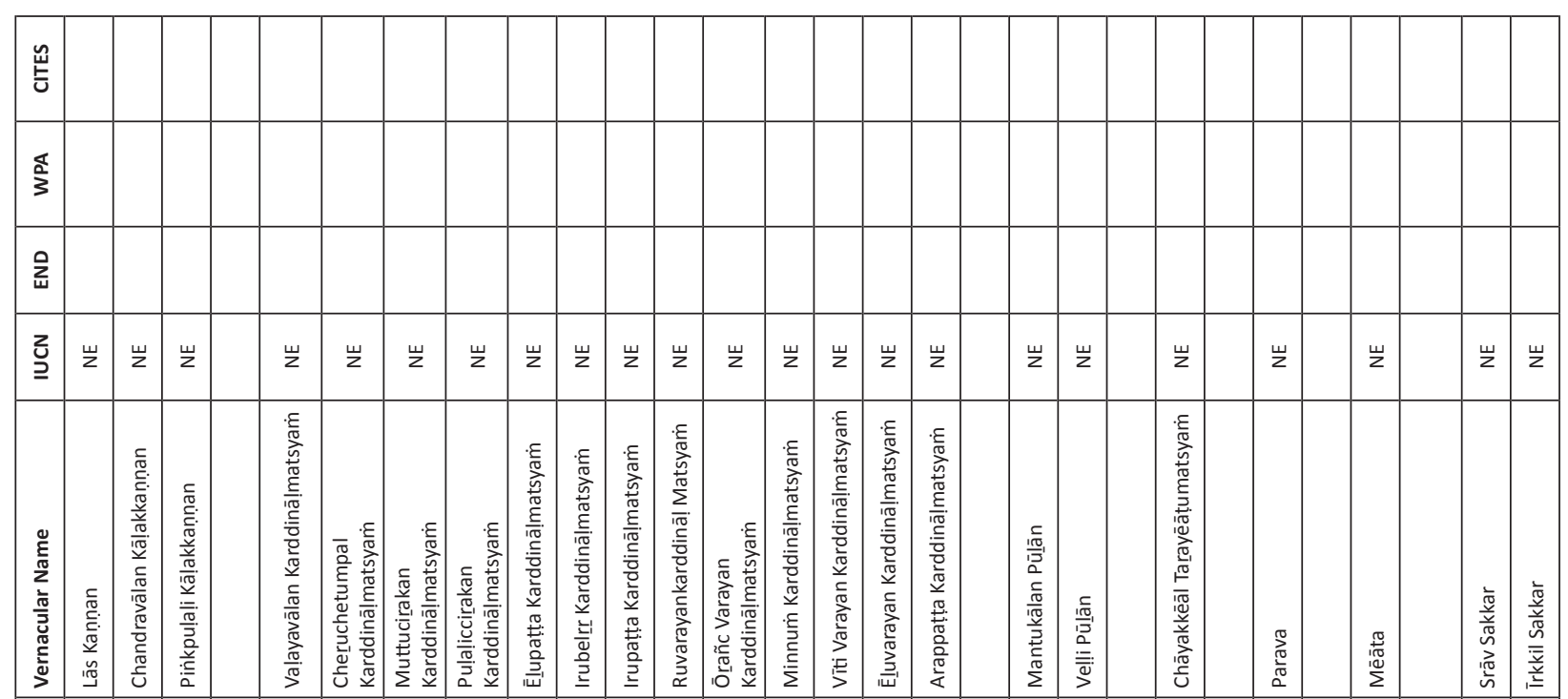

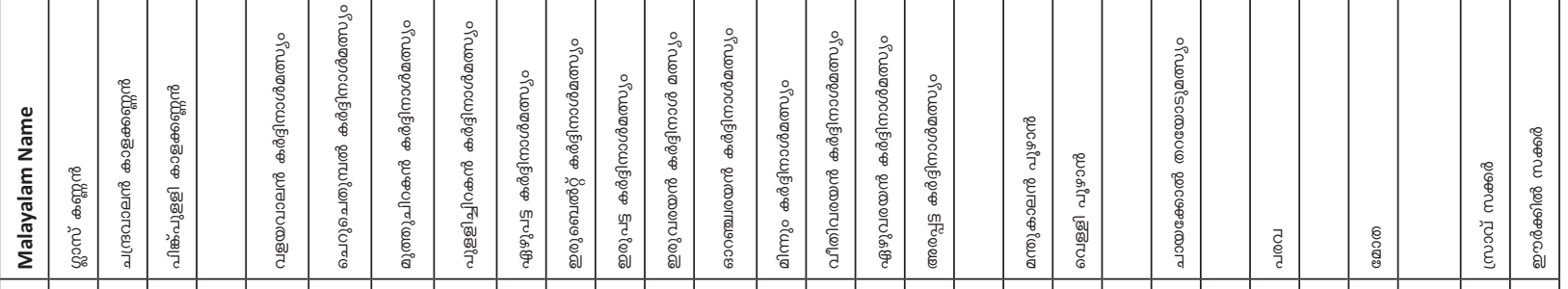

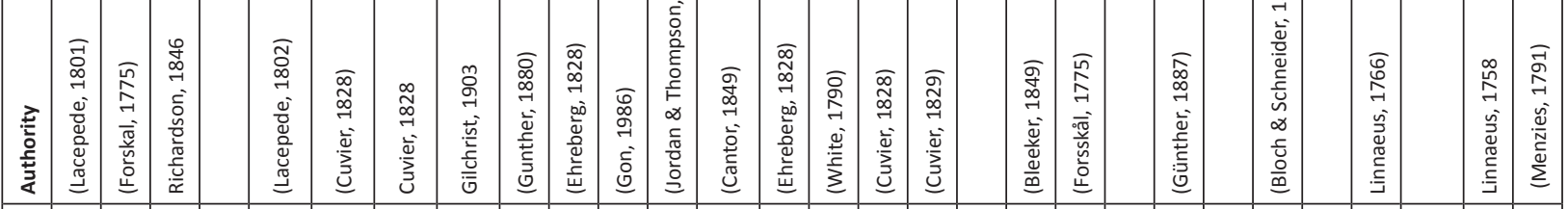

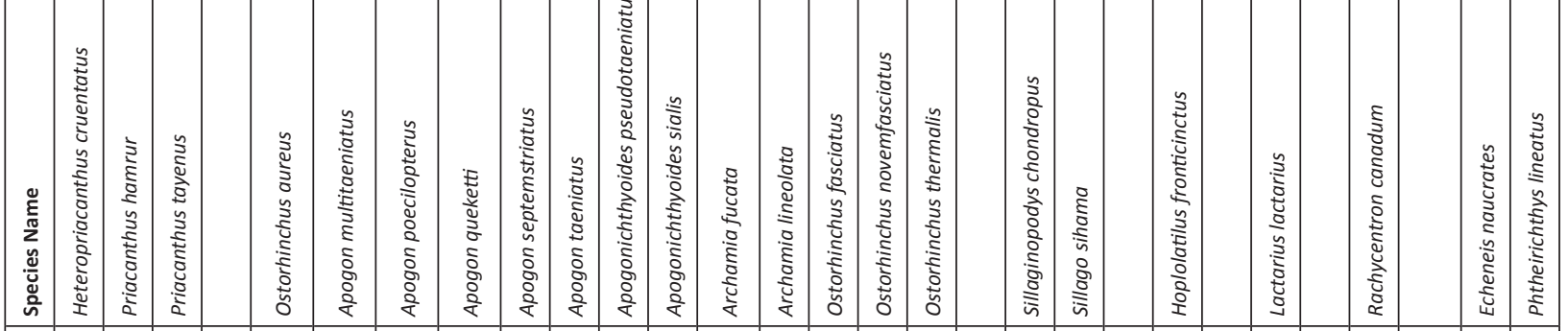

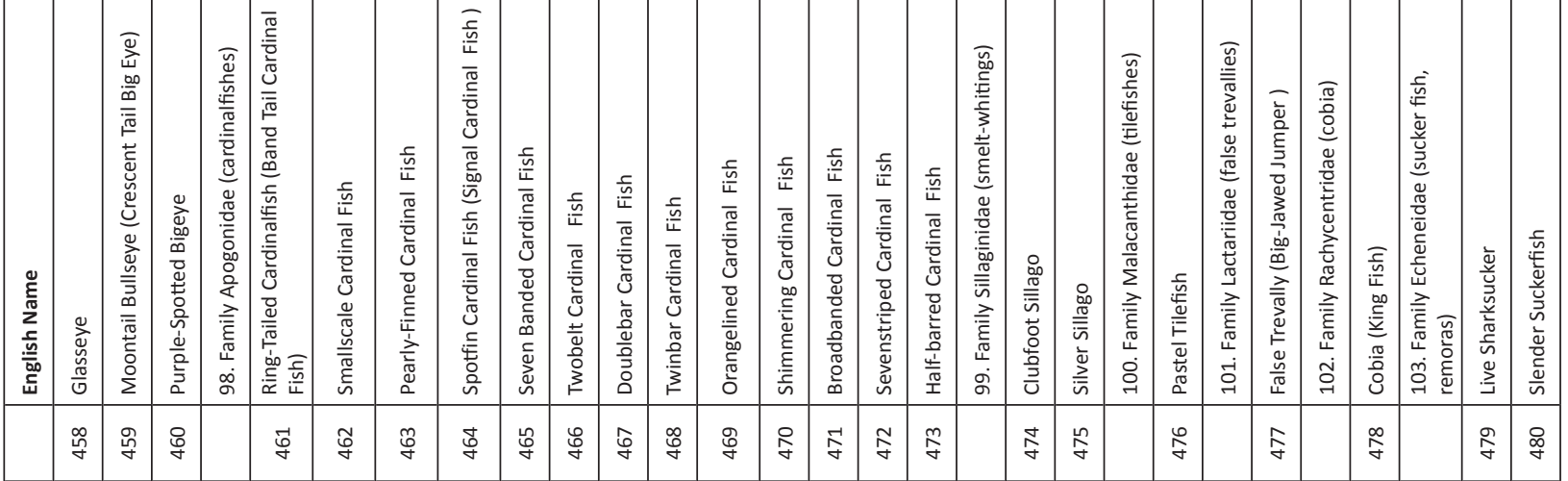




\begin{tabular}{|c|c|c|c|c|c|c|c|c|c|c|c|c|c|c|c|c|c|c|c|c|c|c|c|c|c|c|c|c|c|}
\hline 峞 & & & & & & & & & & & & & & & & & & & & & & & & & & & & & \\
\hline$\hat{s}^{\alpha}$ & & & & & & & & & & & & & & & & & & & & & & & & & & & & & \\
\hline 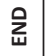 & & & & & & & & & & & & & & & & & & & & & & & & & & & & & \\
\hline בֶ & y & $u$ & 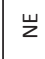 & 崩 & 㟧 & 㟧 & $\underline{y}$ & 㟧 & 岂 & 㟧 & 崩 & 崩 & 岁 & 㟧 & 㟧 & 崩 & 㟧 & 崩 & 㟧 & 岁 & $\underline{\underline{z}}$ & w & $\underline{\underline{z}}$ & $\vec{w}$ & $\underline{u}$ & 岁 & w & צ & 崩 \\
\hline 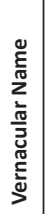 & 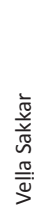 & 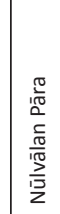 & 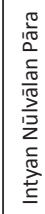 & 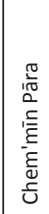 & 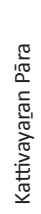 & 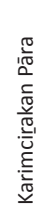 & 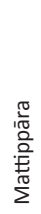 & 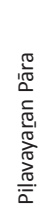 & 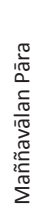 & 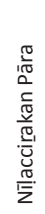 & 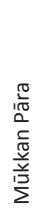 & 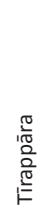 & $\begin{array}{l}\frac{0}{10} \\
\frac{0}{0}\end{array}$ & 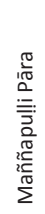 & 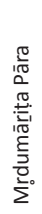 & 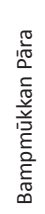 & 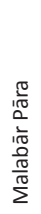 & 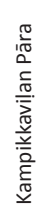 & 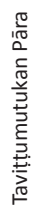 & 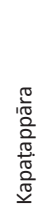 & 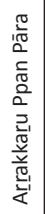 & 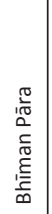 & 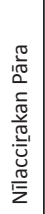 & 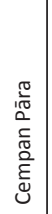 & 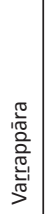 & 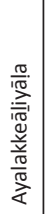 & 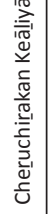 & 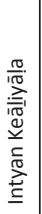 & 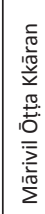 \\
\hline
\end{tabular}

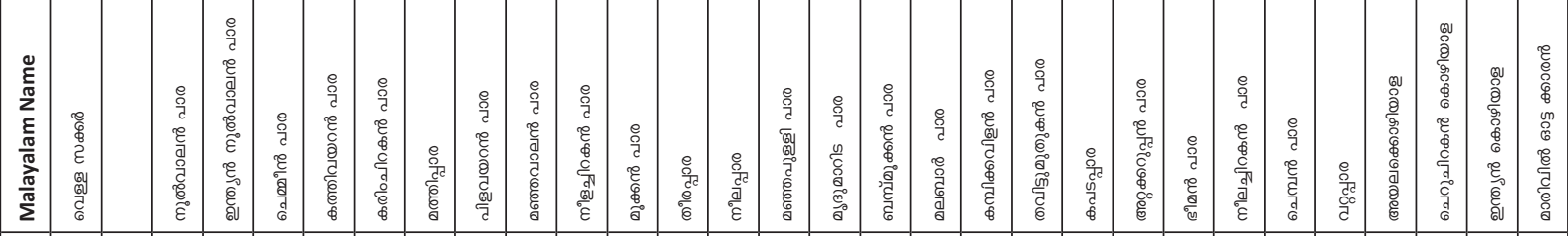

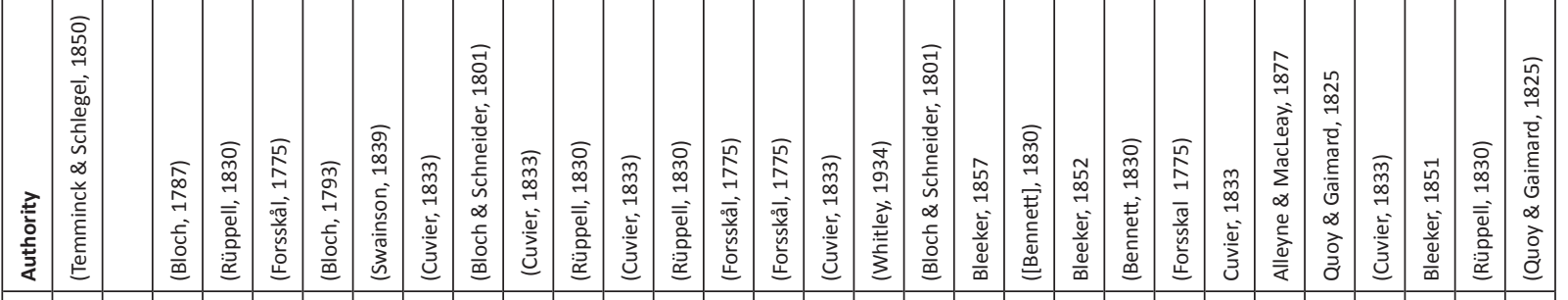

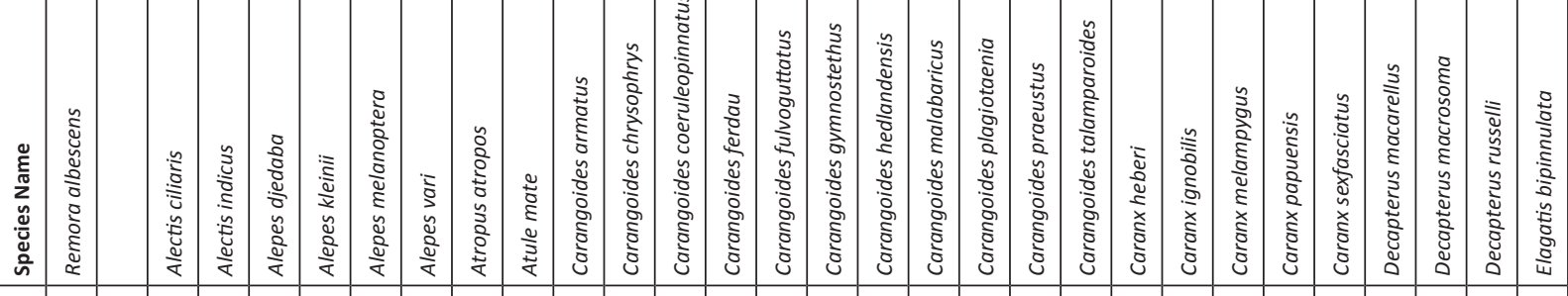

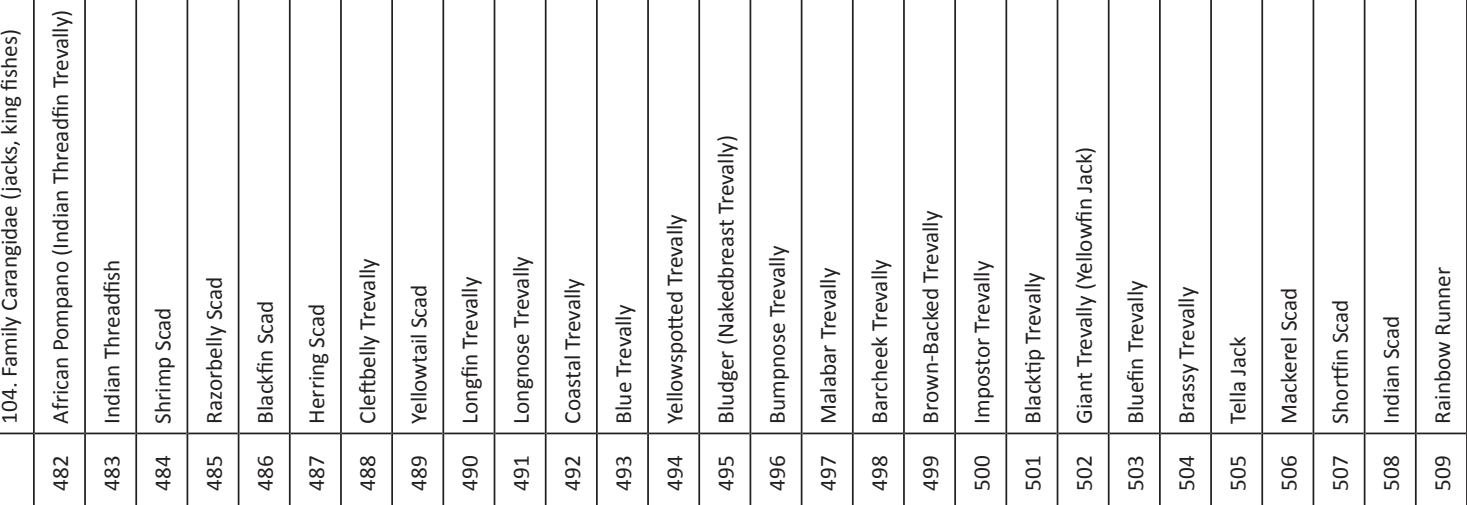




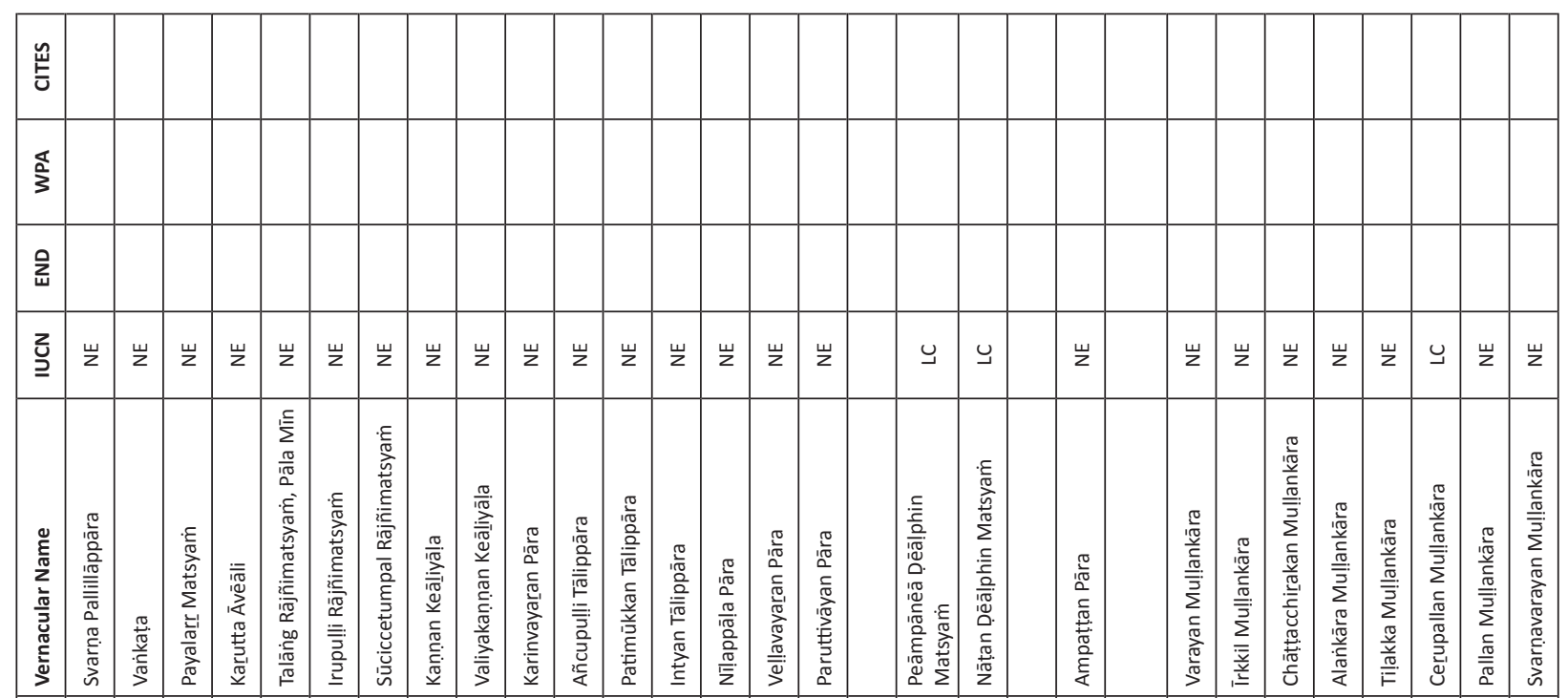

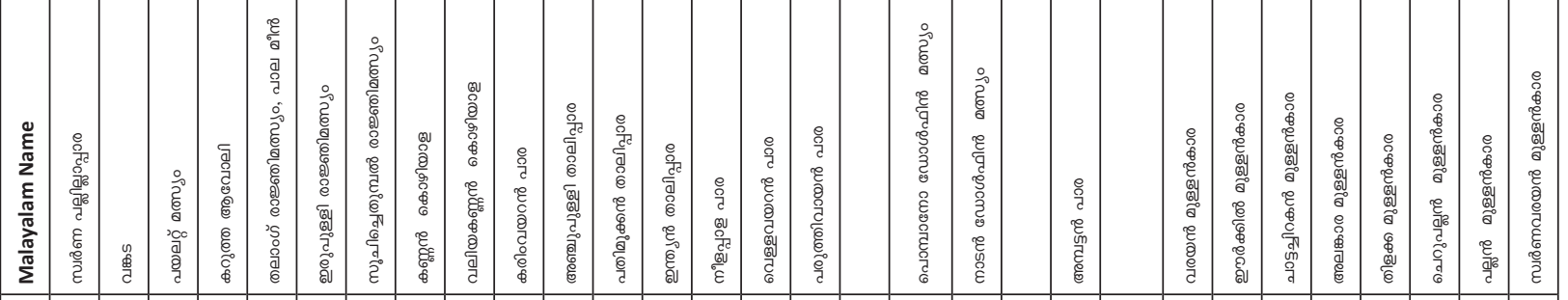

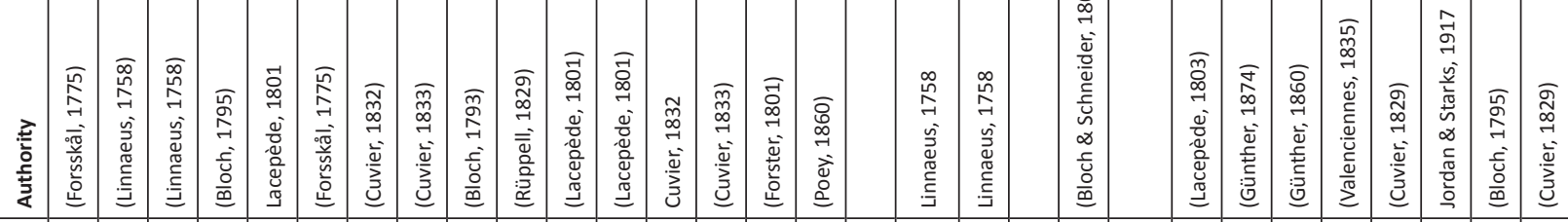

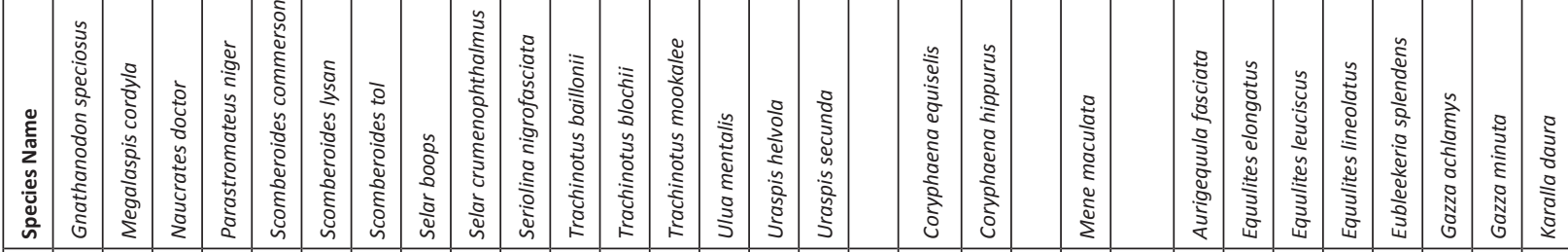

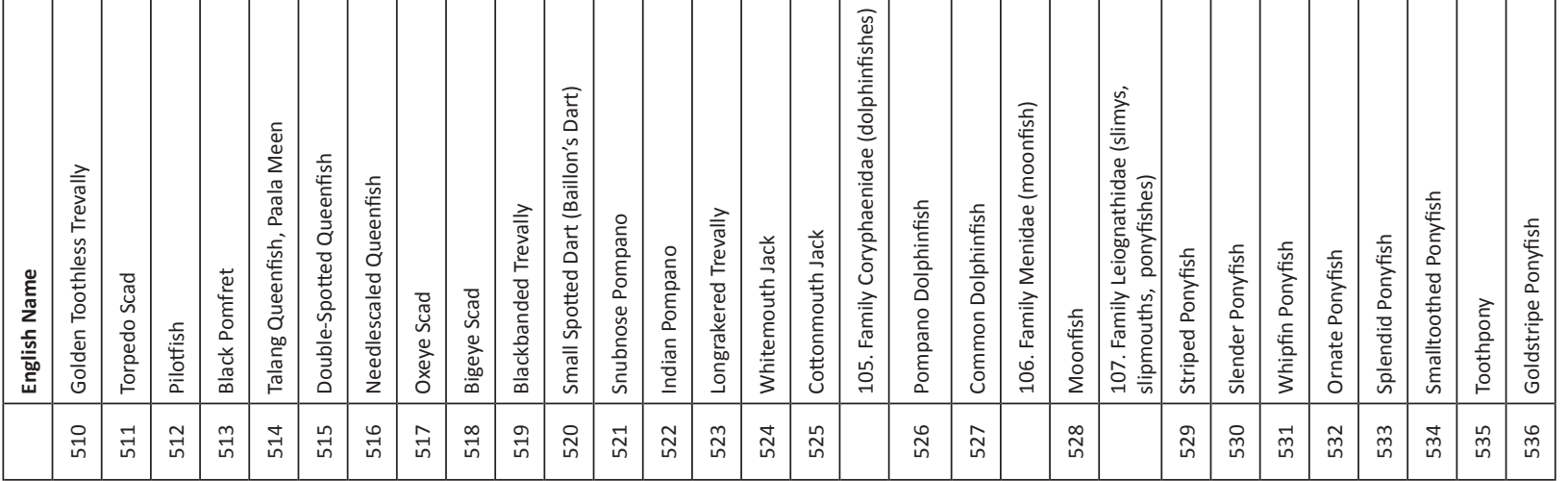




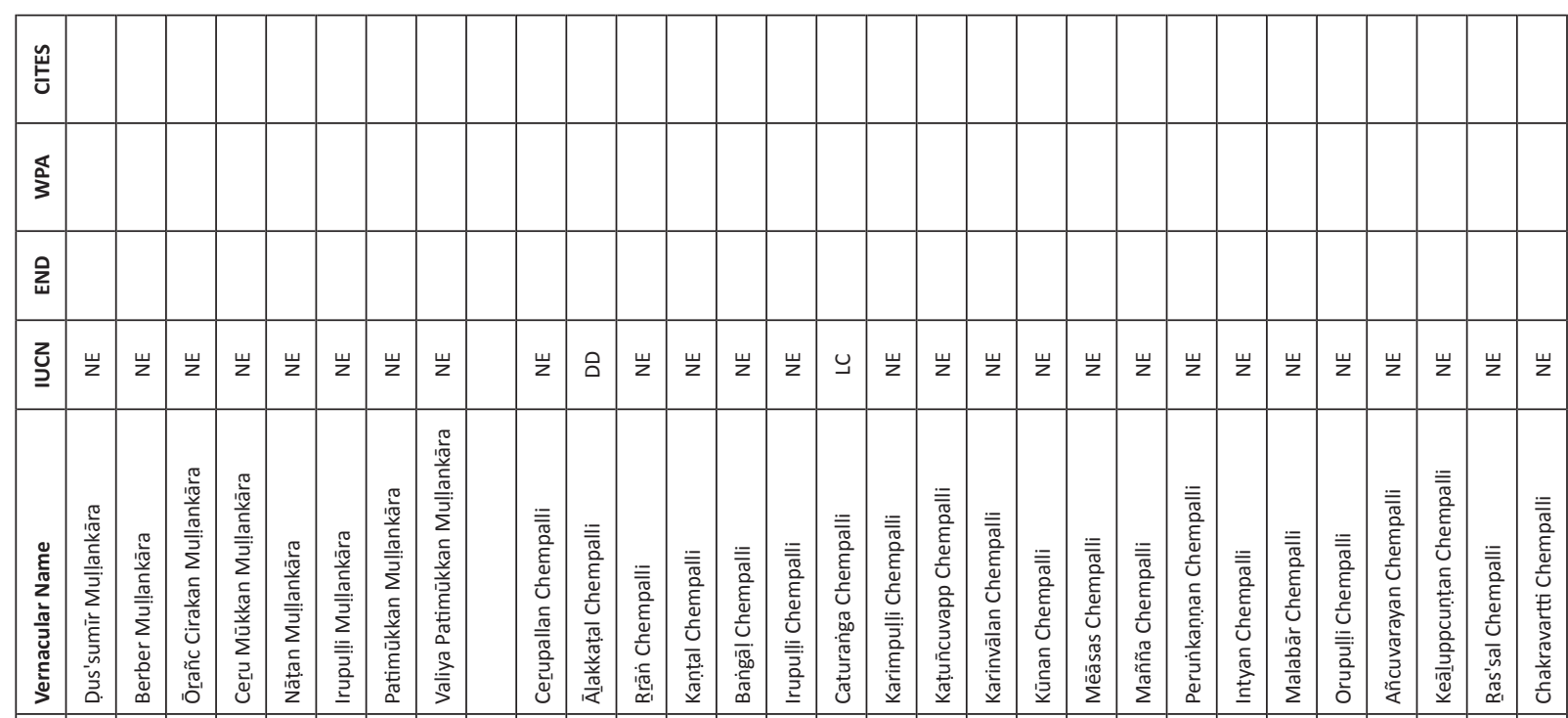

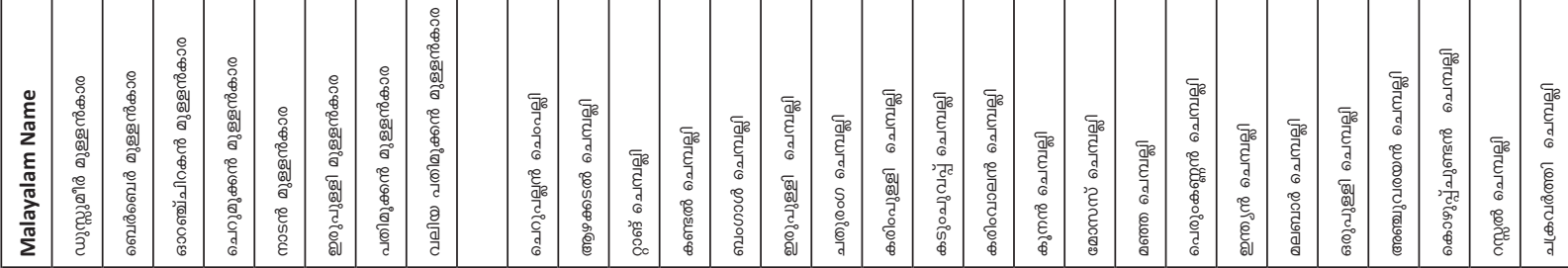

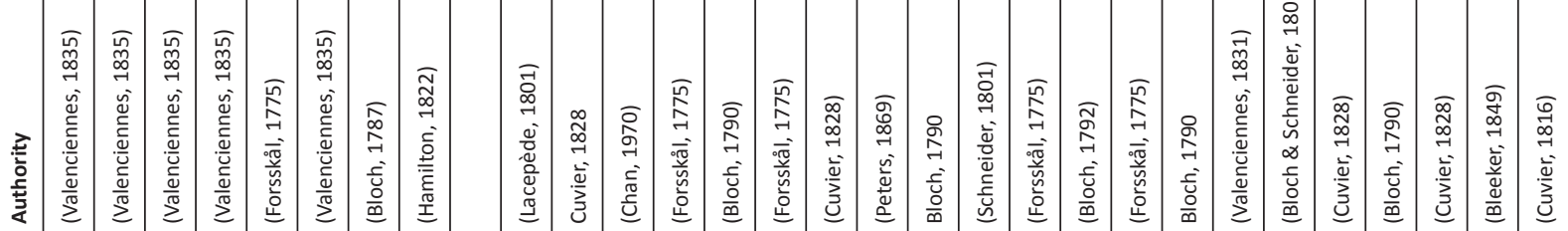

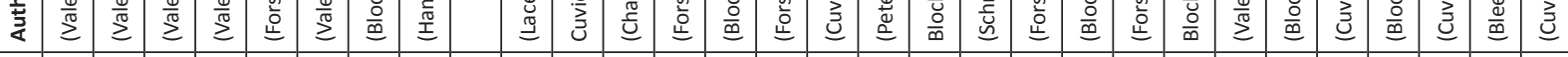

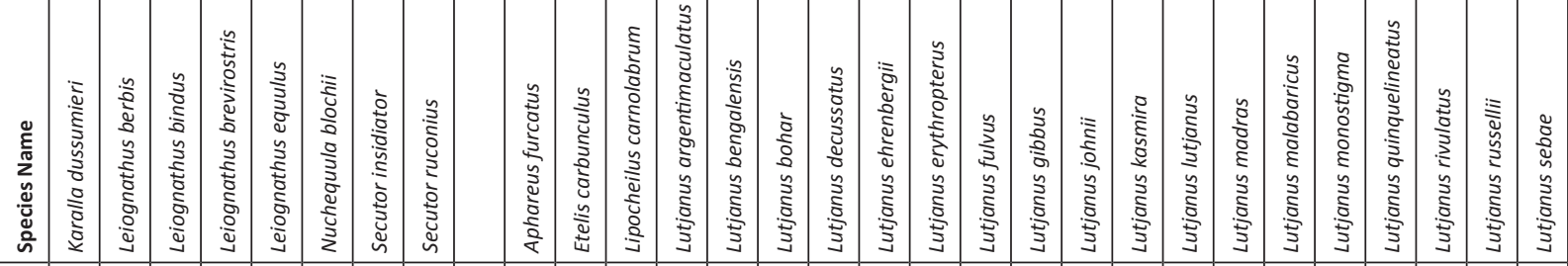

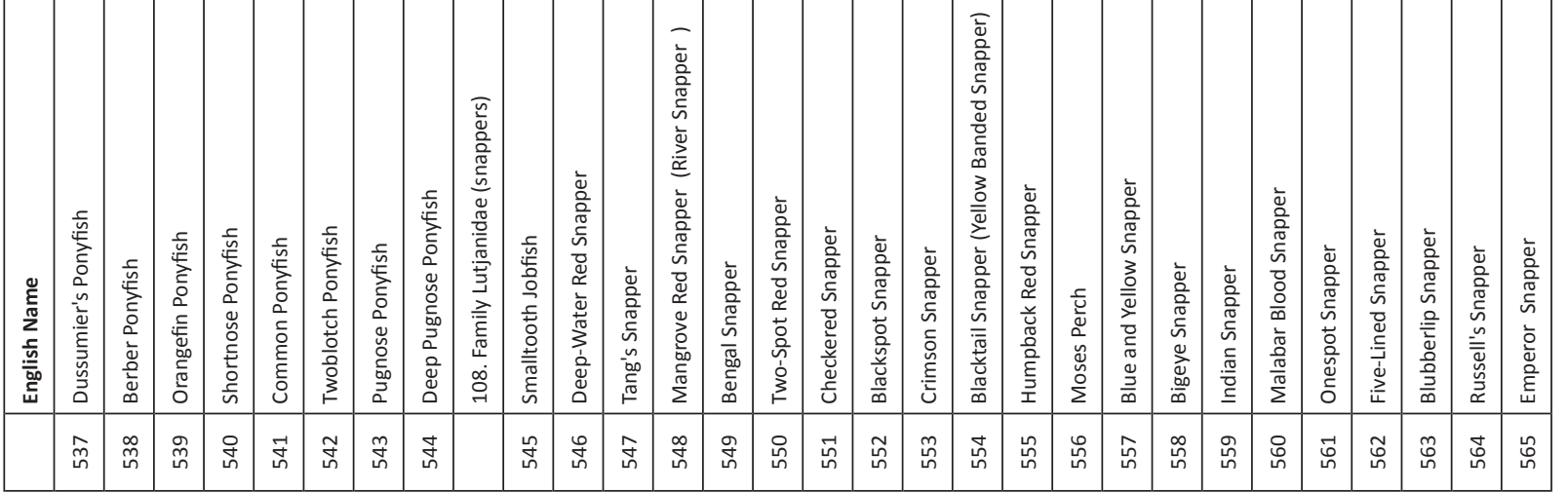




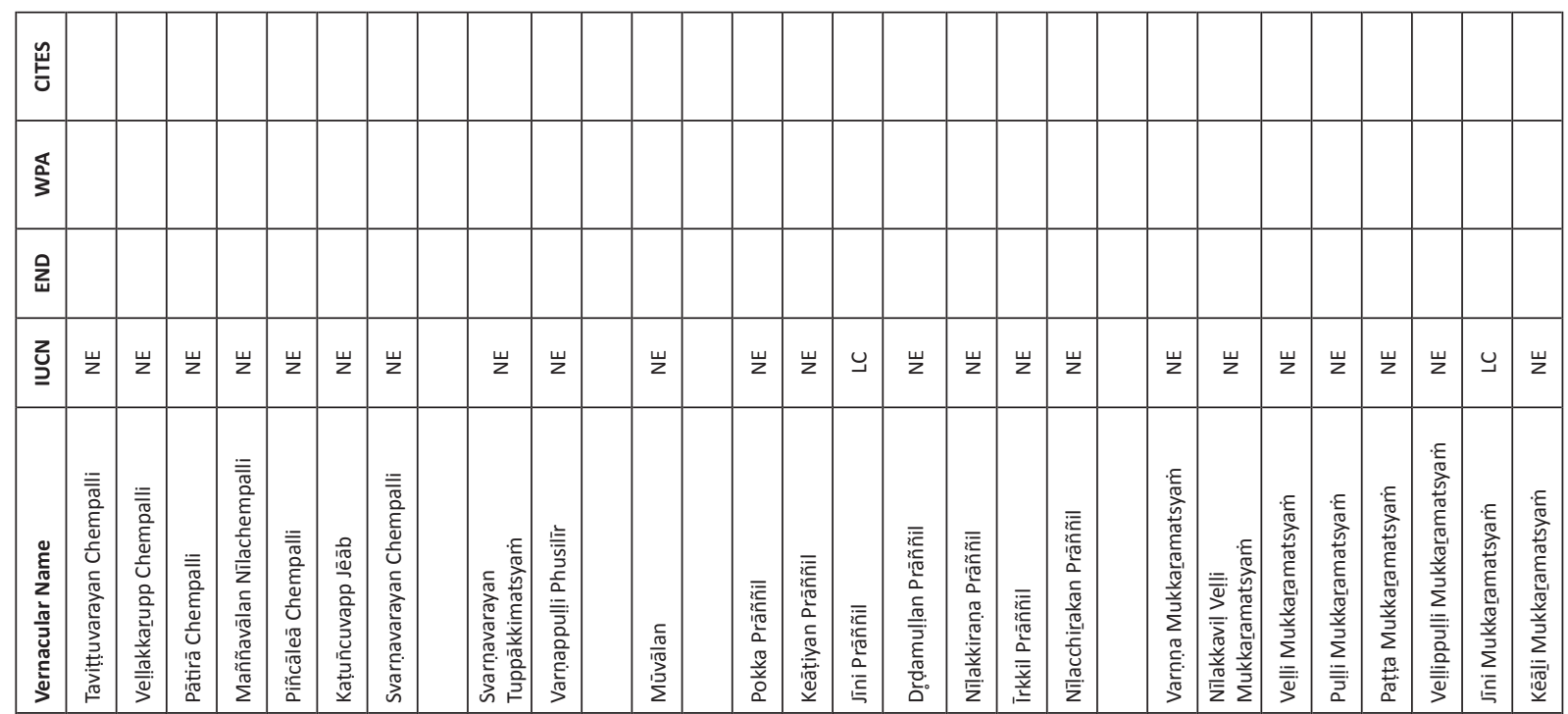

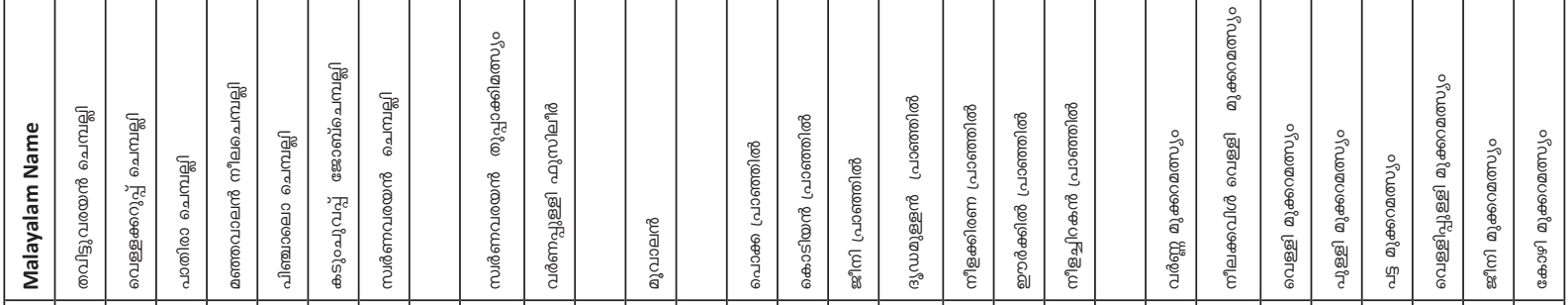

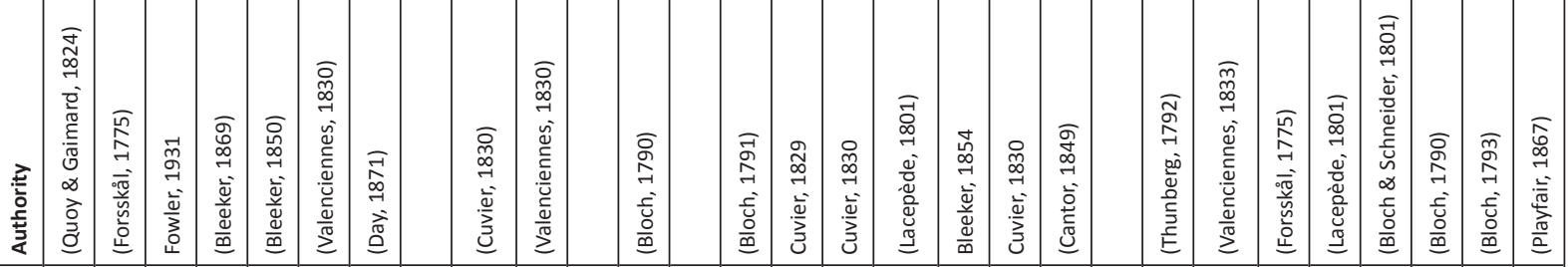

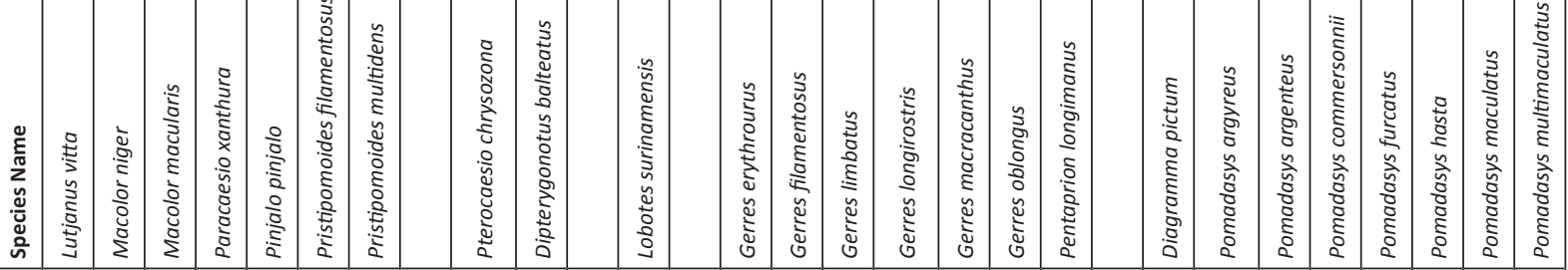

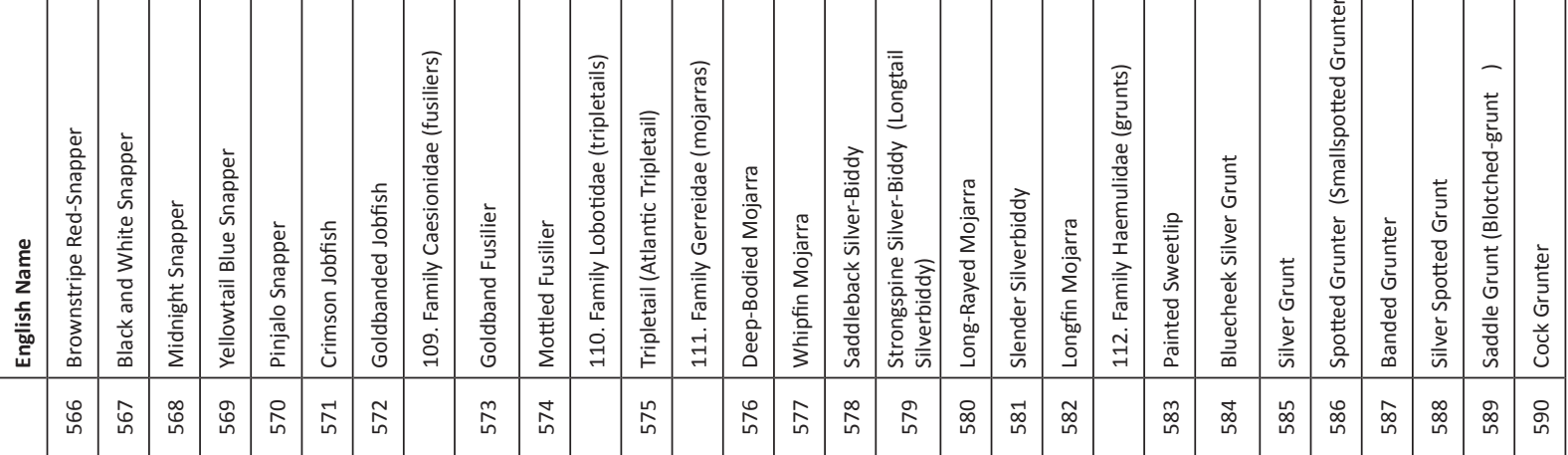




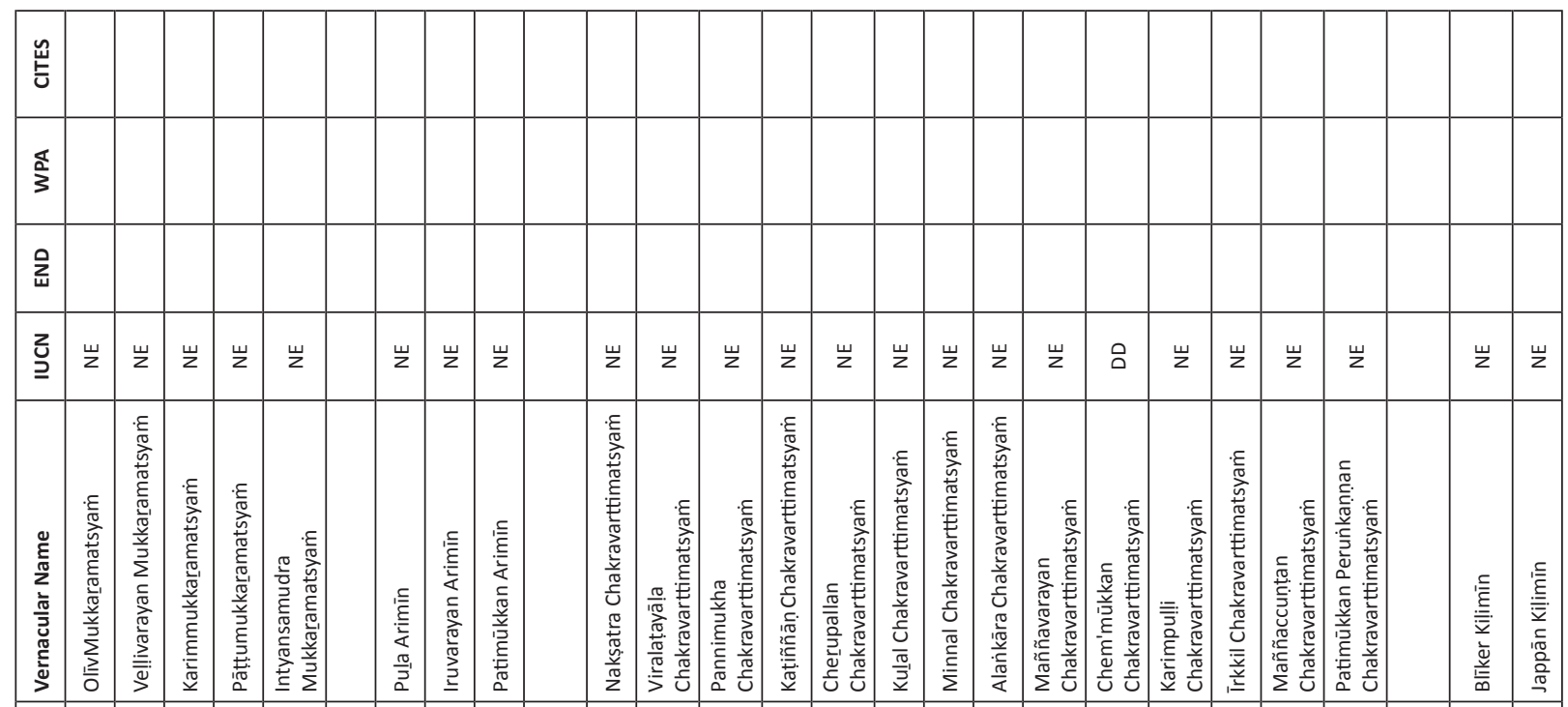

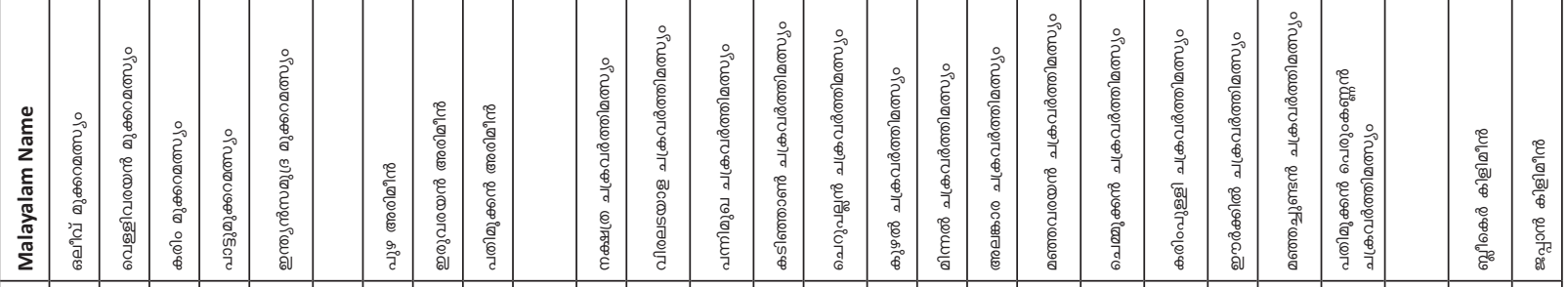

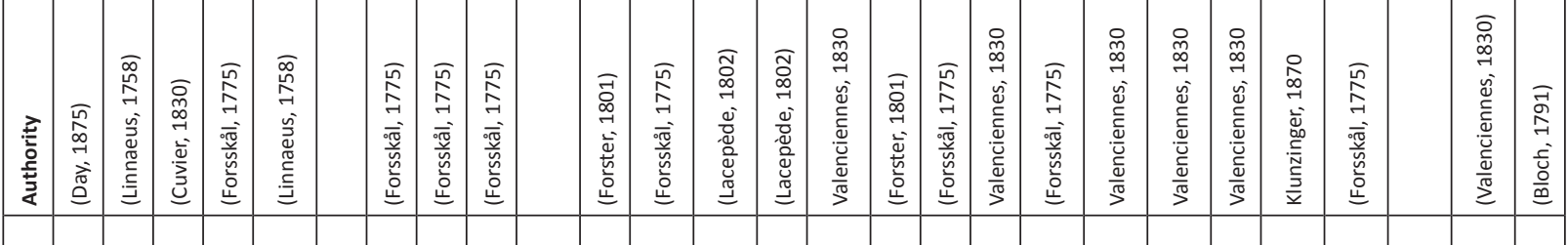

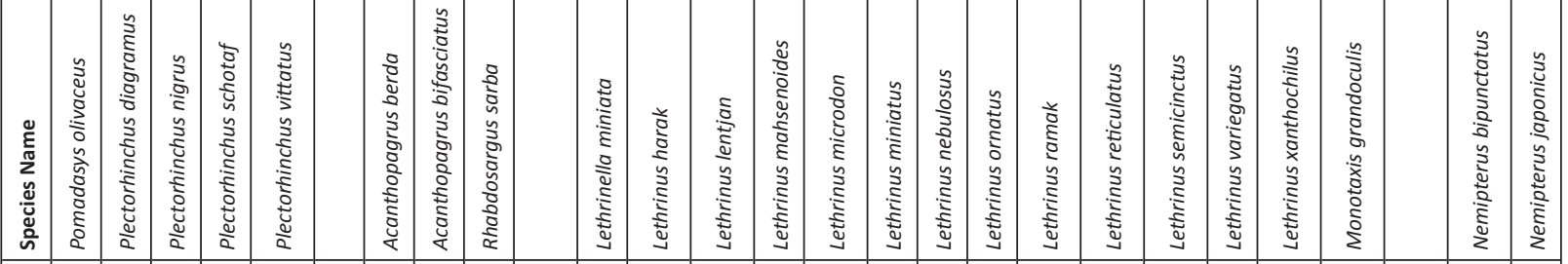

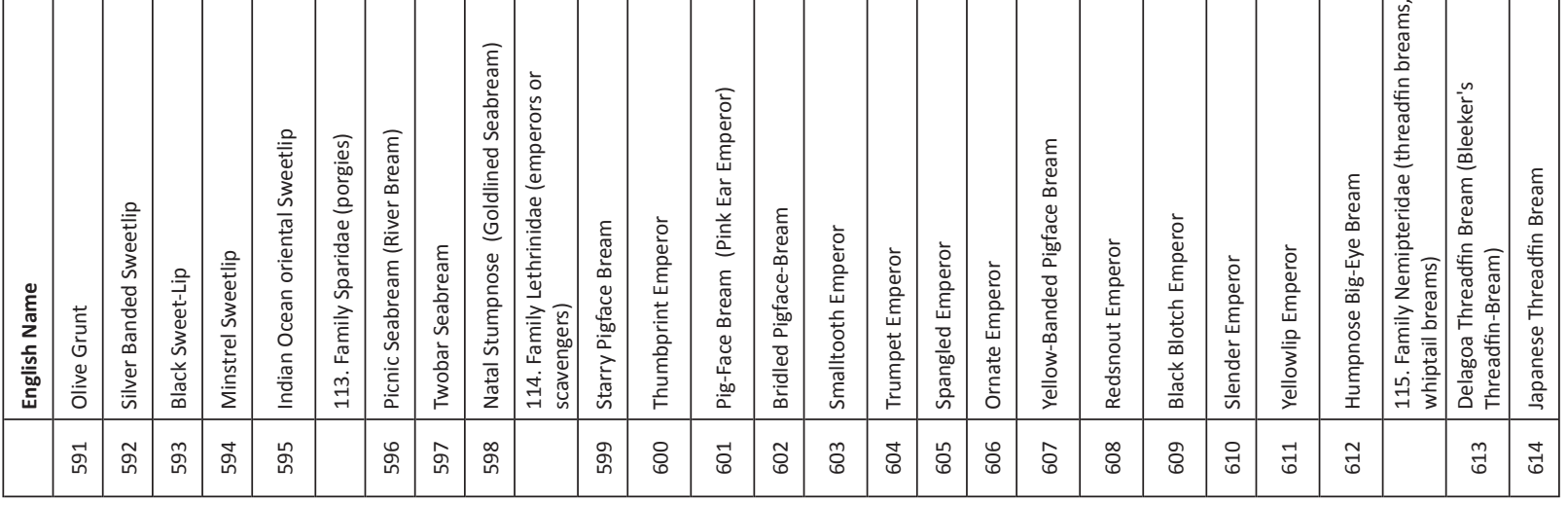




\begin{tabular}{|c|c|c|c|c|c|c|c|c|c|c|c|c|c|c|c|c|c|c|c|c|c|c|c|c|c|c|c|c|}
\hline 峞 & & & & & & & & & & & & & & & & & & & & & & & & & & & & \\
\hline$\hat{\jmath}^{\alpha}$ & & & & & & & & & & & & & & & & & & & & & & & & & & & & \\
\hline$\sum_{\text {I }}^{2}$ & & & & & & & & & & & & & & & & & & & & & & & & & & & & \\
\hline ż & $\vec{w}$ & 㟧 & 岁 & 㟧 & 岁 & 㟧 & 岁 & 岁 & 岁 & 㟧 & 山्山 & 山्山 & w & 㟧 & $\vec{w}$ & 崩 & 崖 & 㟧 & 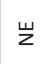 & 岁 & 岁 & 崩 & 岁 & 岁 & $\overrightarrow{\mathrm{z}}$ & 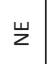 & $\vec{z}$ & 岁 \\
\hline 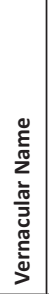 & 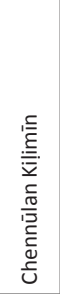 & 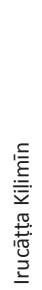 & 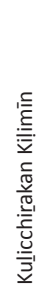 & 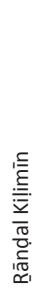 & 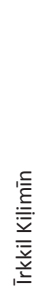 & 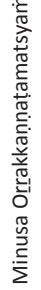 & 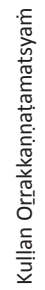 & 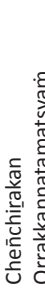 & 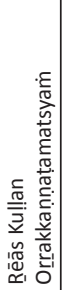 & 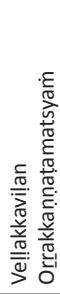 & 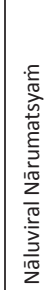 & 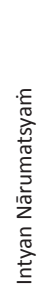 & 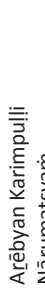 & 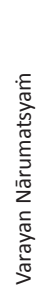 & 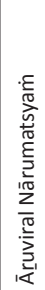 & 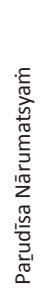 & 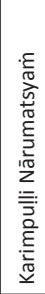 & 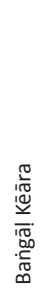 & 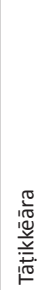 & 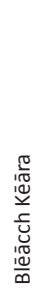 & 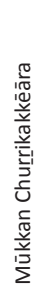 & 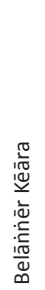 & 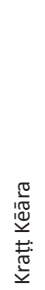 & 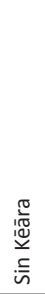 & 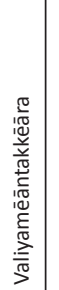 & 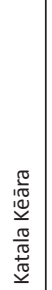 & 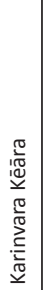 & 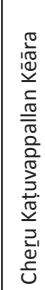 \\
\hline
\end{tabular}

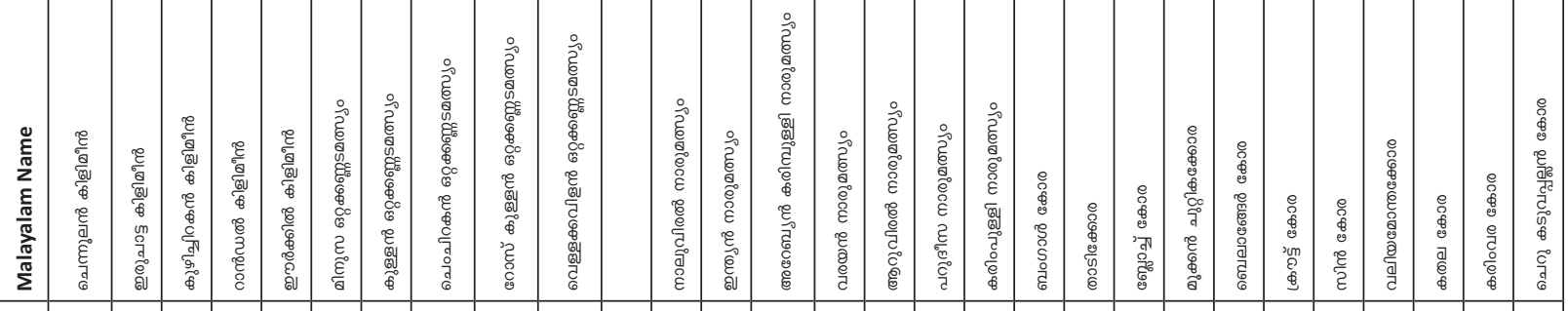

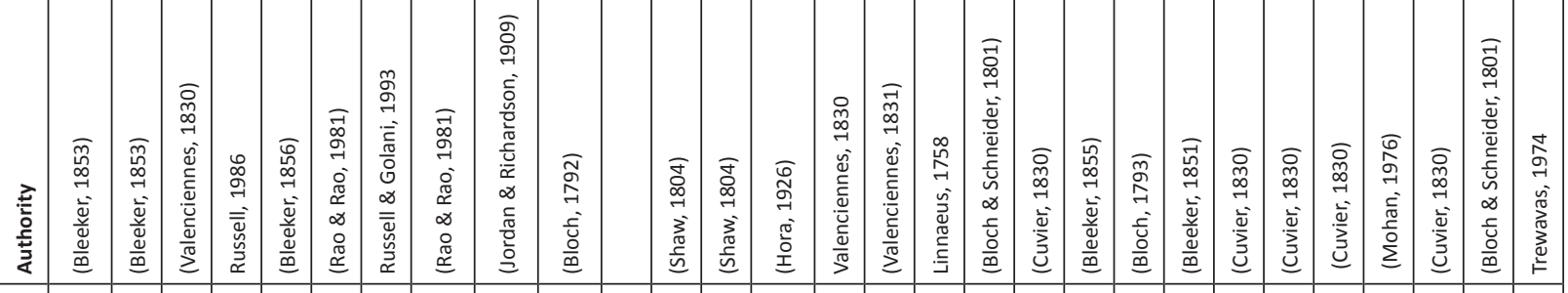

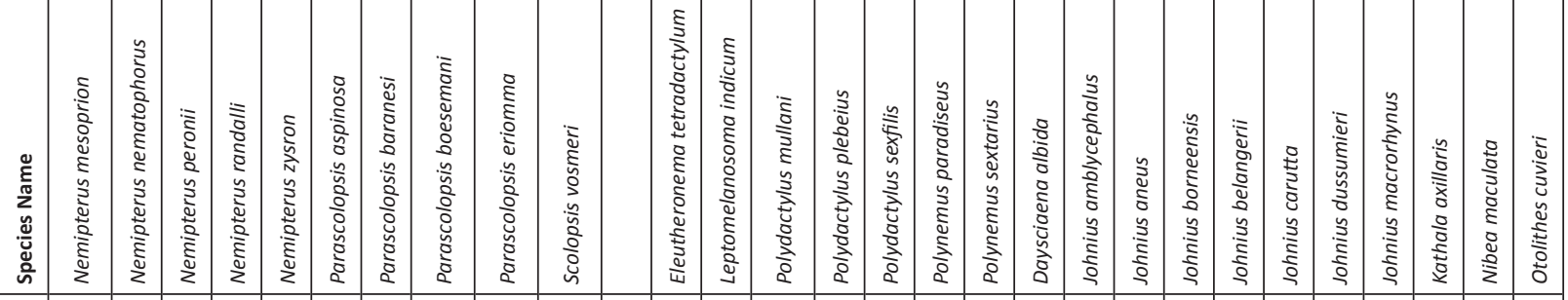

\begin{tabular}{|c|c|c|c|c|c|c|c|c|c|c|c|c|c|c|c|c|c|c|c|c|c|c|c|}
\hline 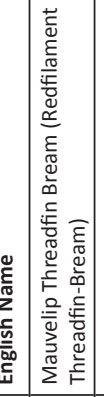 & 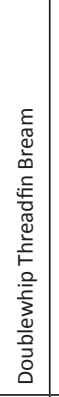 & 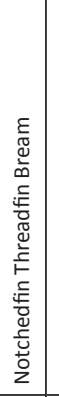 & 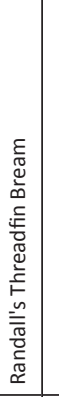 & 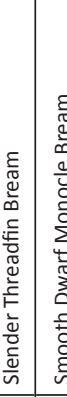 & 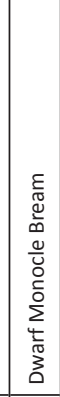 & 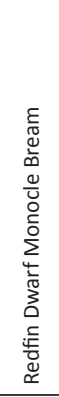 & 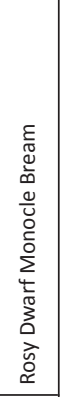 & 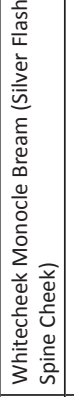 & 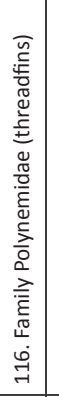 & 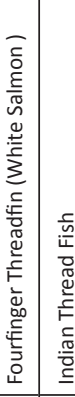 & 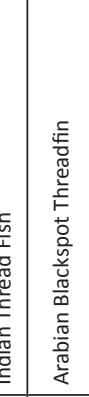 & 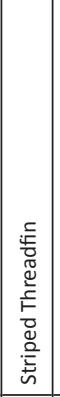 & 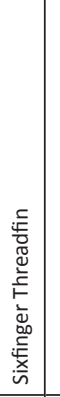 & 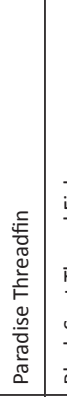 & 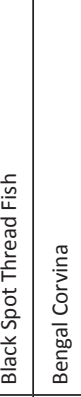 & 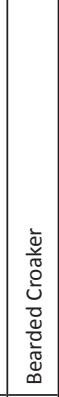 & 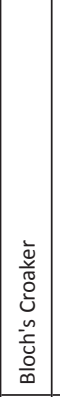 & 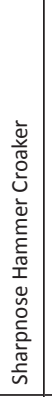 & 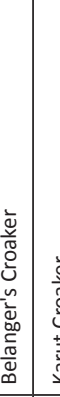 & 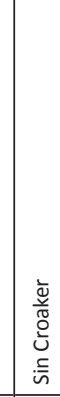 & 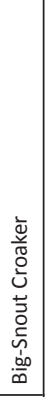 & 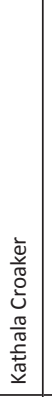 & 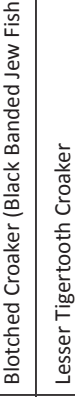 \\
\hline 2 & 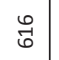 & $\hat{0}$ & $\begin{array}{c}\stackrel{\infty}{0} \\
\vec{b}\end{array}$ & ఫे & تิ & $\widetilde{\Xi}$ & ఇ్ర & ఫ్ర & & $\underset{\sigma}{0}$ & \begin{tabular}{l|l}
\multirow{\sigma}{0}{} & $\widehat{\sigma}$
\end{tabular} & 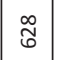 & ฉ్రి & : & \begin{tabular}{l|l}
$\overrightarrow{0}$ & $\tilde{0}$
\end{tabular} & 艿 & 苟 & مِ & 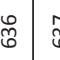 & & ஜूठ & f & \\
\hline
\end{tabular}




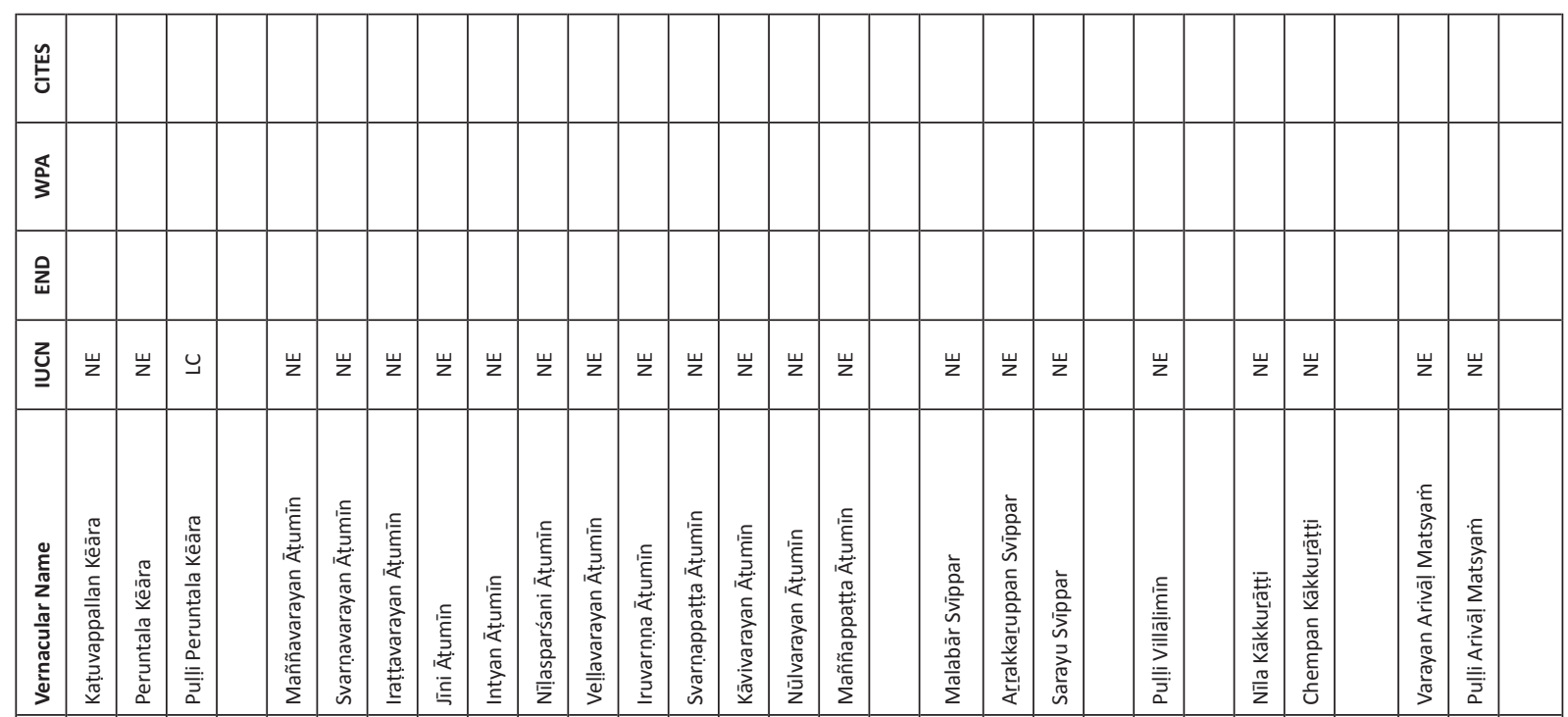

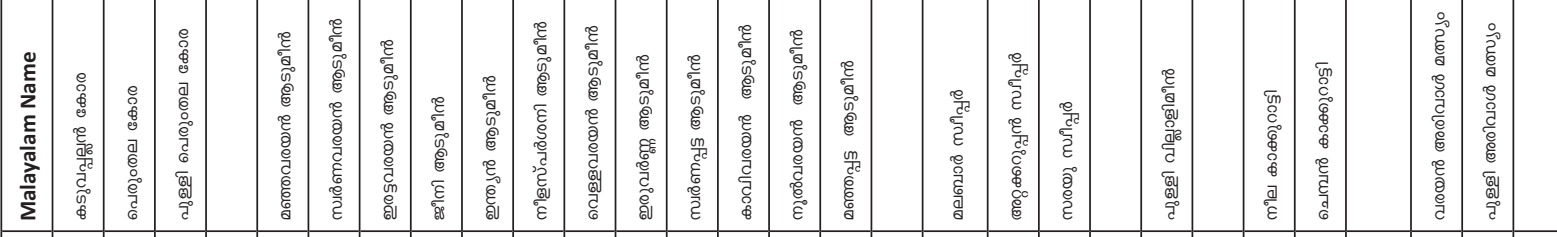

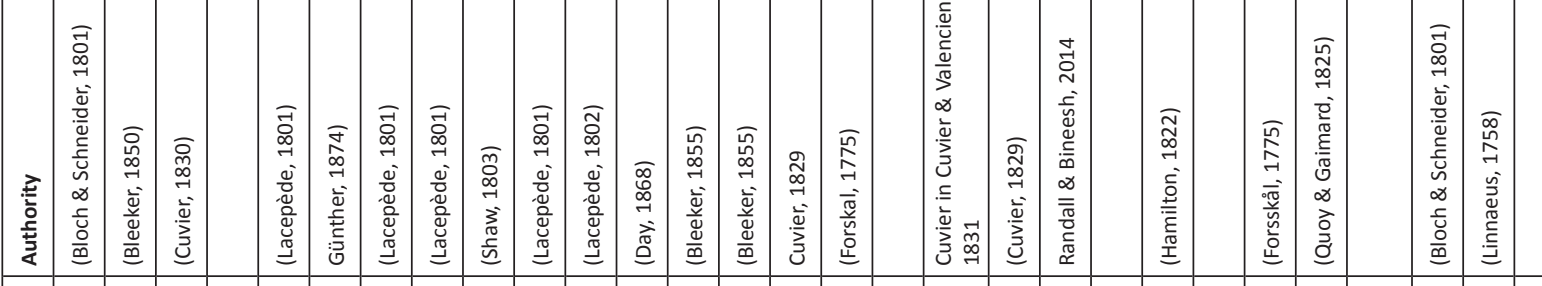

\begin{tabular}{|c|c|c|c|c|c|c|c|c|c|c|c|c|c|c|c|c|c|c|c|c|c|c|c|c|c|c|}
\hline 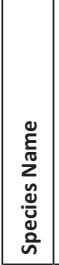 & 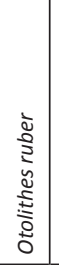 & 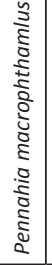 & 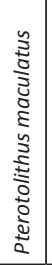 & & 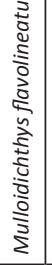 & 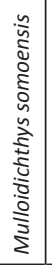 & 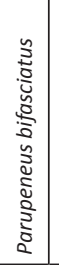 & 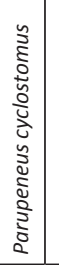 & 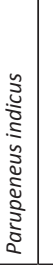 & 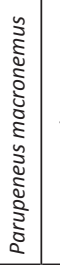 & 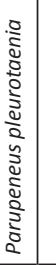 & 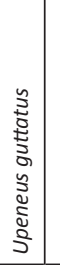 & 芯 & 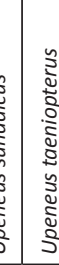 & 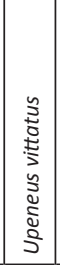 & & 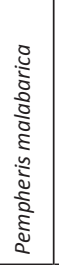 & 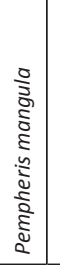 & 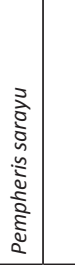 & 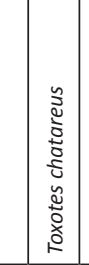 & & 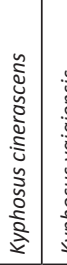 & & 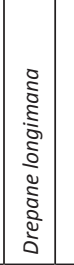 & 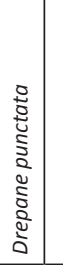 & \\
\hline 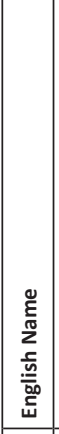 & 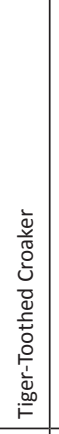 & 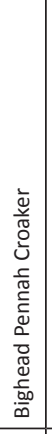 & 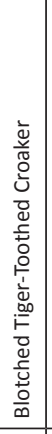 & 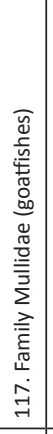 & 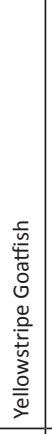 & 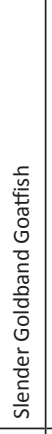 & 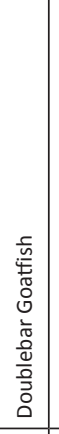 & 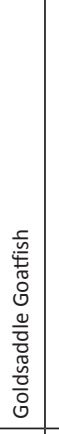 & 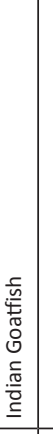 & 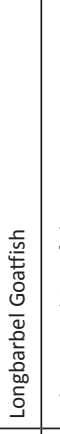 & 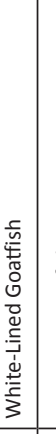 & 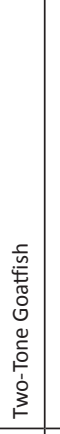 & 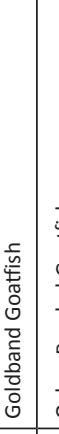 & 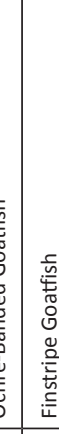 & 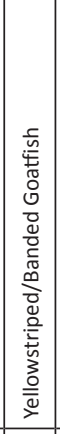 & 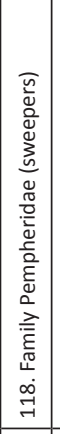 & 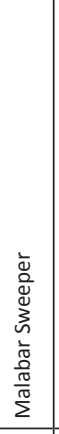 & 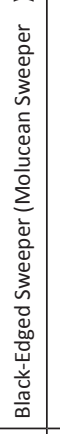 & 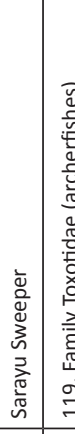 & 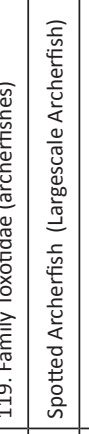 & 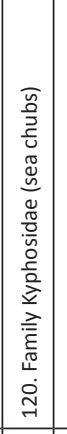 & 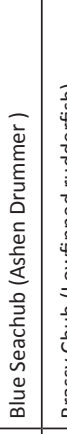 & 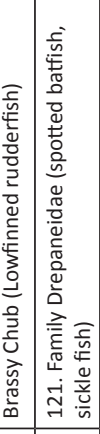 & 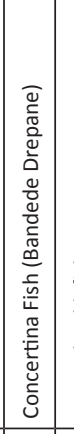 & 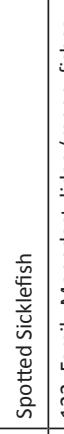 & 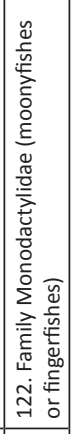 \\
\hline & q & $\begin{array}{l}\text { G } \\
\text {. }\end{array}$ & \&్ & & $\begin{array}{l}0 \\
0\end{array}$ & fे & $\begin{array}{l}\infty \\
\mathbb{b}\end{array}$ & o & $\begin{array}{l}0 \\
\text { 足 }\end{array}$ & $\overrightarrow{. ~}$ & జี & ஜ̂́ి & 苑 & $\begin{array}{l}\hat{0} \\
\end{array}$ & 芯 & & 㗐 & 㽞 & $\stackrel{\circ}{\circ}$ & $\overrightarrow{0}$ & & 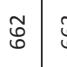 & : & \begin{tabular}{|l|} 
\\
\\
\end{tabular} & 䀫 & \\
\hline
\end{tabular}




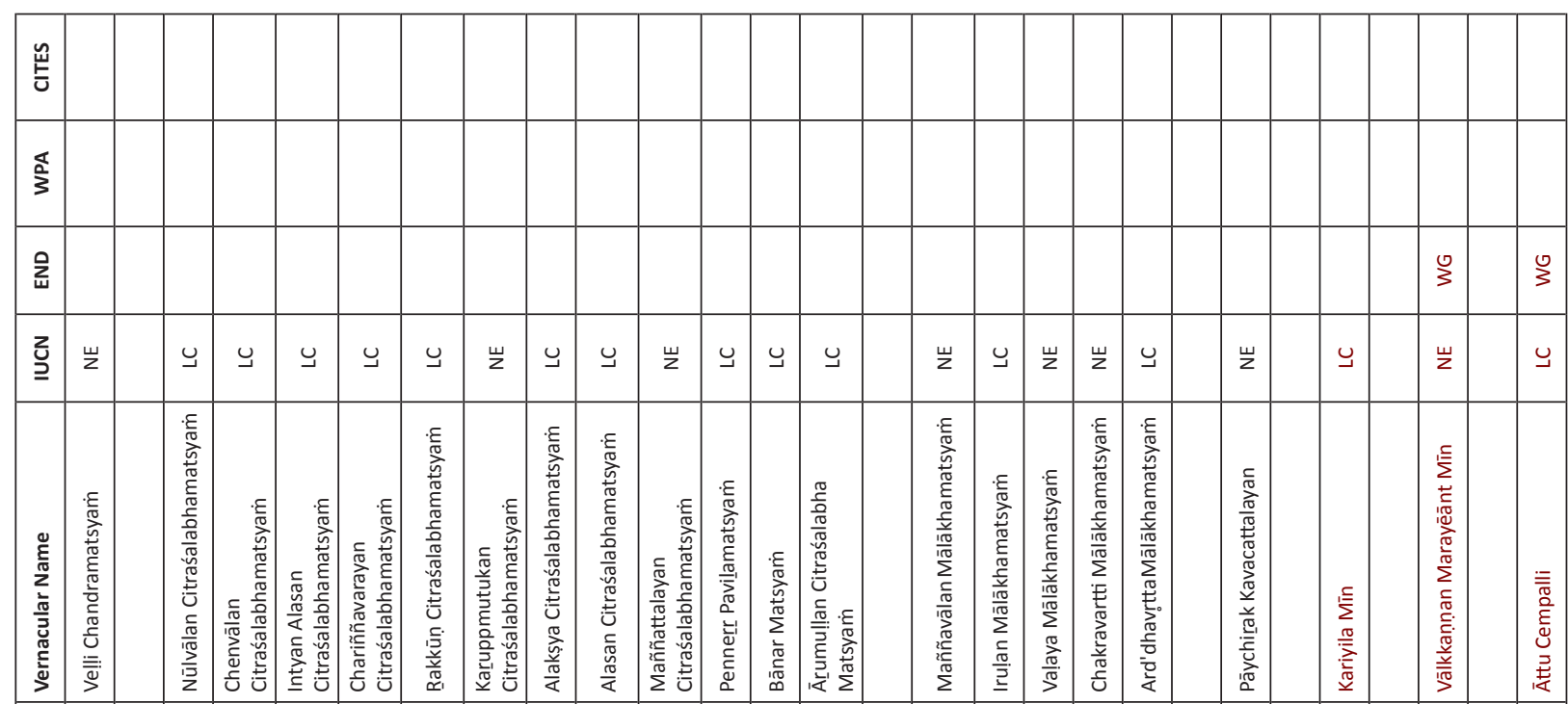

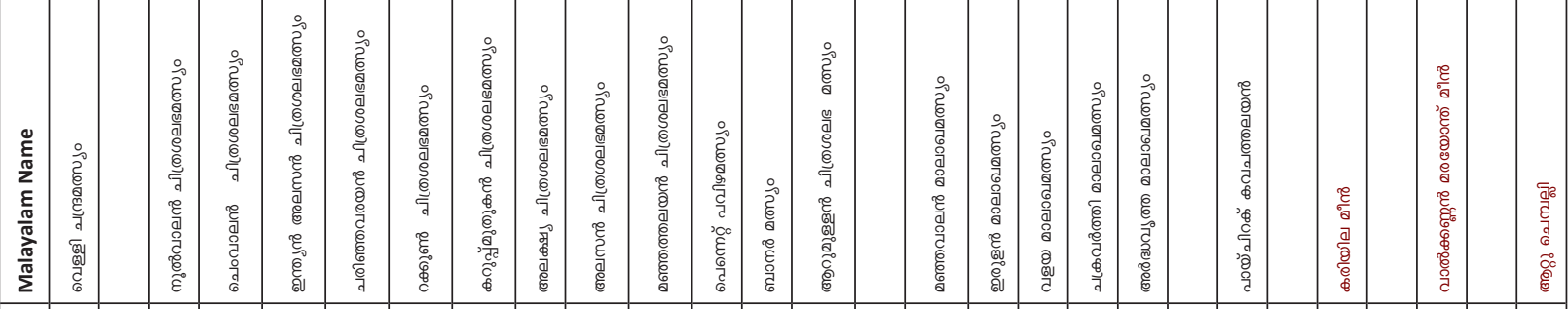

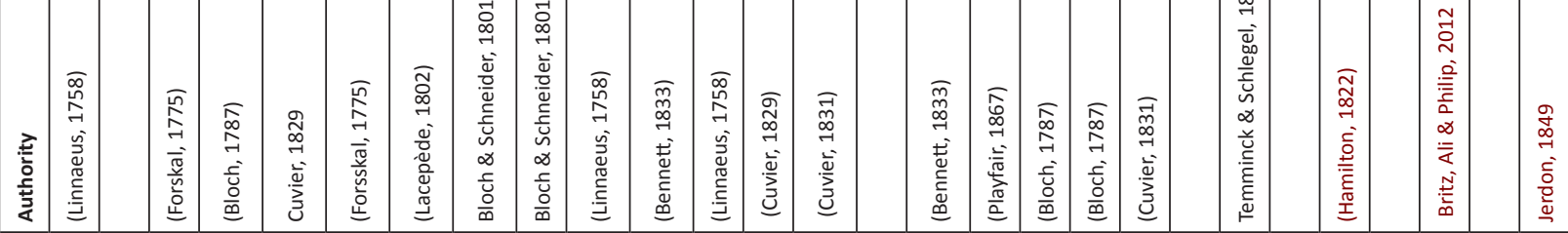

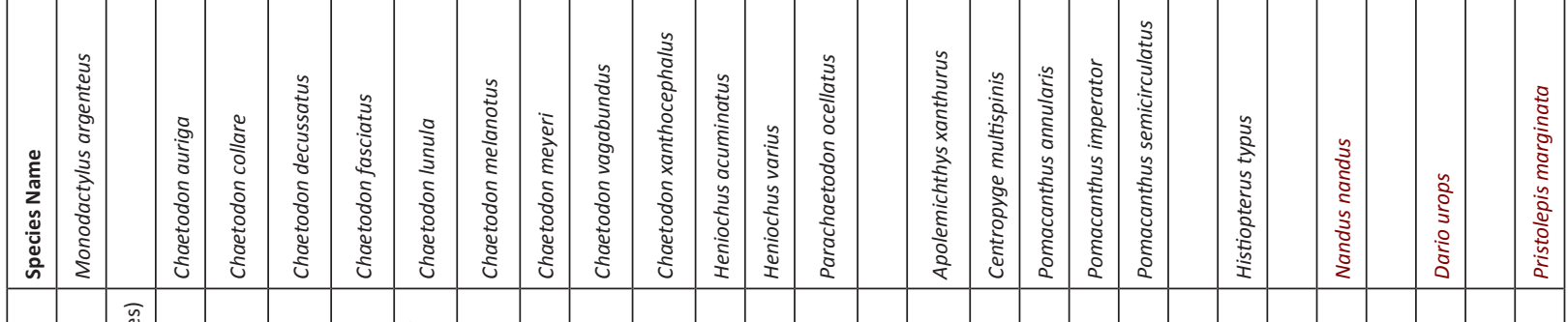

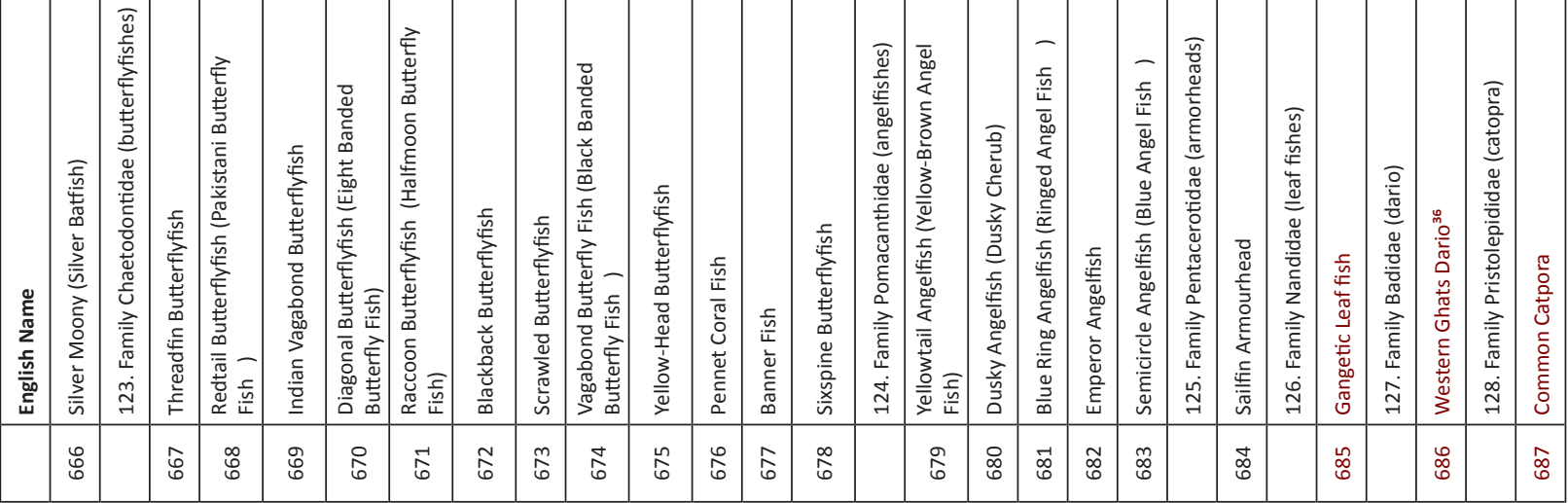




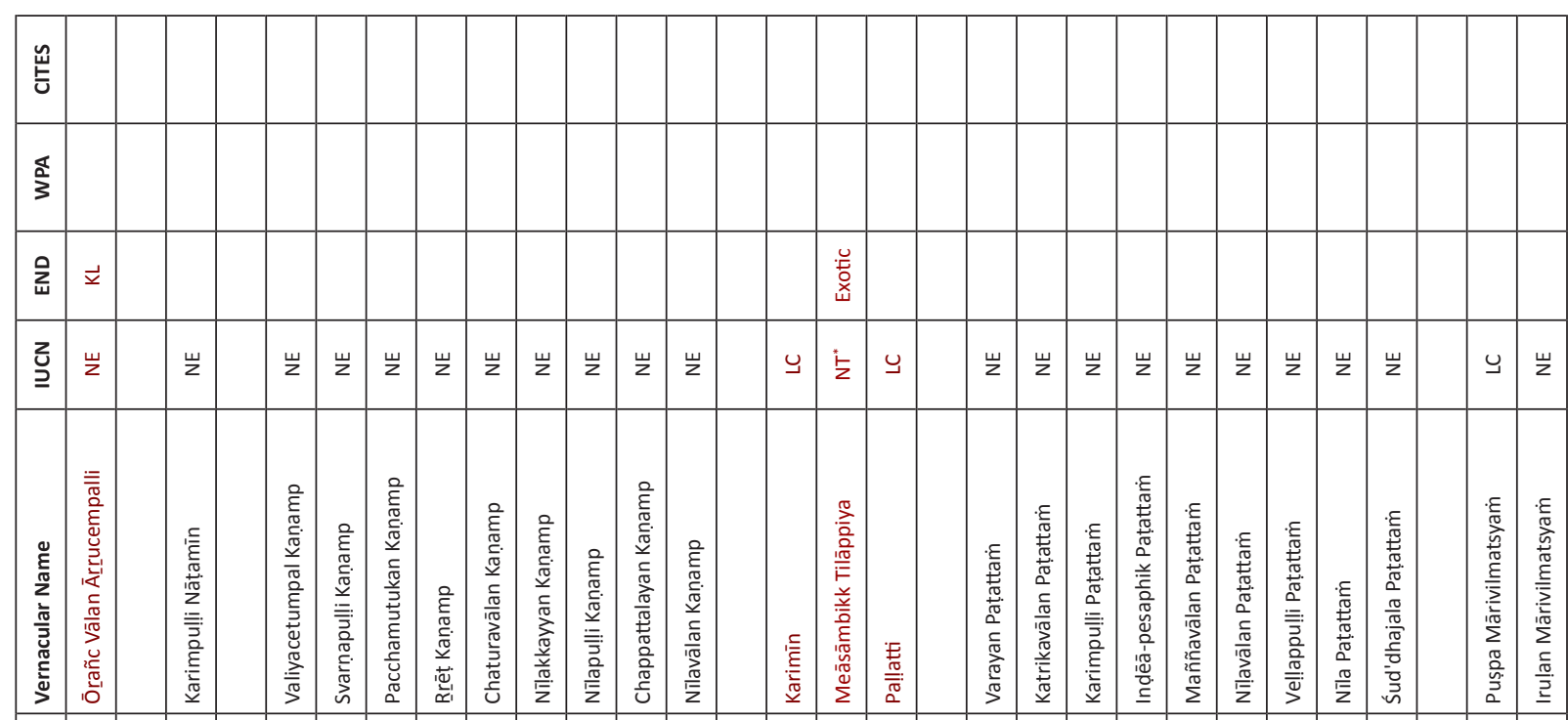

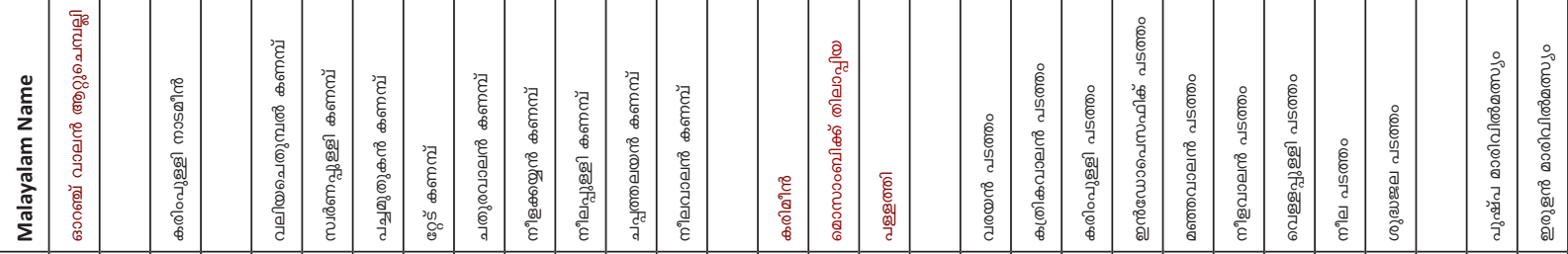

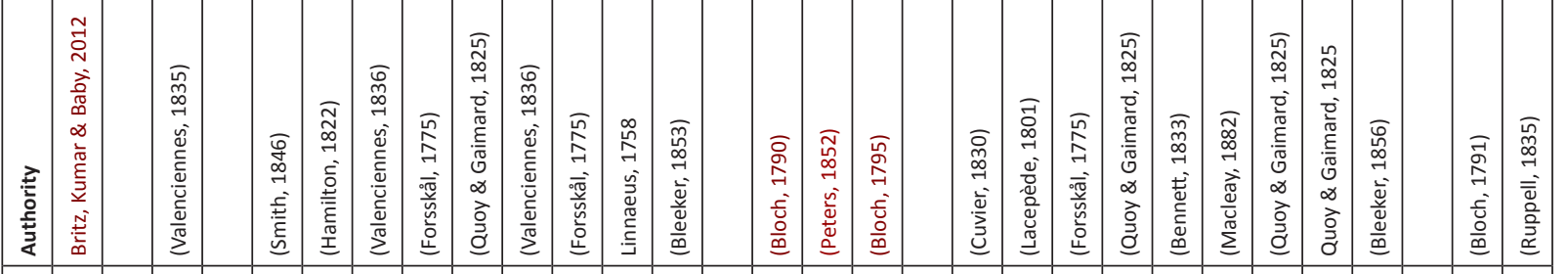

\begin{tabular}{|c|c|c|c|c|c|c|c|c|c|c|c|c|c|c|c|c|c|c|c|c|c|c|c|c|c|c|}
\hline 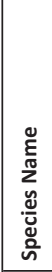 & 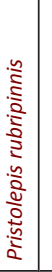 & & 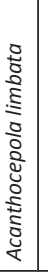 & & 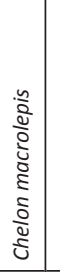 & 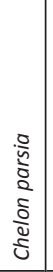 & 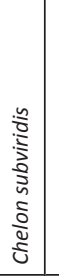 & 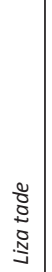 & 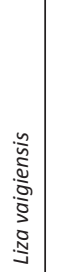 & 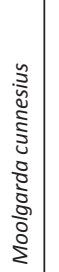 & 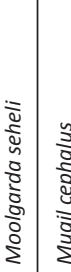 & 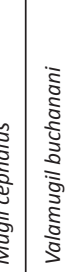 & & 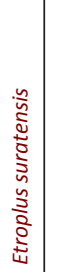 & 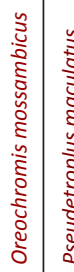 & 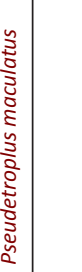 & 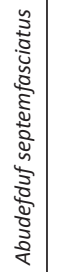 & 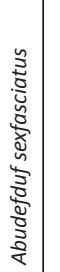 & 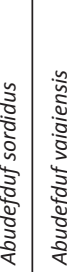 & 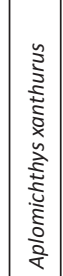 & 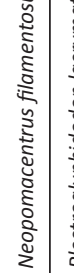 & 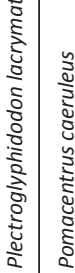 & 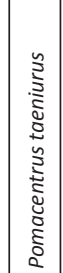 & & 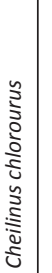 & 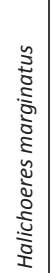 \\
\hline & 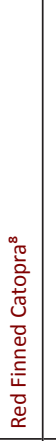 & 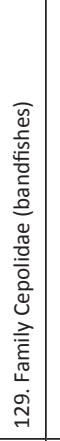 & 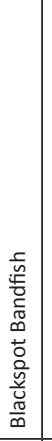 & 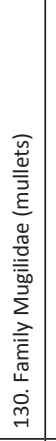 & 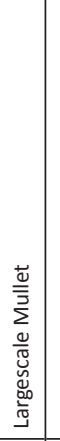 & 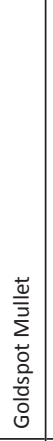 & 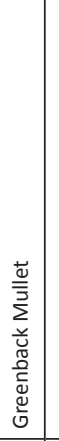 & 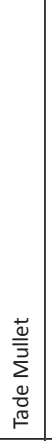 & 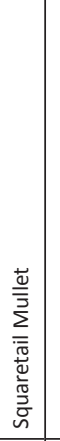 & 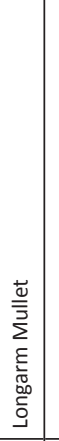 & 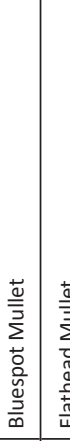 & 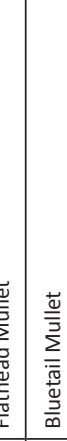 & 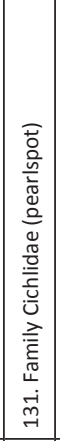 & 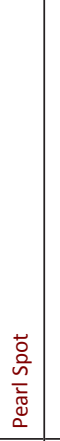 & 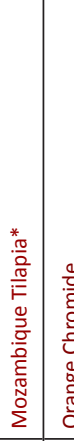 & 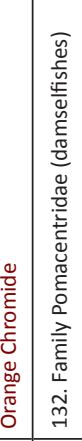 & 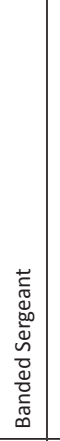 & 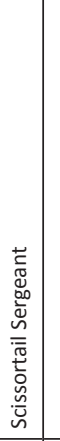 & 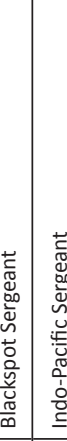 & 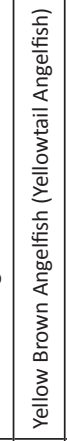 & 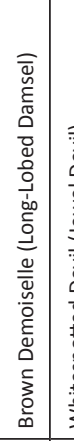 & 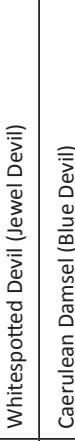 & 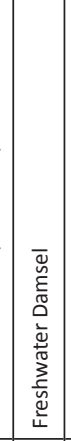 & 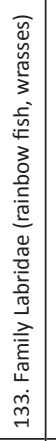 & 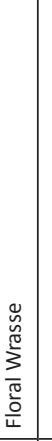 & 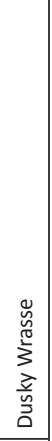 \\
\hline & \begin{tabular}{l}
$\infty$ \\
$\infty$ \\
\hdashline
\end{tabular} & & ఖ్రి & & : & $\overrightarrow{0}$ & ฮే & 茴 & 志 & 员 & $\begin{array}{l}\circ \\
0\end{array}$ & Љ్రి & & g. & 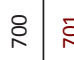 & 5 & $\stackrel{2}{\curvearrowright}$ & $\stackrel{m}{\stackrel{2}{2}}$ & 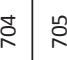 & 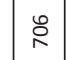 & $\hat{\imath}$ & $\stackrel{\infty}{尺}$ & 임 & & $\vec{\nabla}$ & กี \\
\hline
\end{tabular}




\begin{tabular}{|c|c|c|c|c|c|c|c|c|c|c|c|c|c|c|c|c|c|c|c|c|c|c|c|c|}
\hline 岂 & & & & & & & & & & & & & & & & & & & & & & & & \\
\hline$\varliminf_{3}^{\alpha}$ & & & & & & & & & & & & & & & & & & & & & & & & \\
\hline$\overbrace{u}^{\rho}$ & & & & & & & & & & & & & & & & & & & & & & & & \\
\hline zu & $\breve{~}$ & u & u & $\underline{y}$ & ฉ & u & y & y & צ & u & u & u & y & 崩 & 㟧 & 崩 & 㟧 & 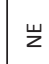 & 亗 & 亗 & $\vec{z}$ & 岂 & 岁 & $\underline{\mathrm{z}}$ \\
\hline 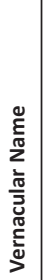 & 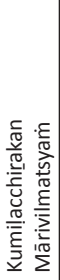 & 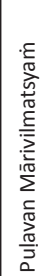 & 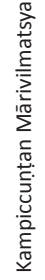 & 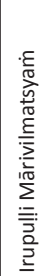 & 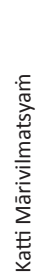 & 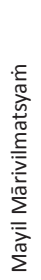 & 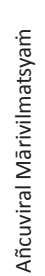 & 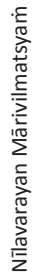 & 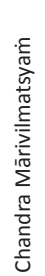 & 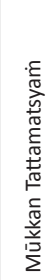 & 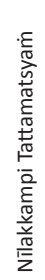 & 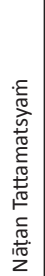 & 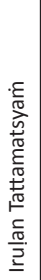 & 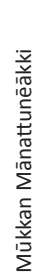 & 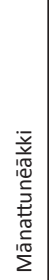 & 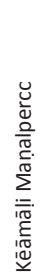 & 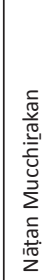 & 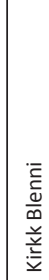 & 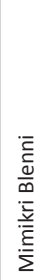 & 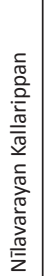 & 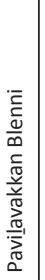 & 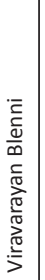 & 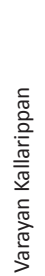 & 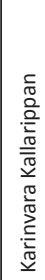 \\
\hline
\end{tabular}

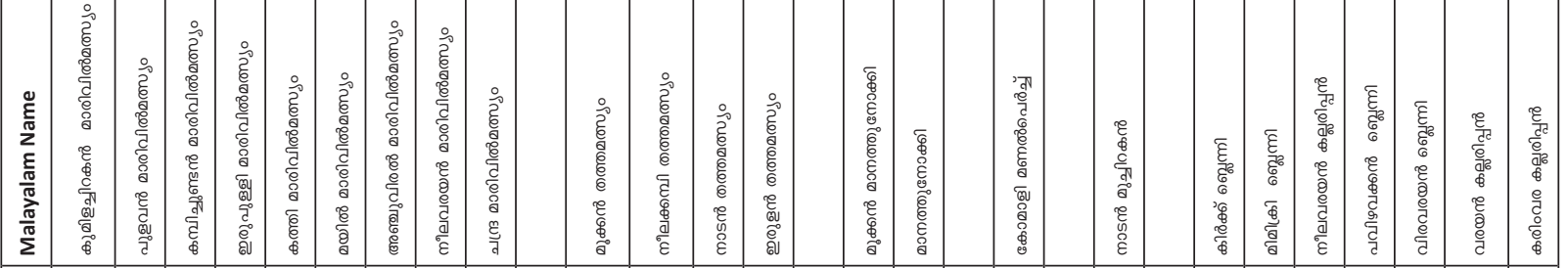

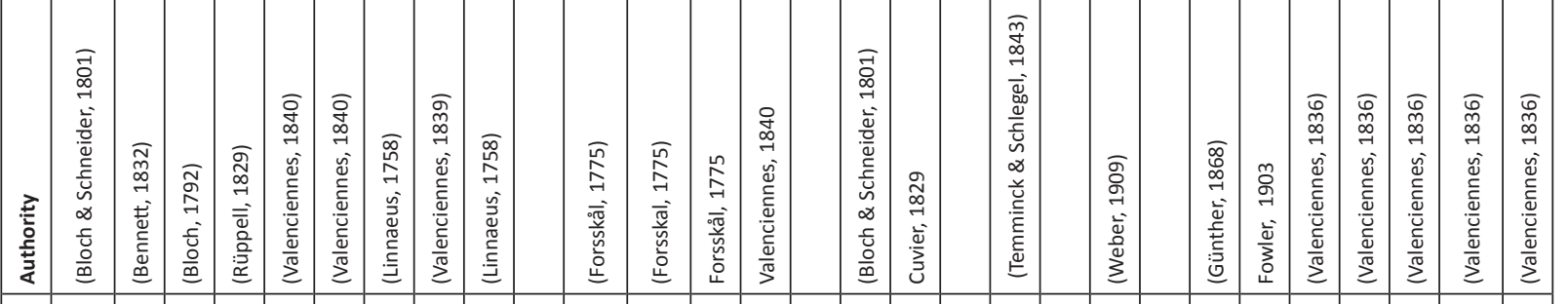

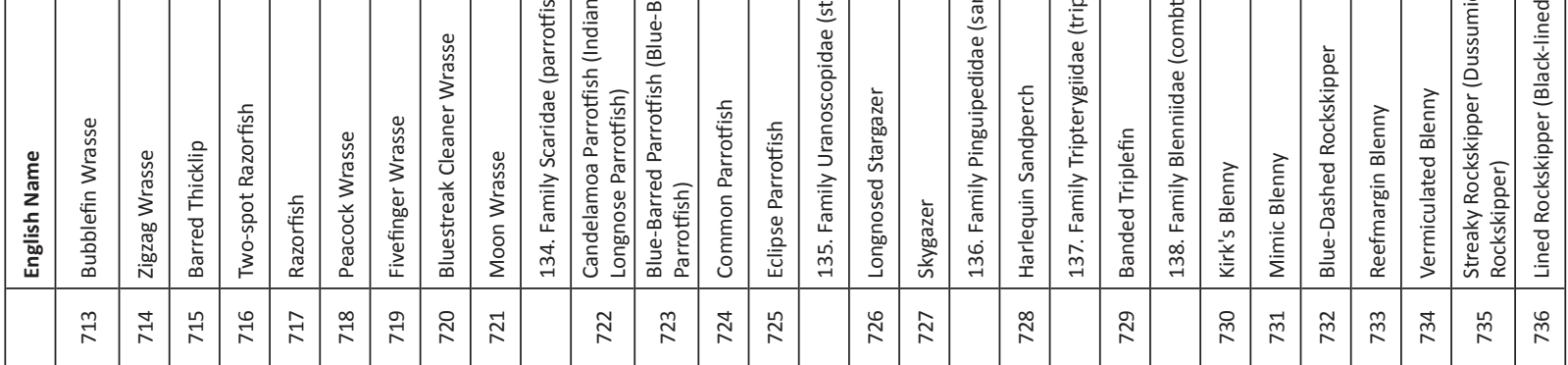




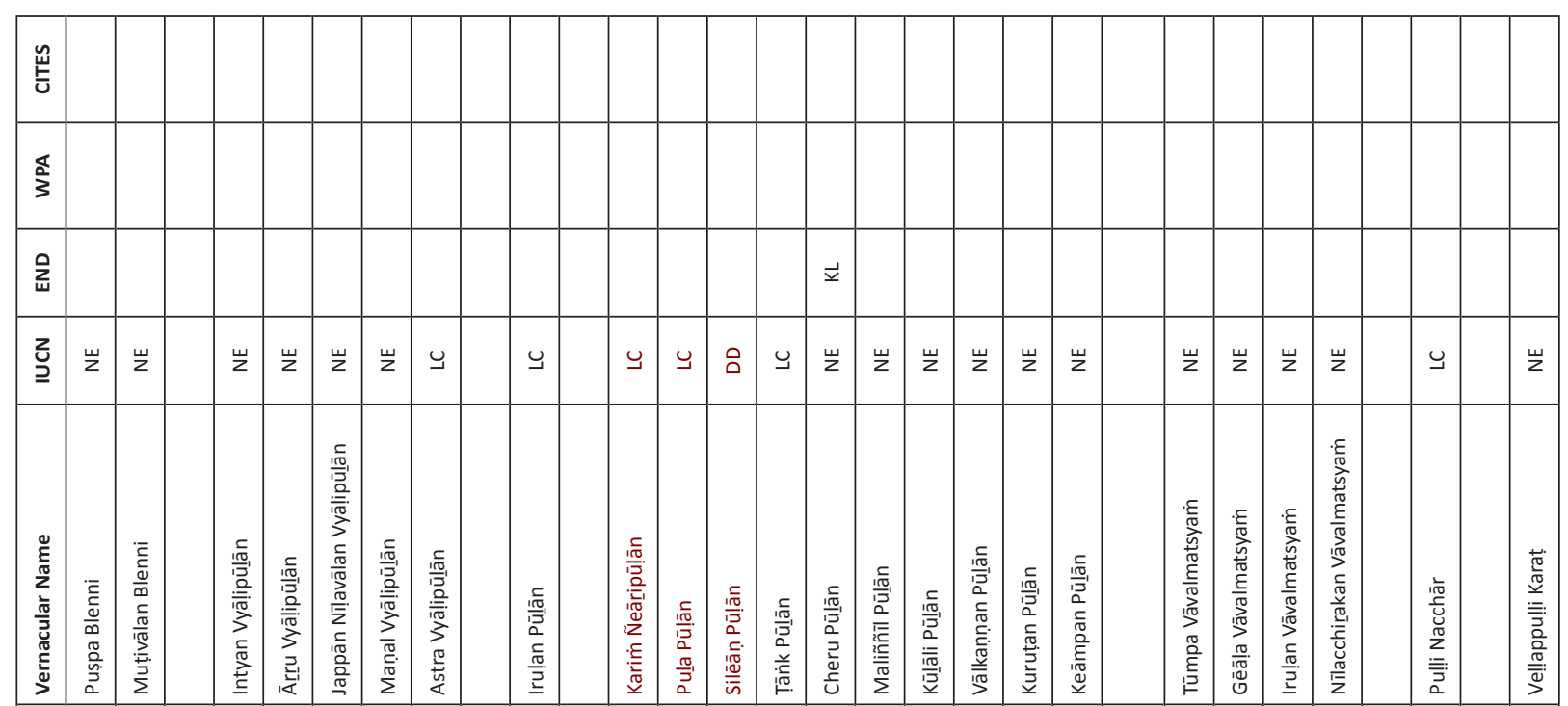

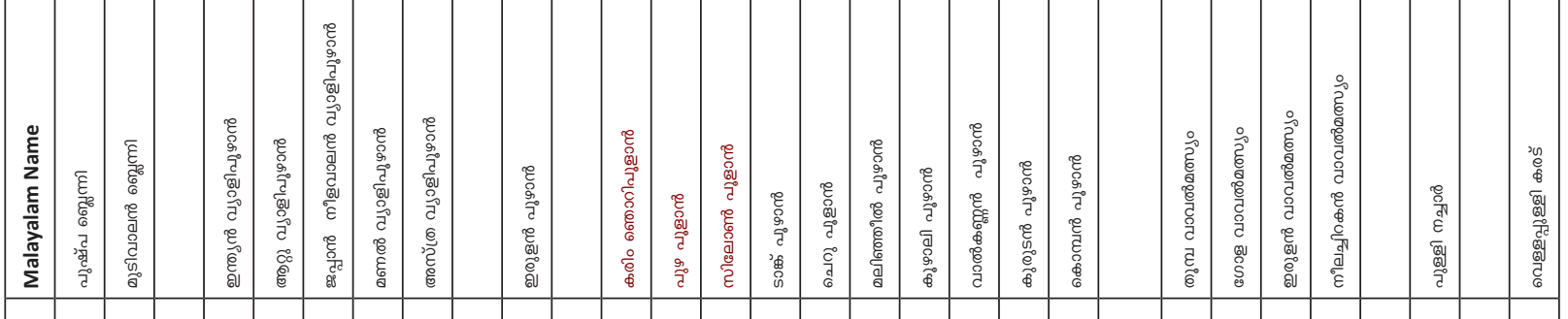

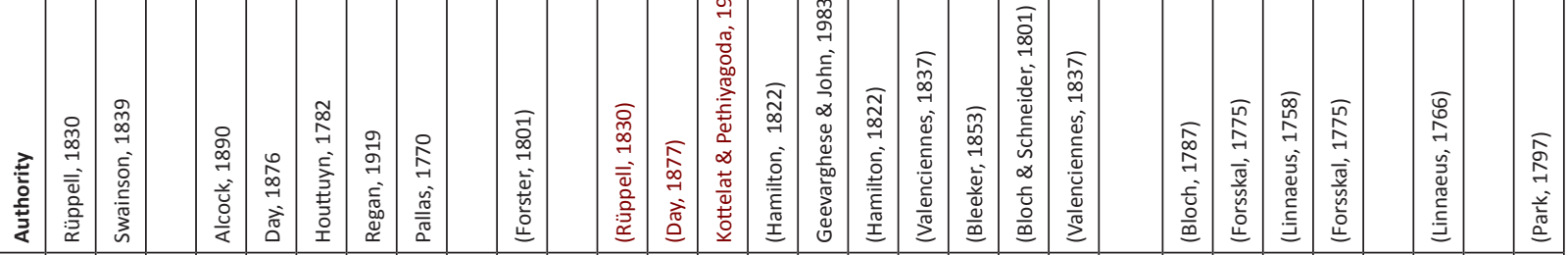

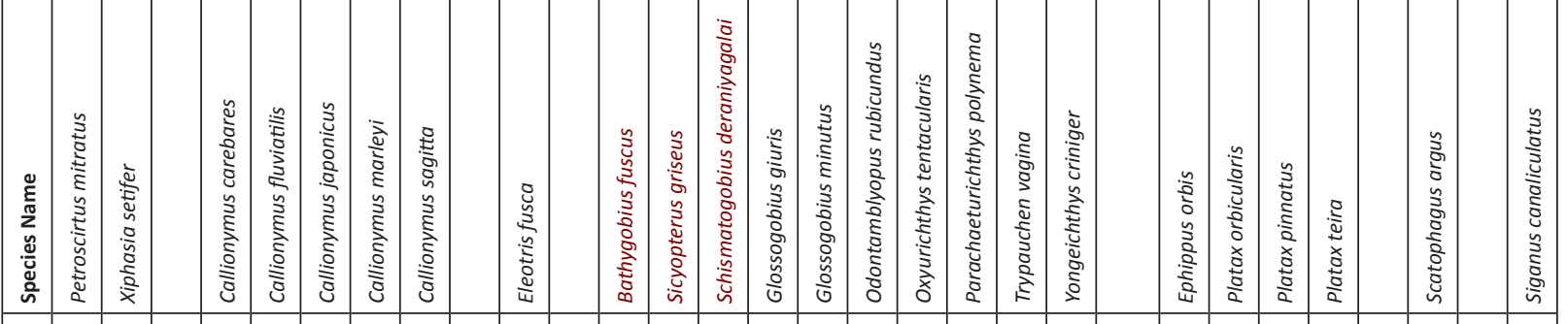

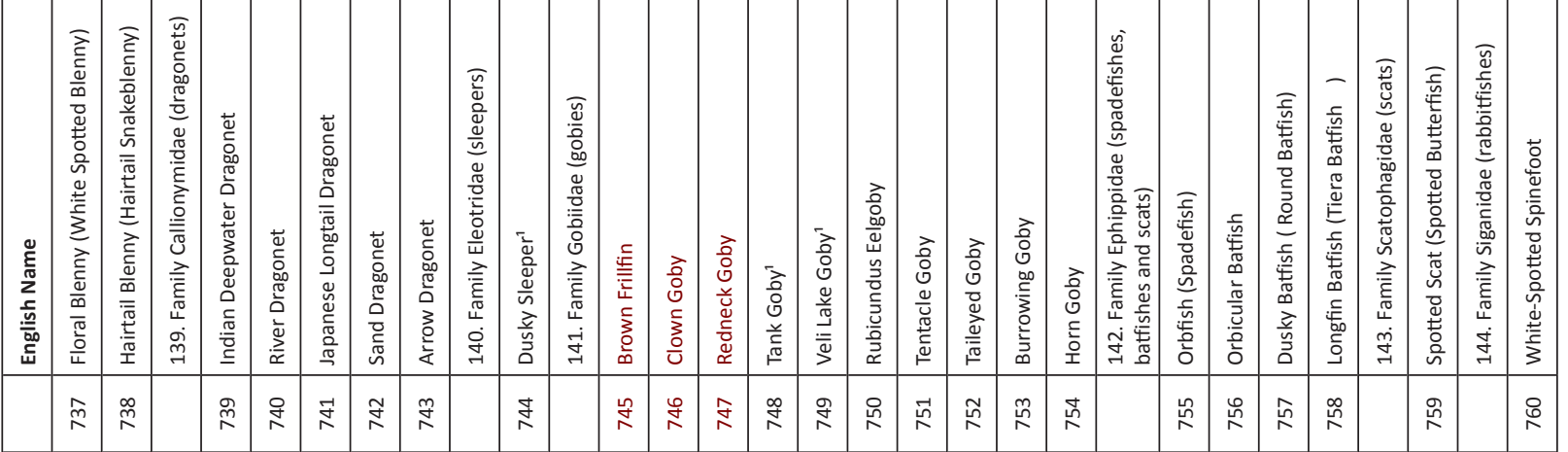




\begin{tabular}{|c|c|c|c|c|c|c|c|c|c|c|c|c|c|c|c|c|c|c|c|c|c|c|c|c|c|}
\hline 岂 & & & & & & & & & & & & & & & & & & & & & & & & & \\
\hline$\frac{a}{3}$ & & & & & & & & & & & & & & & & & & & & & & & & & \\
\hline 总 & & & & & & & & & & & & & & & & & & & & & & & & & \\
\hline zu & 亗 & 崩 & 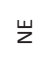 & एu & $\frac{u}{z}$ & $\frac{\mathrm{u}}{z}$ & צ & u & y & צ & y & צ & 崩 & u & 崩 & 崩 & 崩 & 岁 & 岂 & 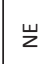 & 岁 & 崩 & 岂 & $\underline{\underline{z}}$ & 岁 \\
\hline 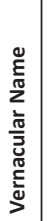 & 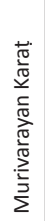 & 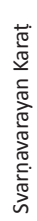 & 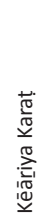 & 蒂 & 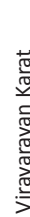 & 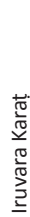 & 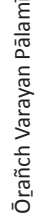 & 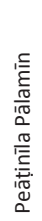 & 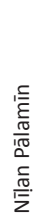 & 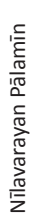 & 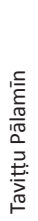 & 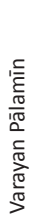 & 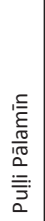 & 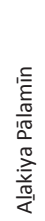 & 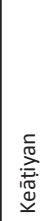 & 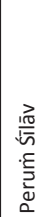 & 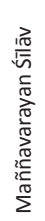 & 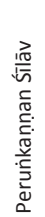 & 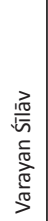 & 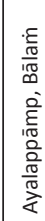 & 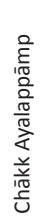 & 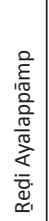 & 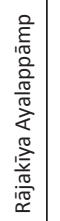 & 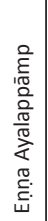 & 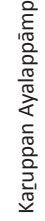 \\
\hline
\end{tabular}

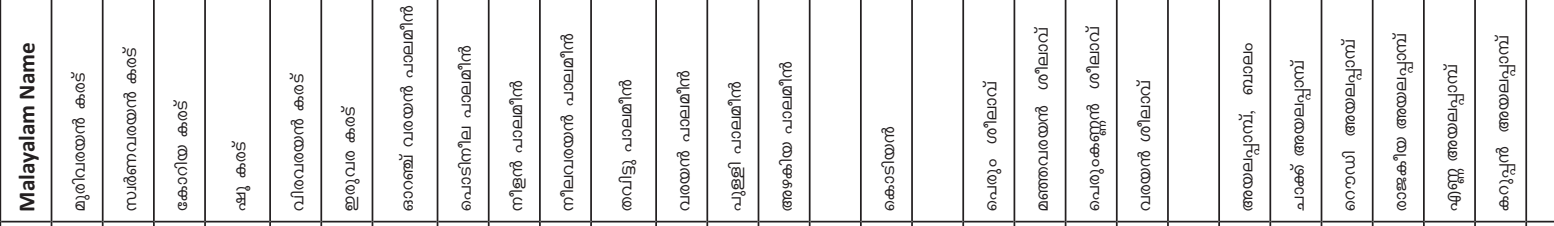

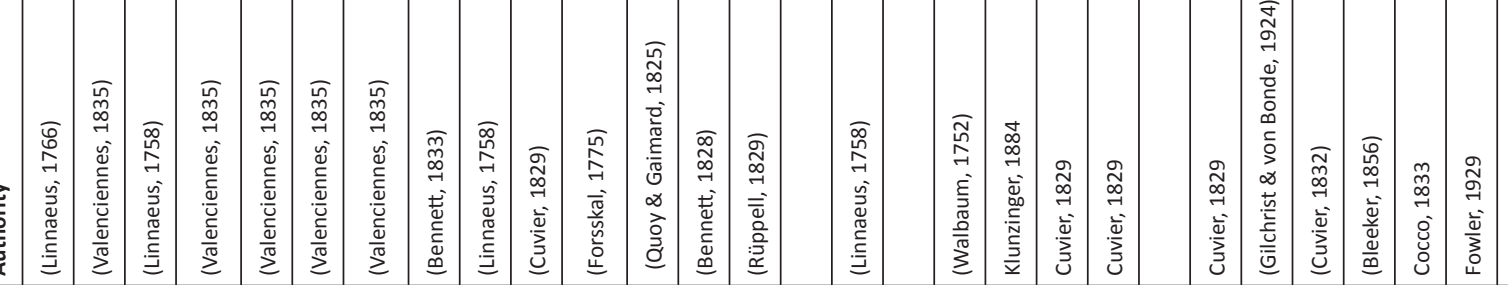

\begin{tabular}{|c|c|c|c|c|c|c|c|c|c|c|c|c|c|c|c|c|c|c|c|c|c|c|c|c|c|}
\hline & 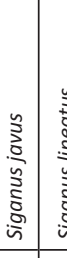 & 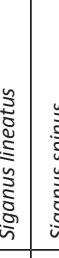 & 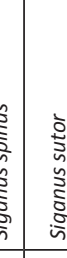 & 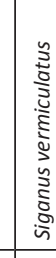 & 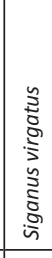 & 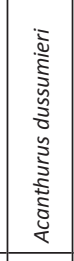 & 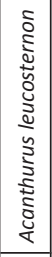 & 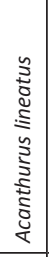 & 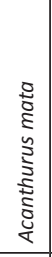 & 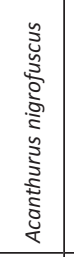 & 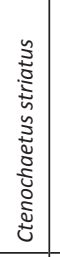 & 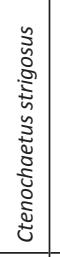 & 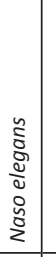 & & 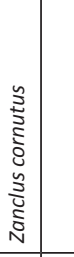 & 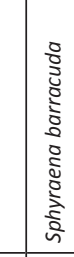 & 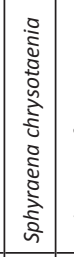 & & & 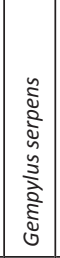 & 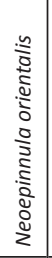 & 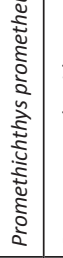 & & & \\
\hline & 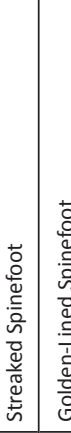 & 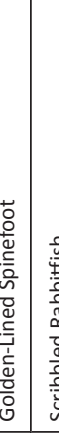 & 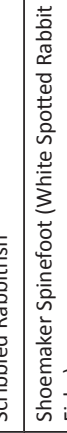 & 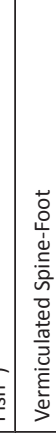 & 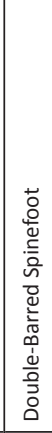 & 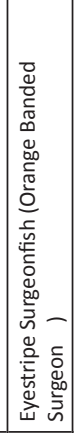 & 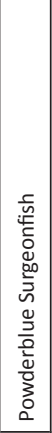 & 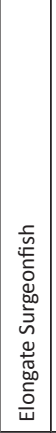 & 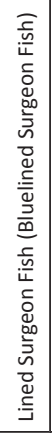 & 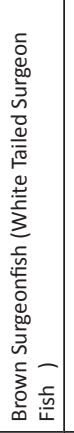 & 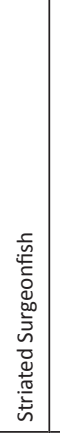 & 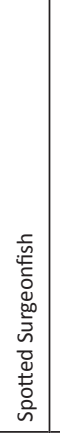 & 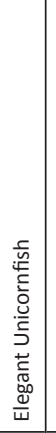 & 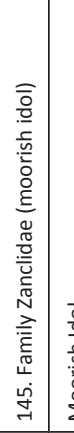 & 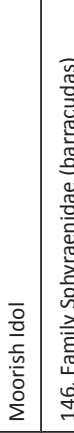 & 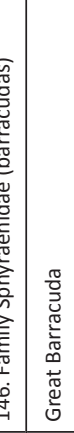 & 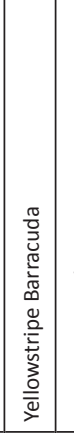 & $\begin{array}{l} \\
\\
\\
0\end{array}$ & 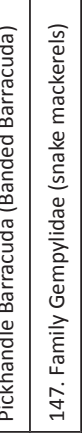 & 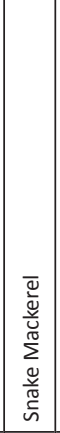 & $\begin{array}{l} \\
\frac{\bar{n}}{\tilde{n}} \\
\frac{\mathbf{v}}{\tilde{n}} \\
\tilde{n}\end{array}$ & 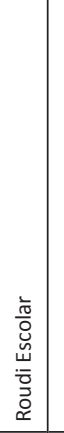 & & & \\
\hline & $\vec{\Phi}$ & శ్ & 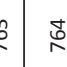 & & & $\grave{\wp}$ & $\begin{array}{l}\infty \\
\stackrel{\infty}{\cap}\end{array}$ & $\pi$ & & & $R$ & 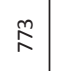 & 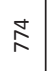 & & & $i$ & $E$ & & & $\stackrel{\infty}{\curvearrowright}$ & & & & & \\
\hline
\end{tabular}




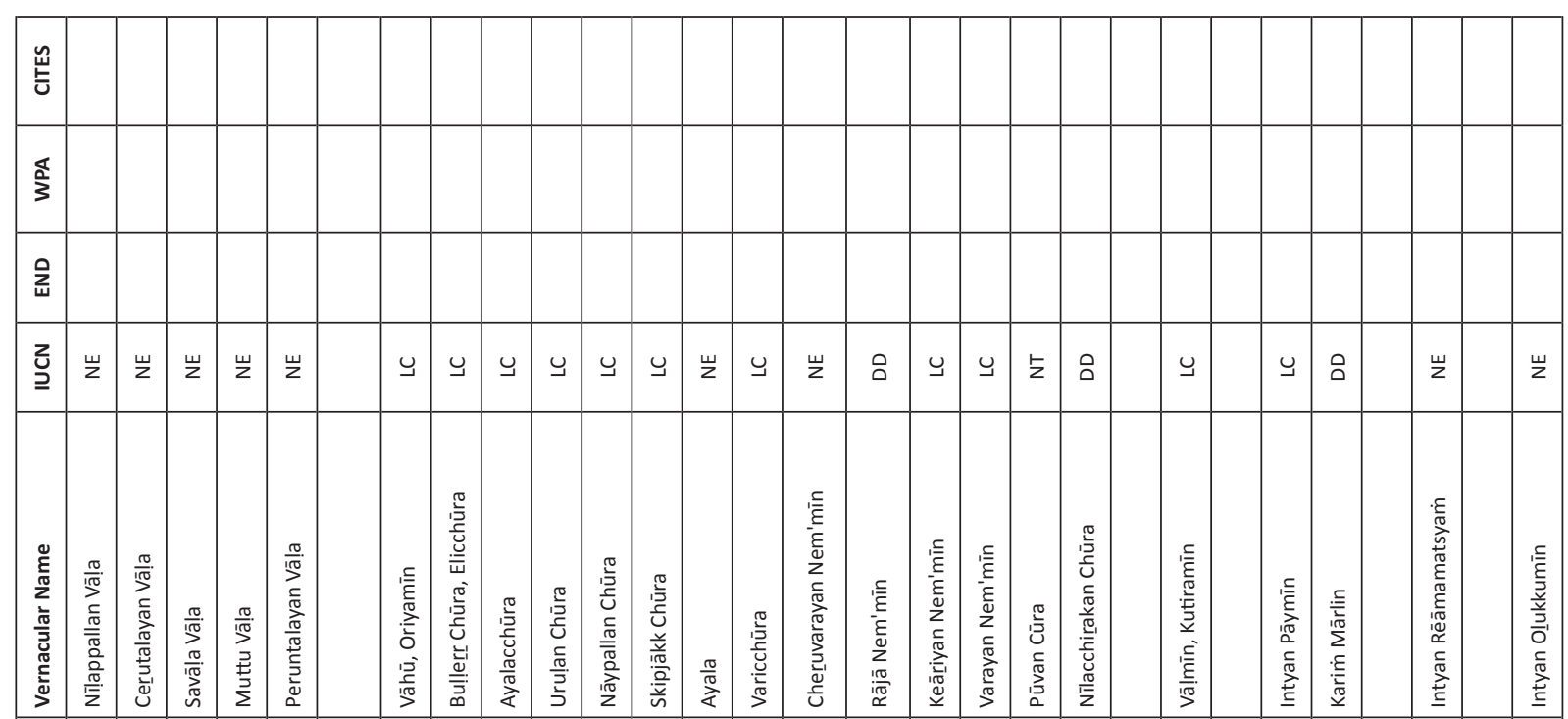

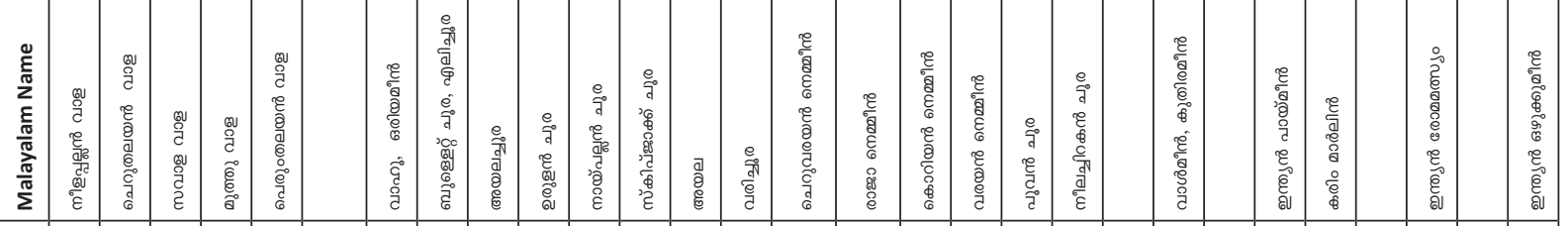

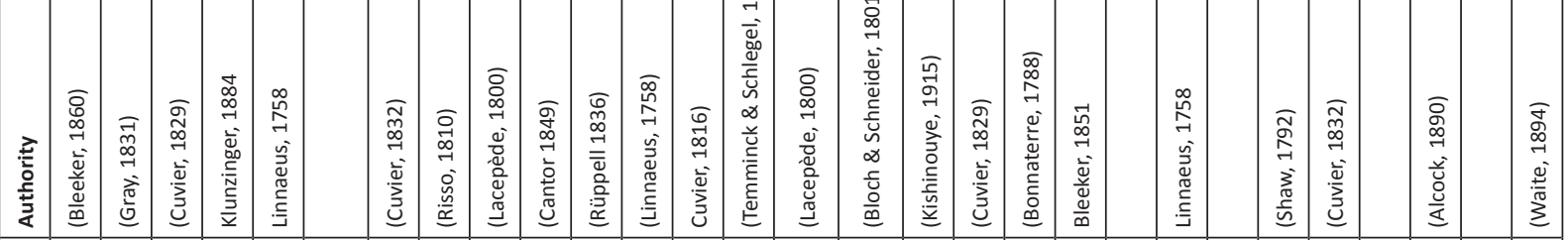

\begin{tabular}{|c|c|c|c|c|c|c|c|c|c|c|c|c|c|c|c|c|c|c|c|c|c|c|c|c|c|c|}
\hline 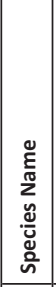 & 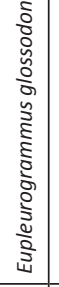 & 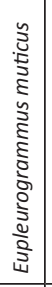 & 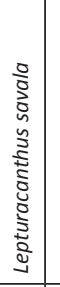 & 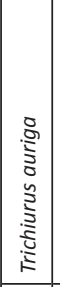 & 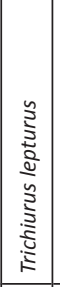 & & 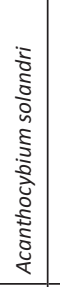 & 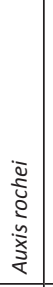 & 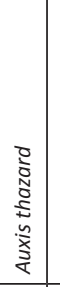 & 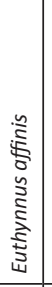 & 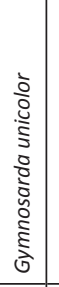 & 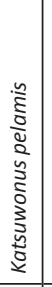 & 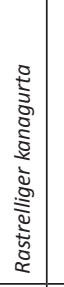 & 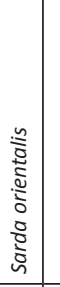 & & 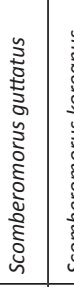 & 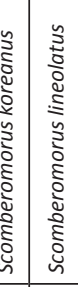 & 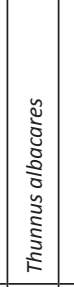 & 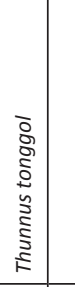 & & & 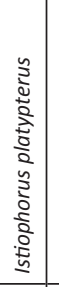 & 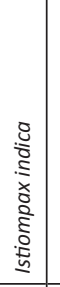 & & & 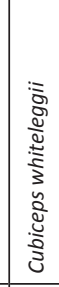 \\
\hline 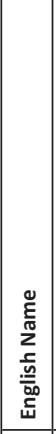 & 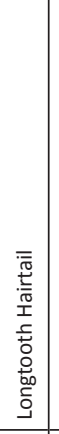 & 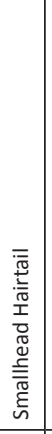 & 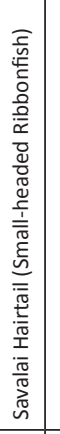 & 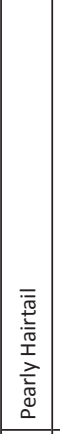 & 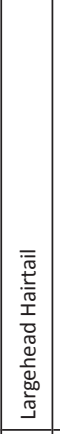 & 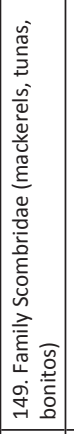 & $\begin{array}{c} \\
\stackrel{0}{0} \\
\frac{0}{m \pi} \\
3\end{array}$ & 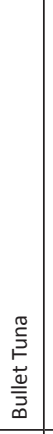 & 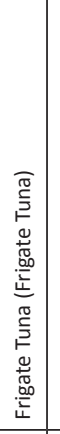 & 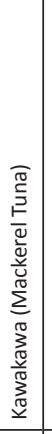 & 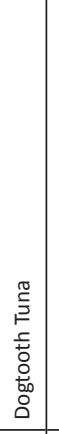 & 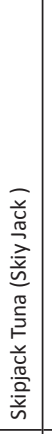 & 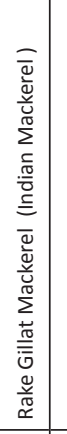 & 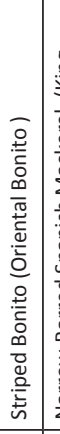 & 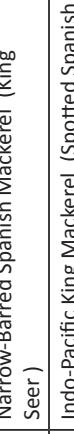 & 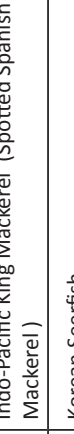 & 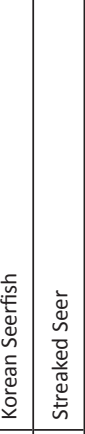 & 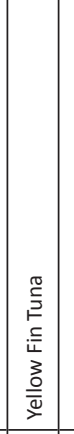 & 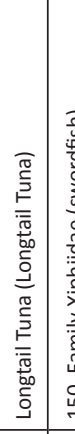 & 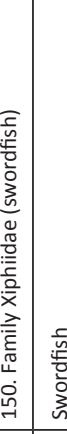 & 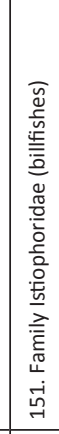 & 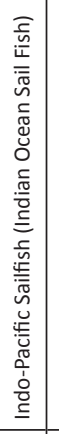 & 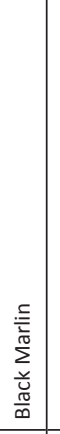 & 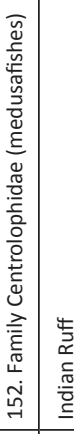 & 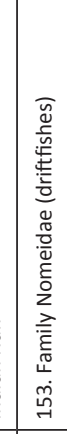 & 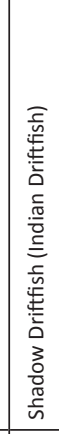 \\
\hline & 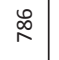 & $\stackrel{\infty}{\wedge}$ & $\begin{array}{l}\infty \\
\stackrel{\infty}{\infty} \\
\stackrel{1}{\sim}\end{array}$ & $\begin{array}{l}\stackrel{\infty}{\infty} \\
\wedge\end{array}$ & 只 & & $\vec{\Omega}$ & $\tilde{\Omega}$ & $\stackrel{m}{R}$ & مू & 号 & ঃ & 众 & $\stackrel{\infty}{\curvearrowright}$ & হి & ঃ & $\vec{\infty} \mid \tilde{\infty}$ & 离 & 志 & น & & $\begin{array}{l}\stackrel{\infty}{\infty} \\
\infty\end{array}$ & 今े & 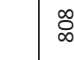 & & : \\
\hline
\end{tabular}




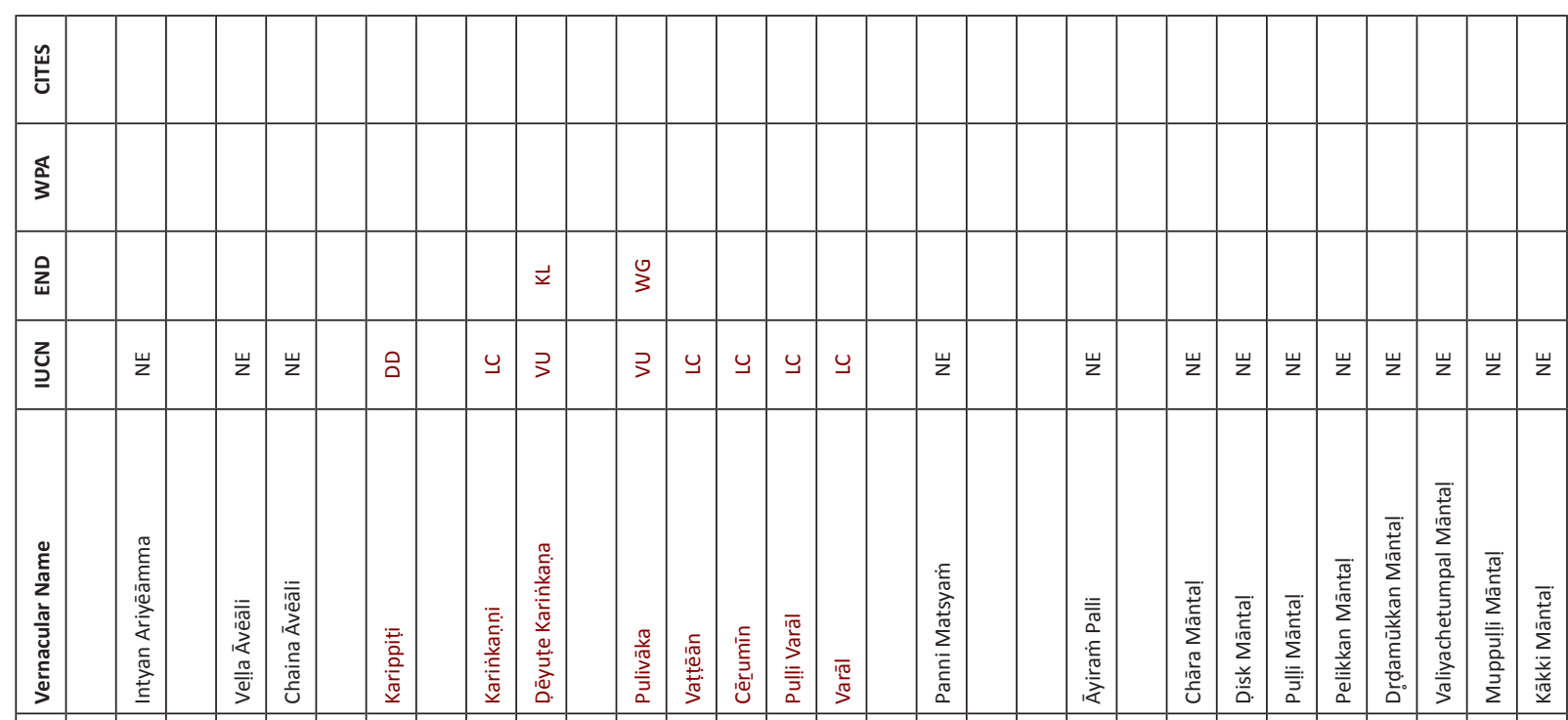

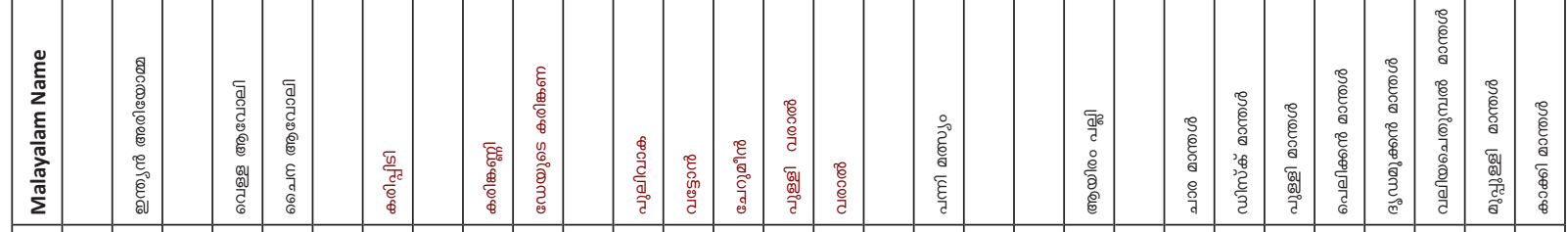

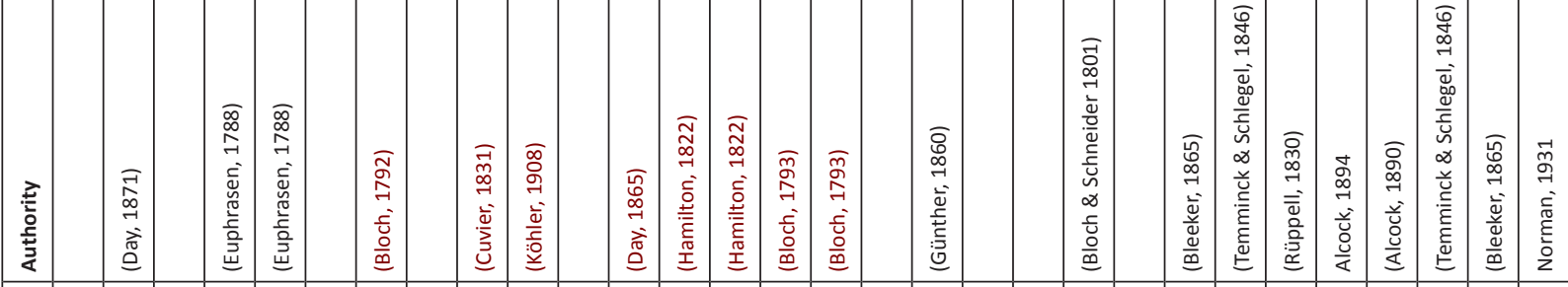

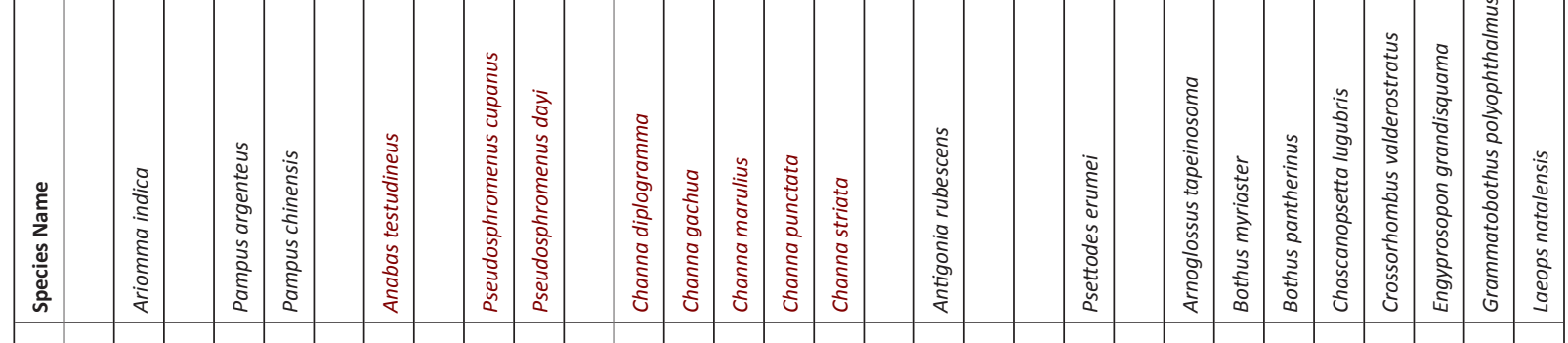

\begin{tabular}{|c|c|c|c|c|c|c|c|c|c|c|c|c|c|c|c|c|c|c|c|c|c|c|c|c|c|}
\hline$\sum_{\frac{\pi}{n}}^{\infty}$ & 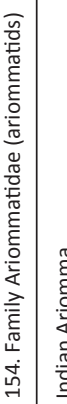 & 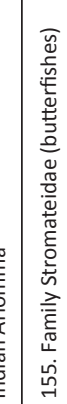 & 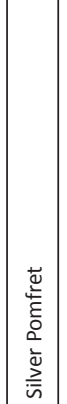 & 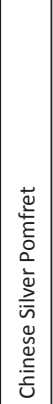 & 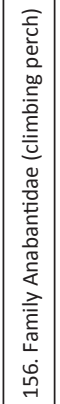 & 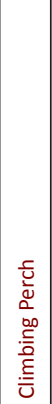 & 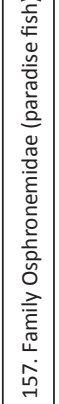 & 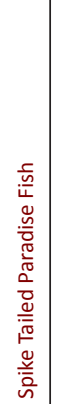 & 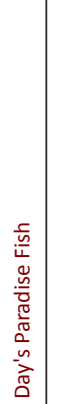 & 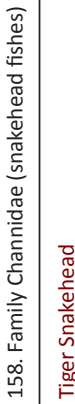 & 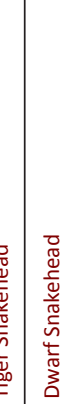 & 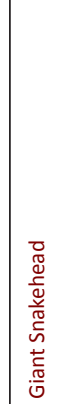 & 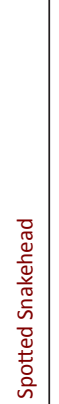 & 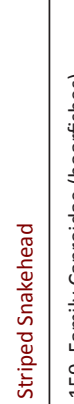 & 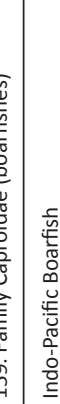 & 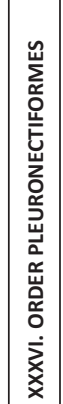 & 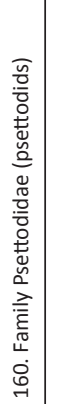 & 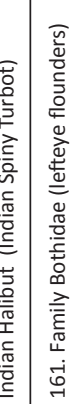 & 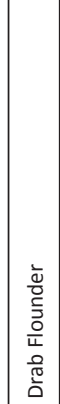 & 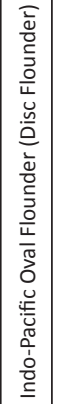 & 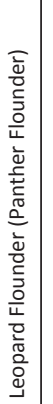 & & 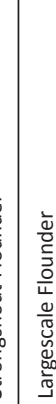 & & \\
\hline & $\stackrel{c}{\infty}$ & $\begin{array}{l}b_{1} \\
\vdots\end{array}$ & $\vec{\infty}$ & $\tilde{\infty}$ & & $\stackrel{m}{\infty}$ & & $\vec{\infty}$ & 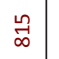 & 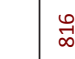 & $\begin{array}{l}\stackrel{c}{0} \\
0\end{array}$ & $\underset{\infty}{\infty}$ & $\stackrel{\vec{\infty}}{\overrightarrow{2}}$ & $\underset{\infty}{\stackrel{్}{~}}$ & $\underset{\infty}{\vec{J}}$ & & & & $\stackrel{\widetilde{\infty}}{\sim}$ & 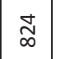 & 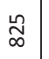 & & $\underset{\infty}{\infty}$ & $\underset{\infty}{\grave{1}}$ & \\
\hline
\end{tabular}




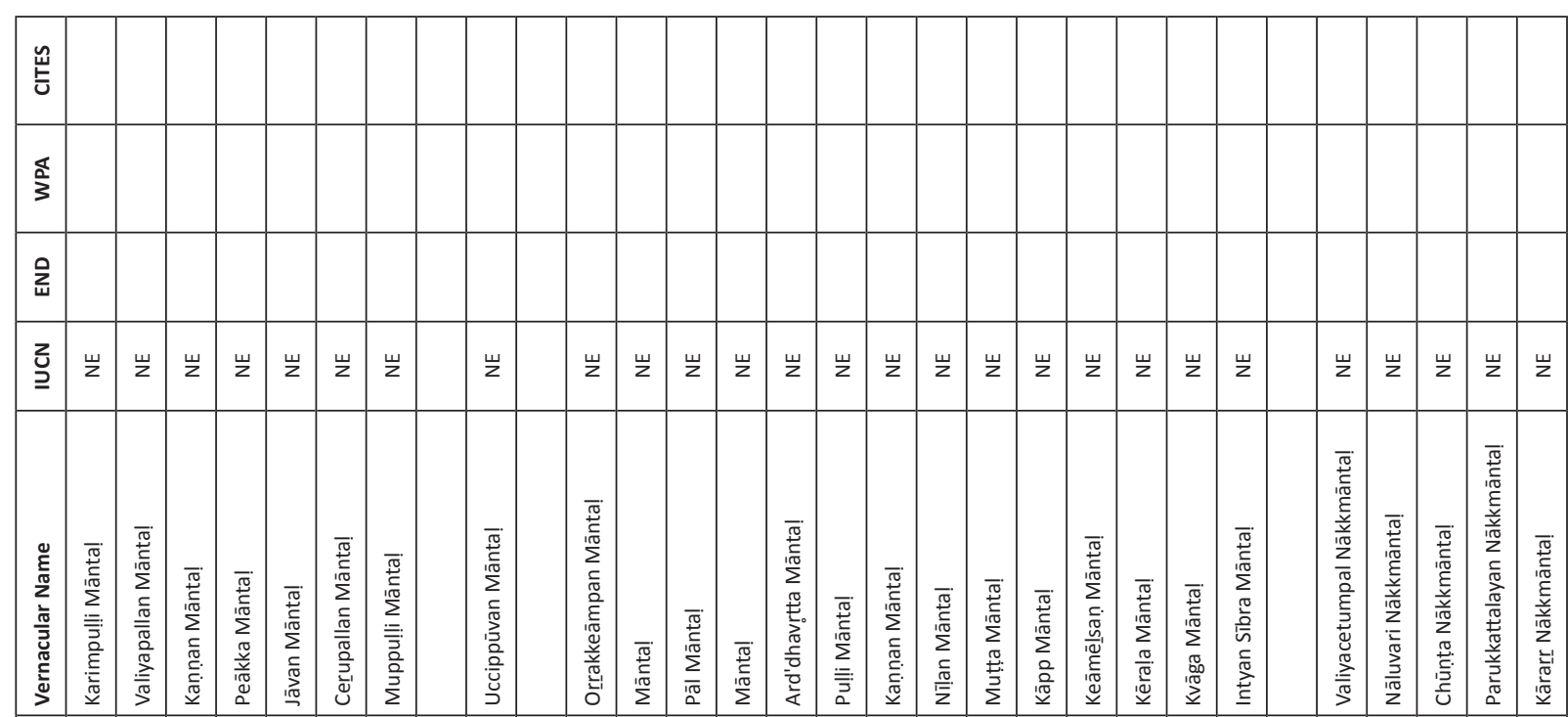

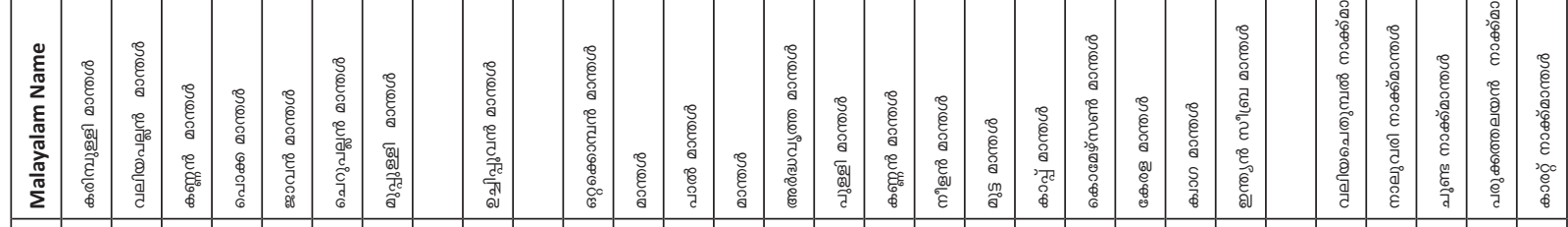

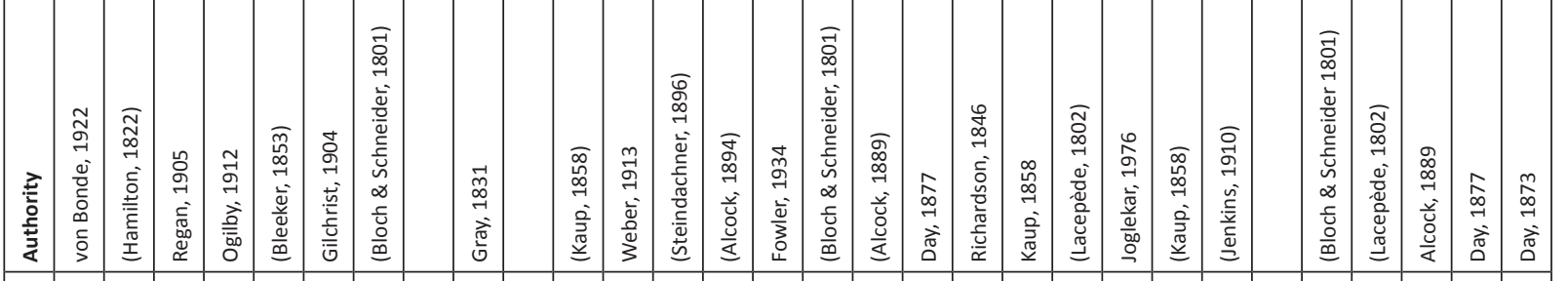

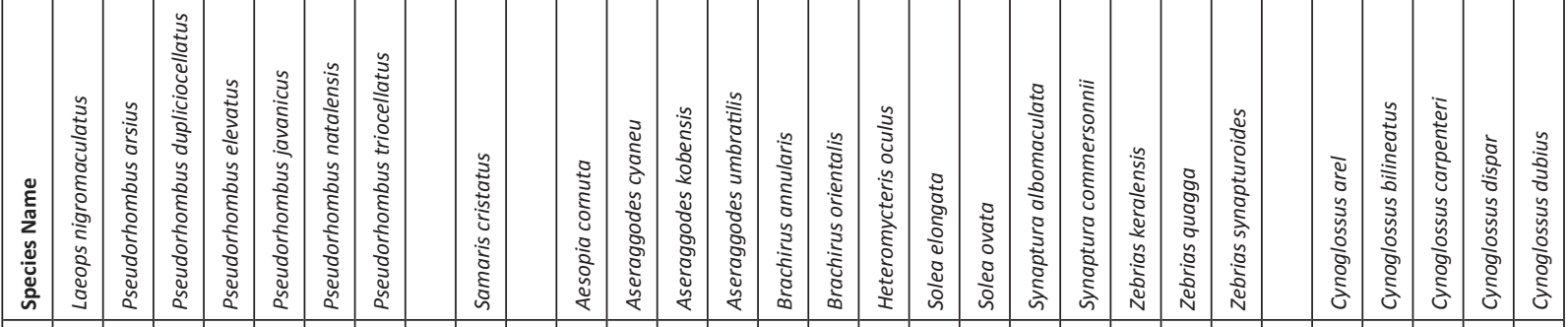

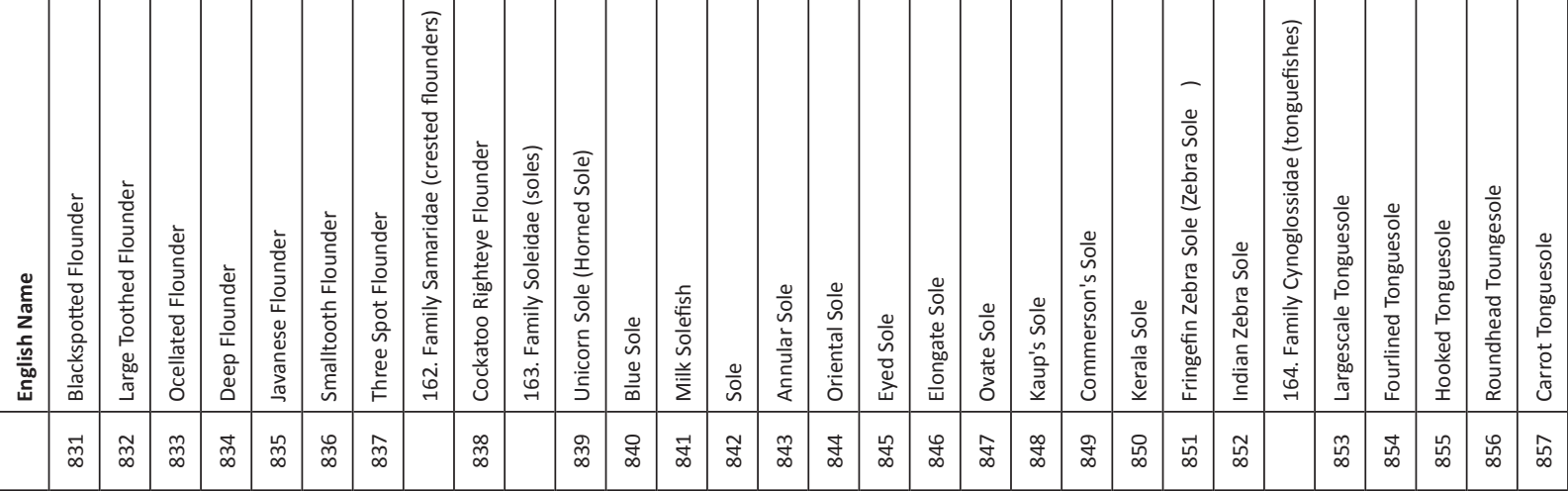




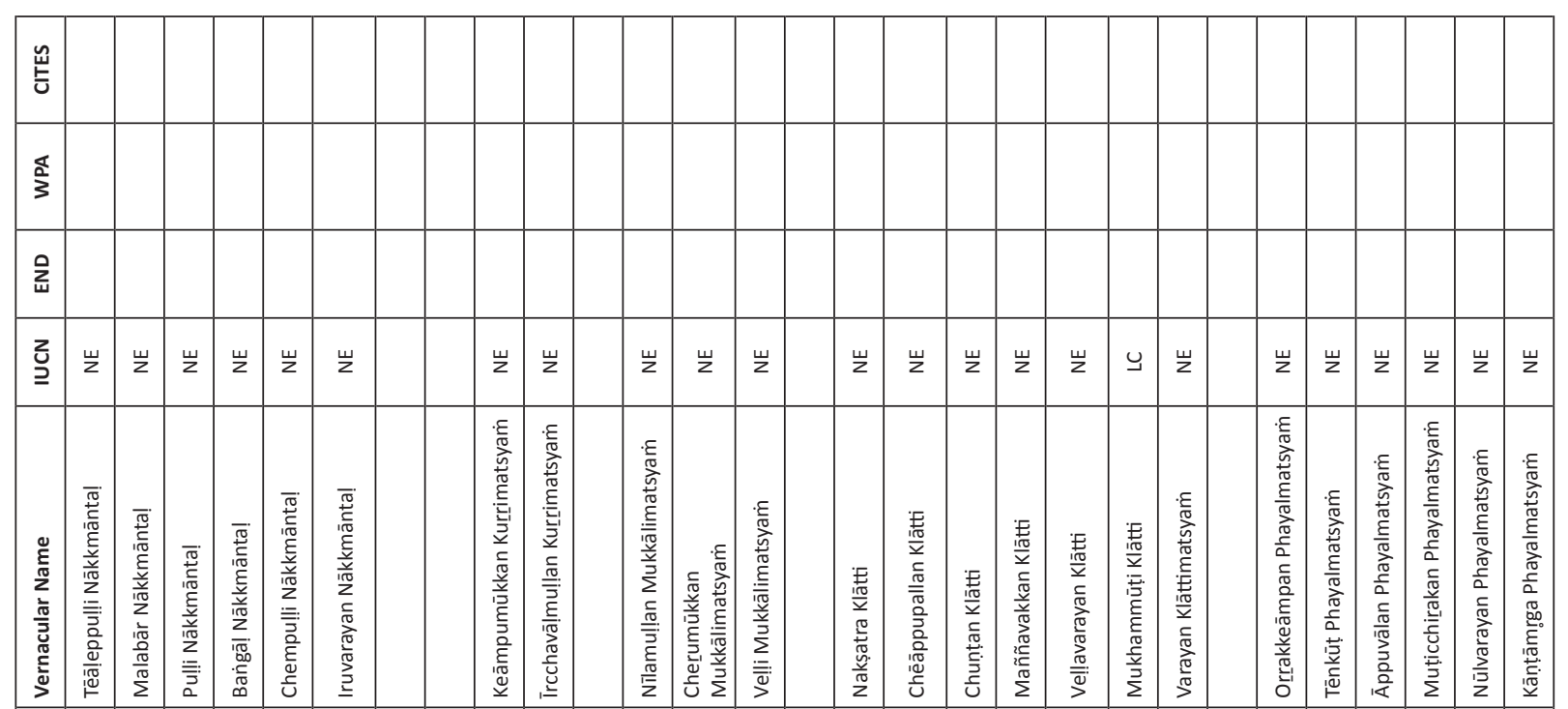

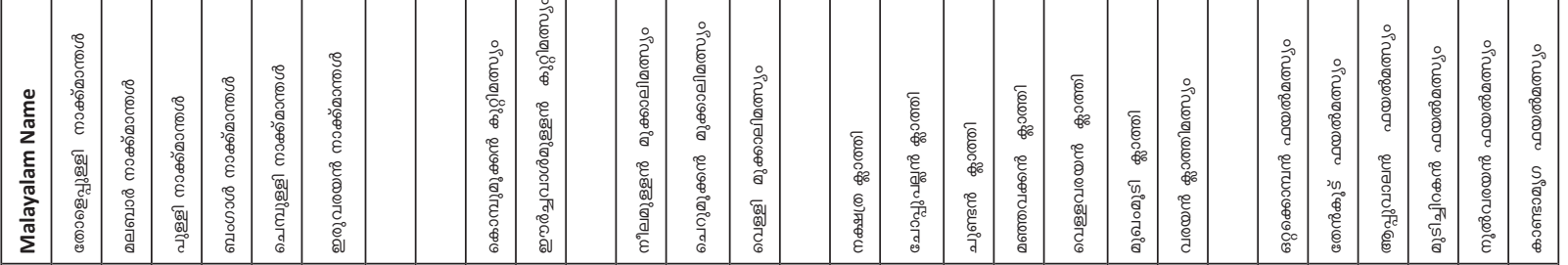

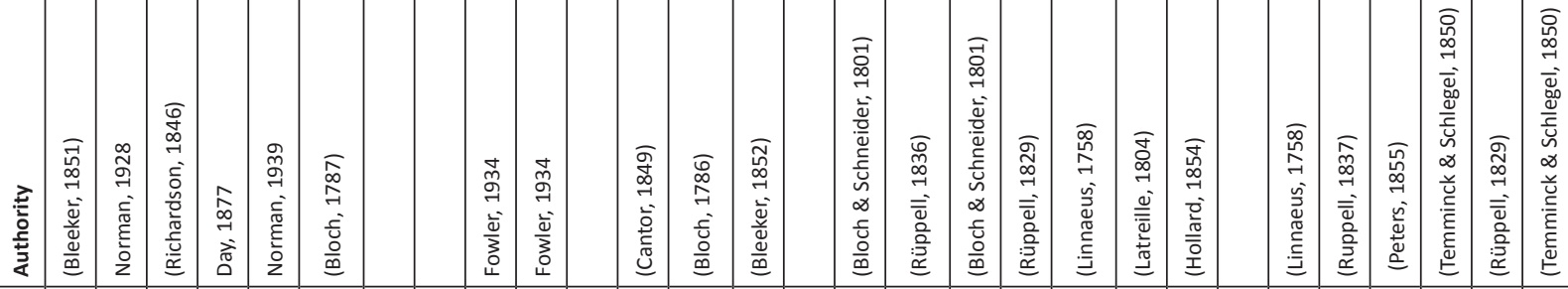

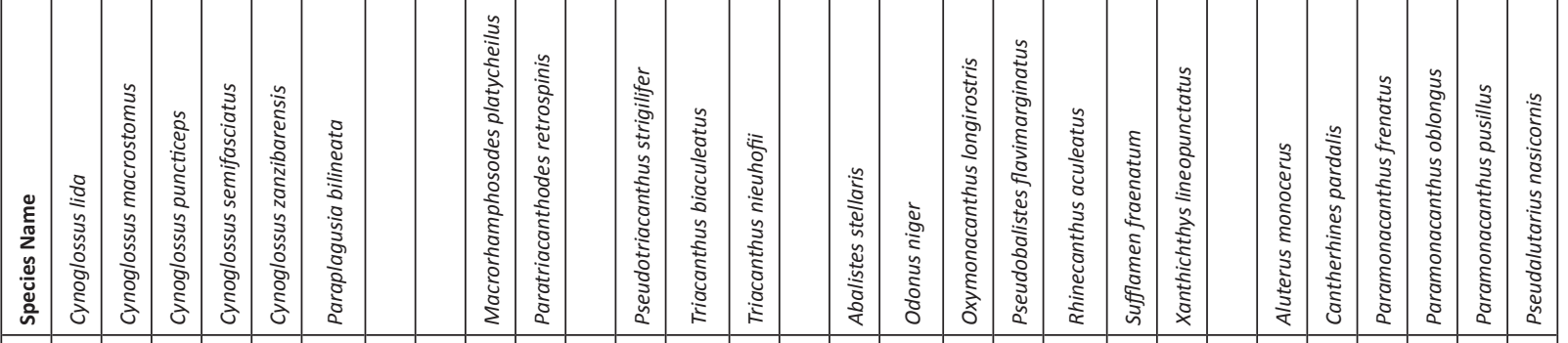

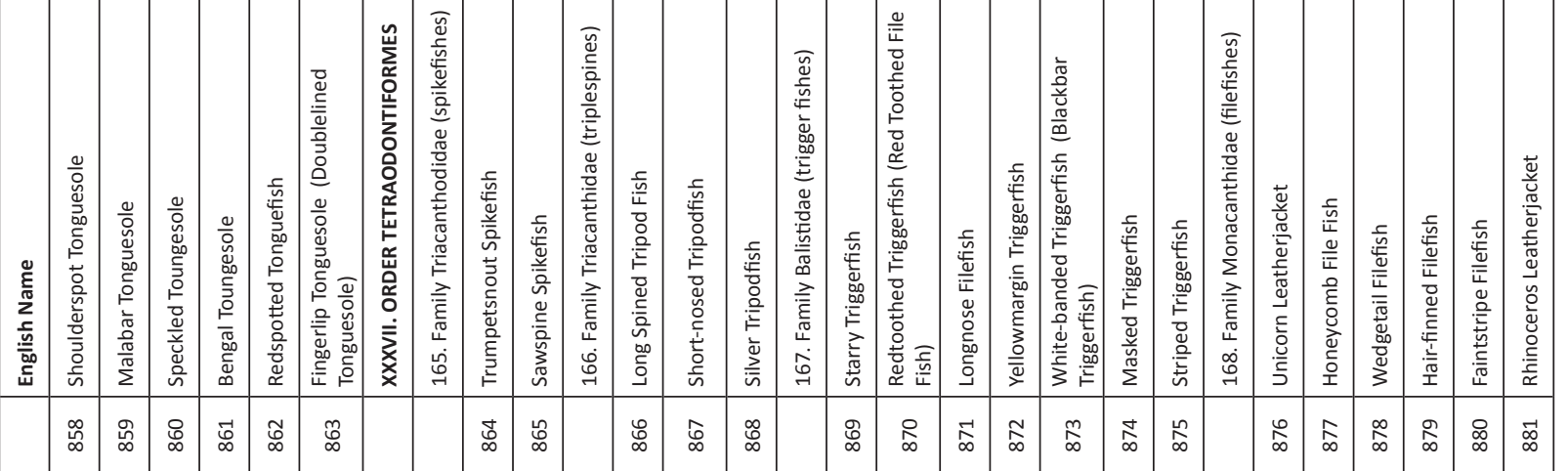




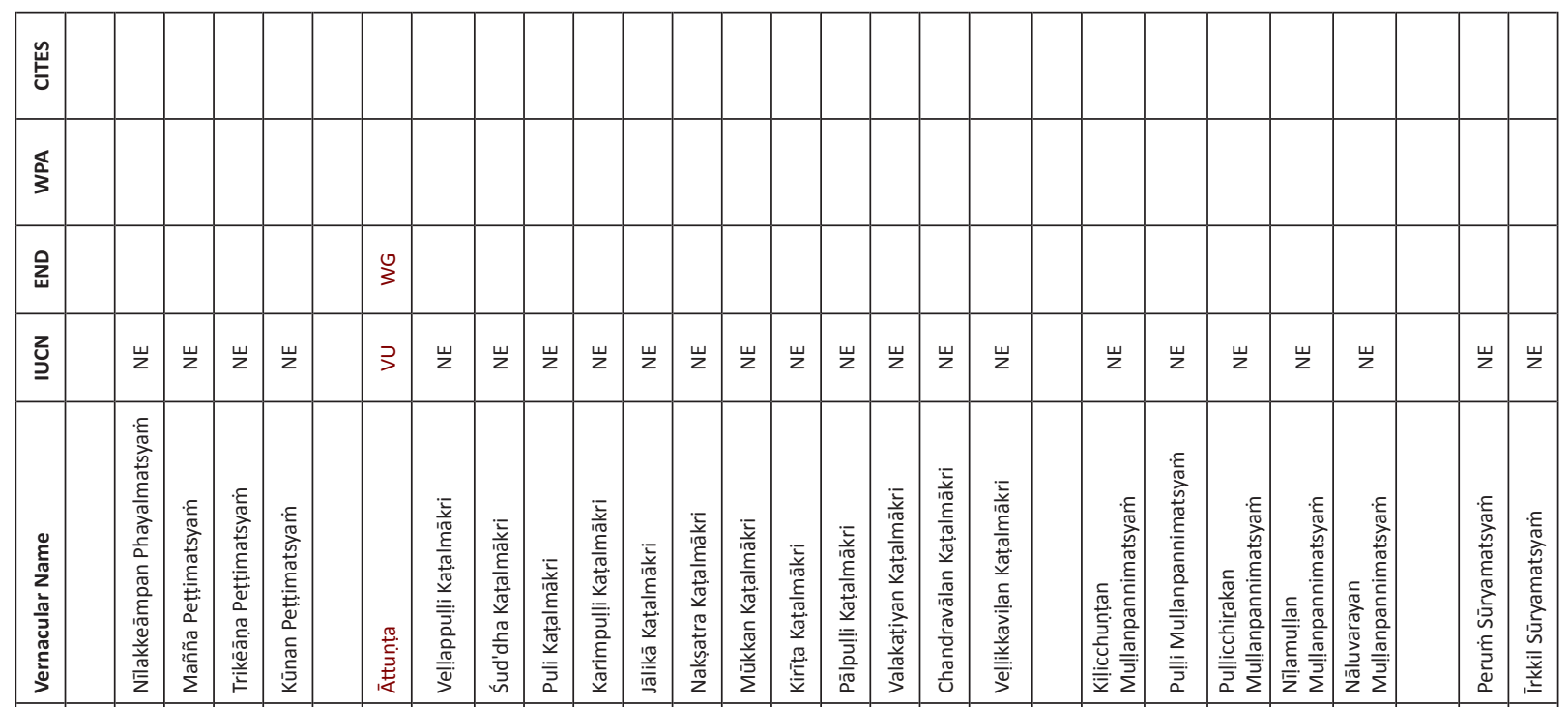

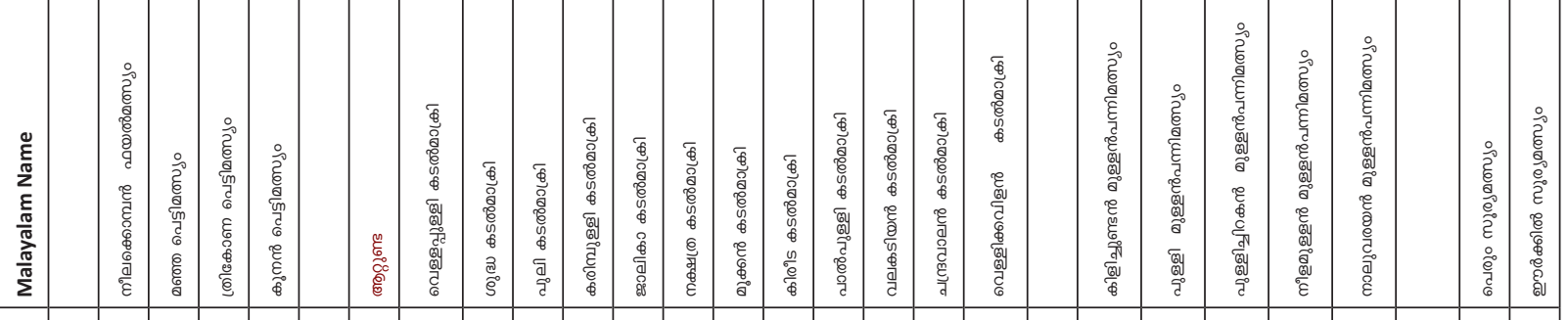

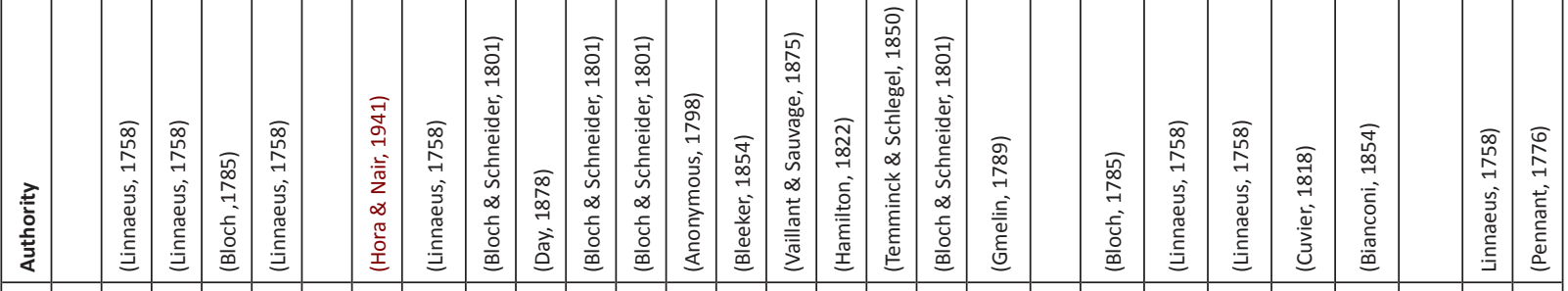

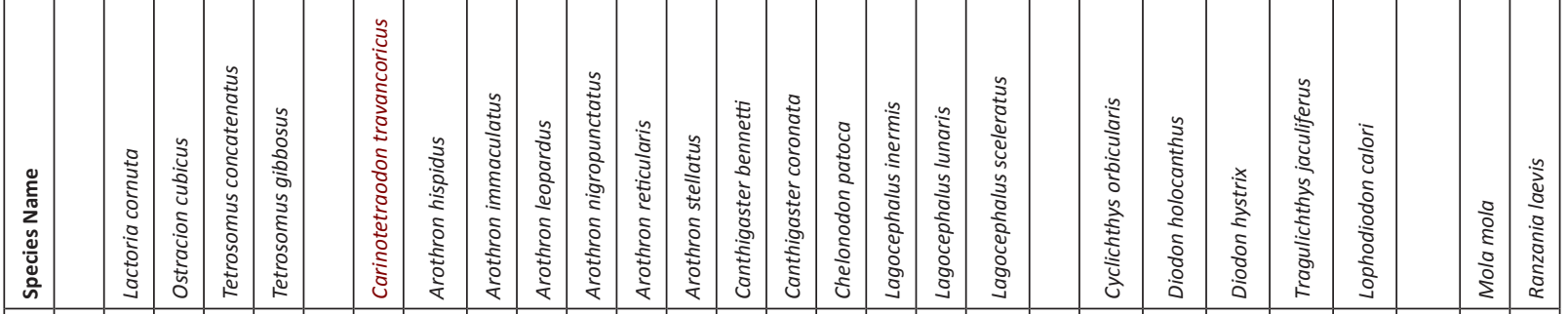

\begin{tabular}{|c|c|c|c|c|c|c|c|c|c|c|c|c|c|c|c|c|c|c|c|c|c|c|c|c|c|}
\hline 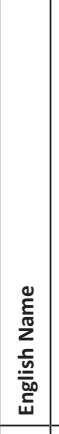 & 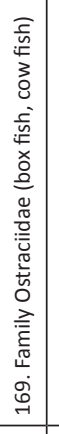 & 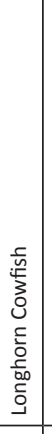 & 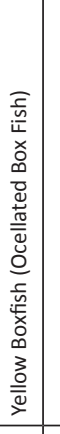 & 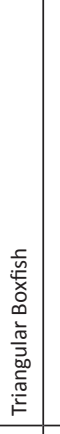 & 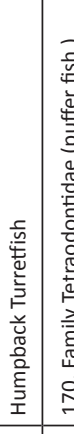 & 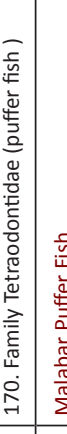 & 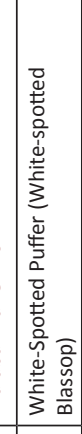 & 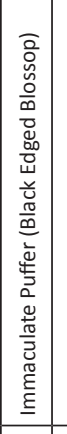 & 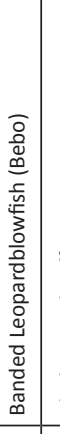 & 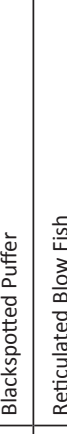 & 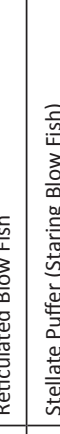 & 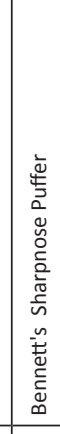 & 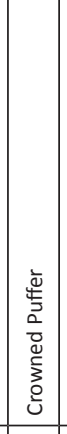 & 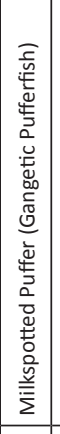 & 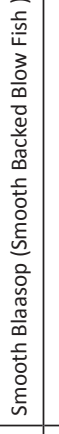 & 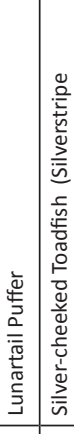 & 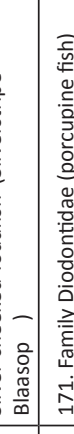 & 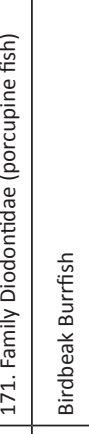 & 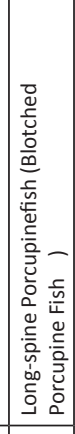 & 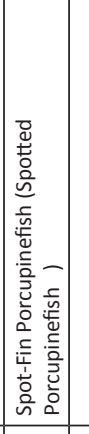 & 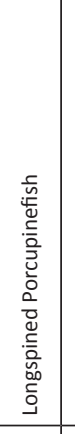 & 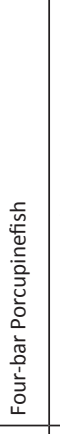 & 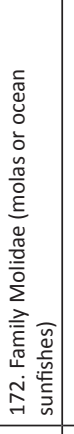 & 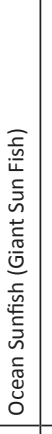 & \\
\hline & & $\underset{\infty}{\infty}$ & 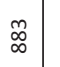 & 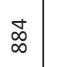 & $\begin{array}{l}\infty \\
\infty \\
\infty\end{array}$ & $\begin{array}{l}\infty \\
\infty\end{array}$ & $\begin{array}{l}\text { D } \\
\infty\end{array}$ & $\begin{array}{l}\infty \\
\infty \\
\infty\end{array}$ & $\begin{array}{l}\mathscr{D} \\
\infty\end{array}$ & ஓ & 㻤 & 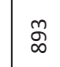 & 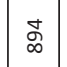 & 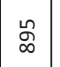 & 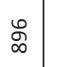 & ડ્ळ & $\begin{array}{l}\infty \\
\infty \\
\infty\end{array}$ & ஓ & ৪ু & $\vec{\circ}$ & ธั & ळ̊ & & 志 & Lू \\
\hline
\end{tabular}


${ }^{1}$ Known only from the Ashambu Hills

${ }^{2}$ Known only from the Nilgiri Biosphere Reserve

${ }^{3}$ Known only from the Periyar Watershed including the diverted waters into Tamil Nadu

${ }^{4}$ Known only from the Santhampara Hills; Previously in the genus Horalabiosa

${ }^{5}$ Known only from the Periyar River at Pooyamkutty

${ }^{6}$ Known only from the south of the Palakkad Gap

${ }^{7}$ Known only from the north of the Palakkad Gap

${ }^{8}$ Known only from the Silent Valley National Park and Chinnar Wildlife Sanctuary

${ }^{9}$ Known only from the Periyar Tiger Reserve

${ }^{10}$ Possibly an introduced population

${ }^{11}$ Known only from the rivers in Kasargod and Kannur districts

${ }^{12}$ Known only from the Kabini River

${ }^{13}$ Known only from the confluent rivers of the Vembanad Lake

${ }^{14}$ In Kerala, its distribution is restricted to the rivers in Wayanad

${ }^{15}$ Known only from the Kunthi River in Mannarkad

${ }^{16}$ Known only from the Periyar, Chalakudy and Bharathapuzha Rivers

${ }^{17}$ Known only from the Chinnar Wildlife Sanctuary

${ }^{18}$ Known only from the Silent Valley National Park

${ }^{19}$ Known only from the Silent Valley National Park and adjoining landscapes (Bhavani and Kunthipuzha)

${ }^{20}$ Known only from the Anamalai Hills

${ }^{21}$ Known only from the Santhampara Hills

${ }^{22}$ In Kerala, it is known only from Wayanad District; Previously in the genus Longischistura

${ }^{23}$ In Kerala, it is currently known only from the east flowing Kabini River

${ }^{24}$ Known only from the Periyar and Chalakudy rivers

${ }^{25}$ Known only from Thrissur District

${ }^{26}$ Known only from the Chaliyar River

${ }^{27}$ Known only from its type locality in the Manimala River

${ }^{28}$ Known only from the Aralam Wildlife Sanctuary

${ }^{29}$ Known only from Kottayam District

${ }^{30}$ The parent taxa is now considered as Tylosurus acus acus (Lacepède, 1803) and the sub species in western Indian ocean, including Indian coastal waters is represented by Tylosurus acus melanotus (Bleeker, 1850) (Bailly, 2015)

${ }^{31}$ Known only from its type locality in Kottayam; Not collected since its description

* Exotic species 


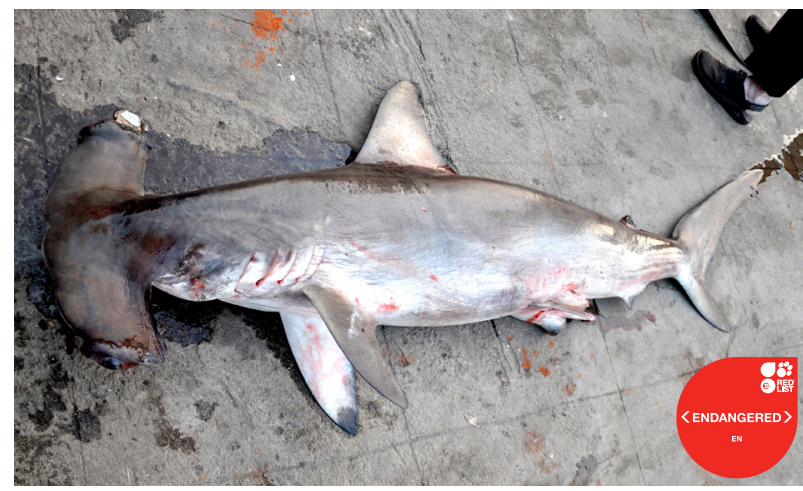

Image 1. Sphyrna lewini

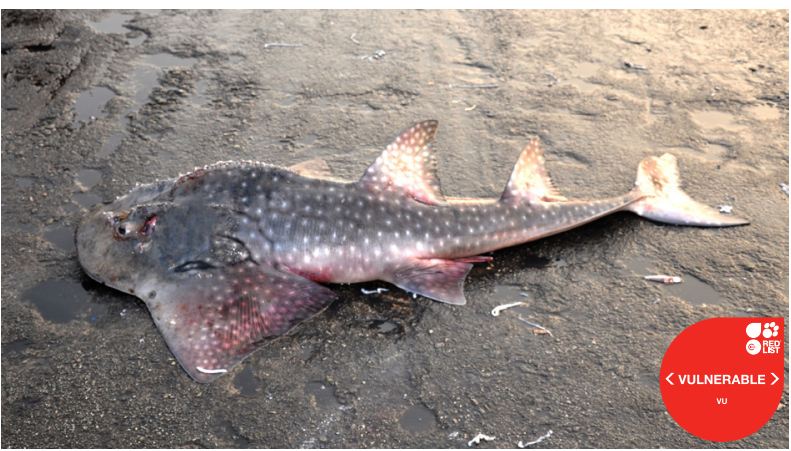

Image 3. Rhina ancylostoma

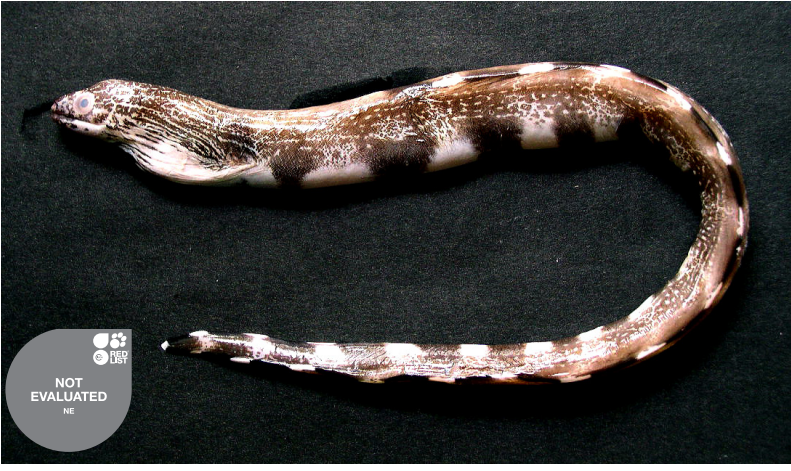

Image 5. Gymnothorax reticularis

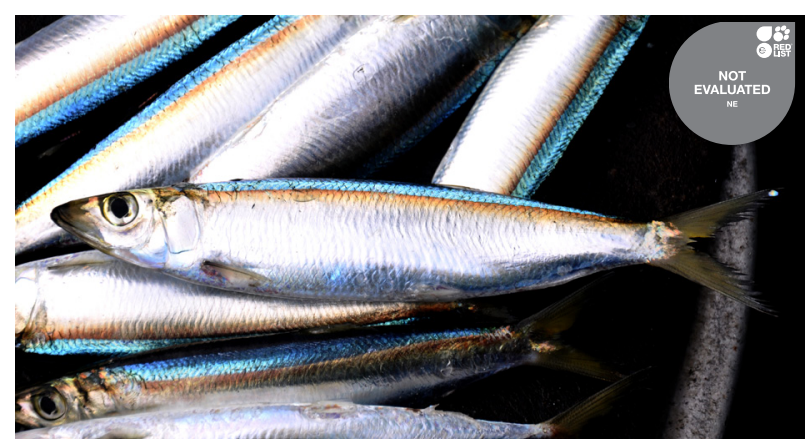

Image 7. Dussumieria acuta

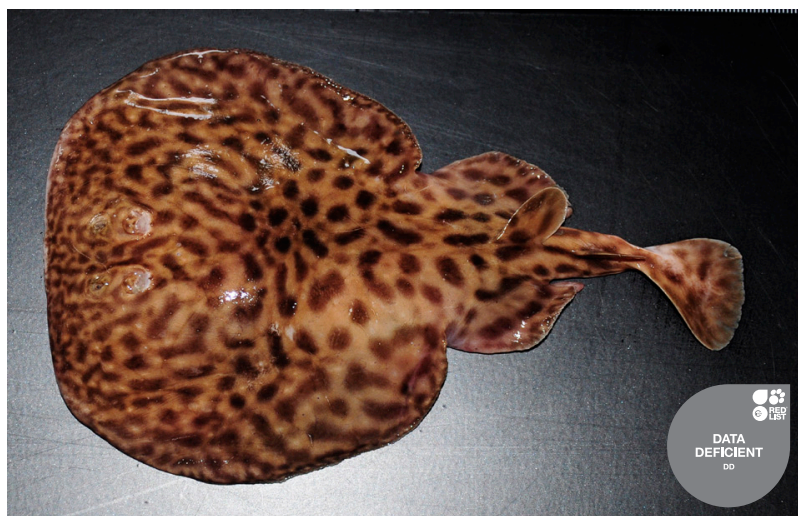

Image 2. Torpedo sinuspersici

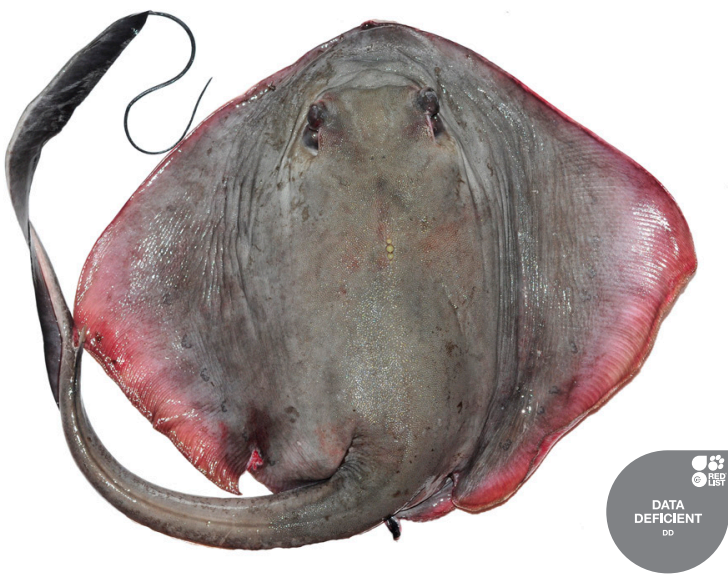

Image 4. Pastinachus sephen

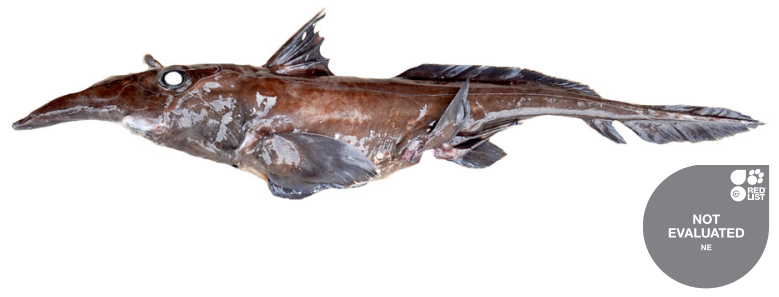

Image 6. Neoharriota pinnata

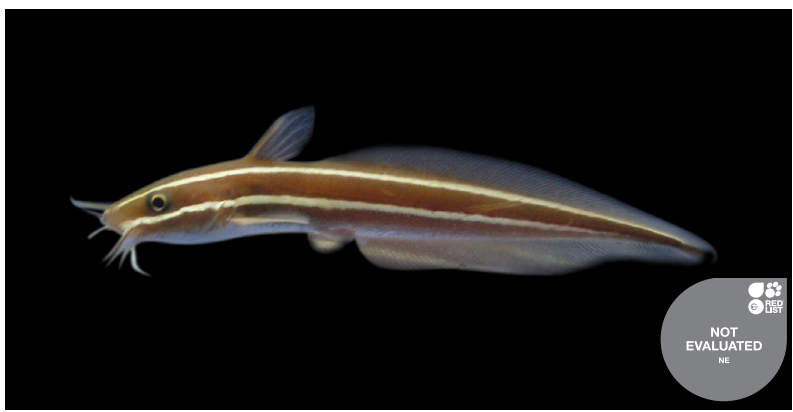

Image 8. Plotosus lineatus 

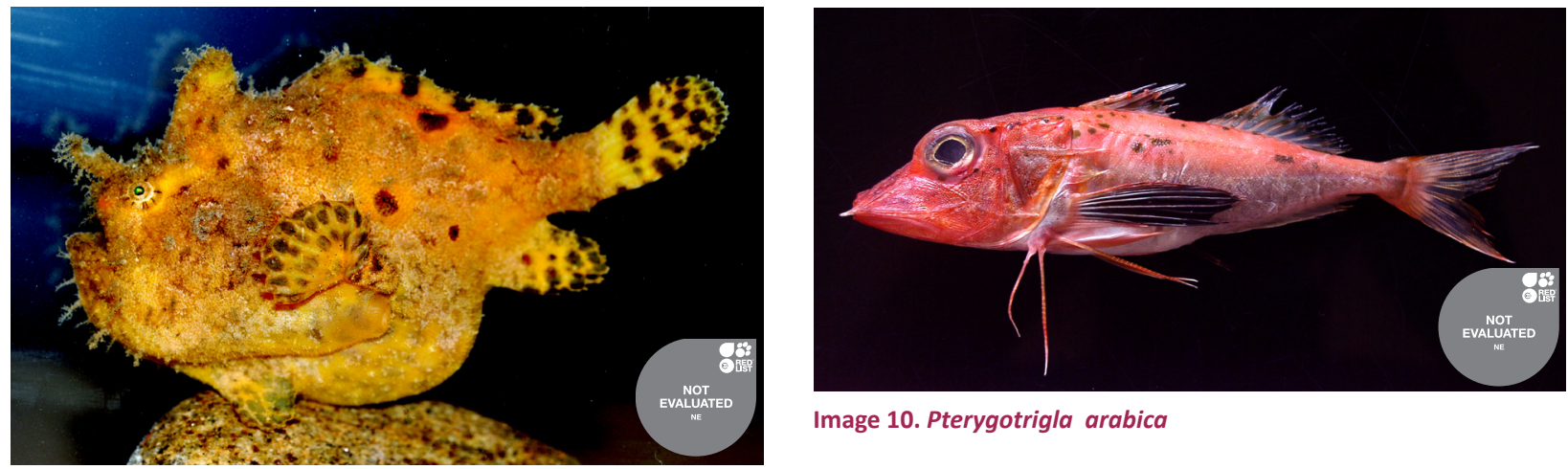

Image 10. Pterygotrigla arabica
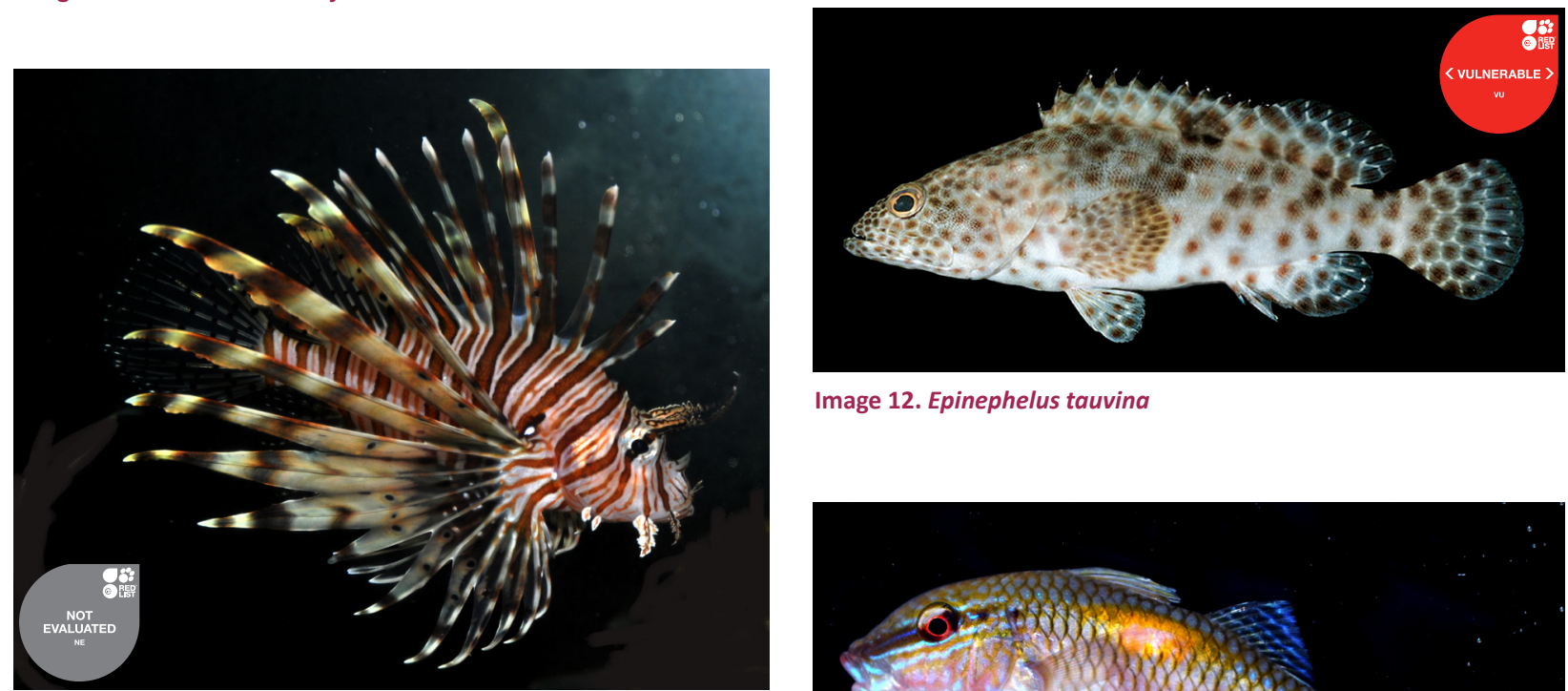

Image 12. Epinephelus tauvina

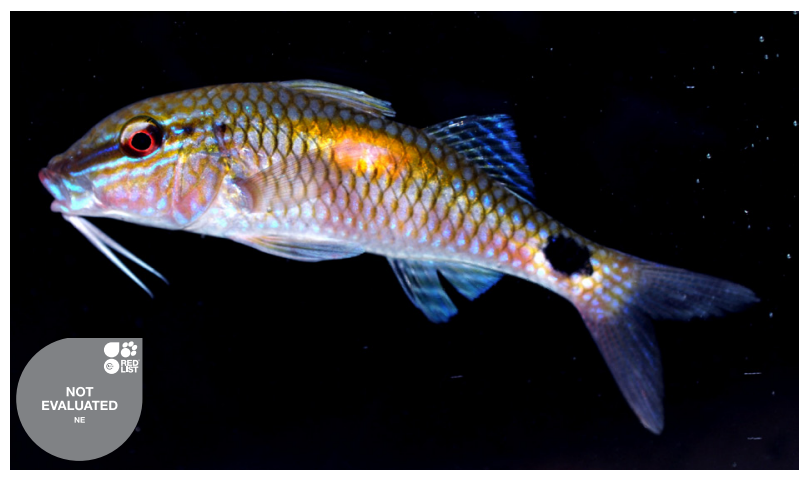

Image 13. Parupeneus indicus

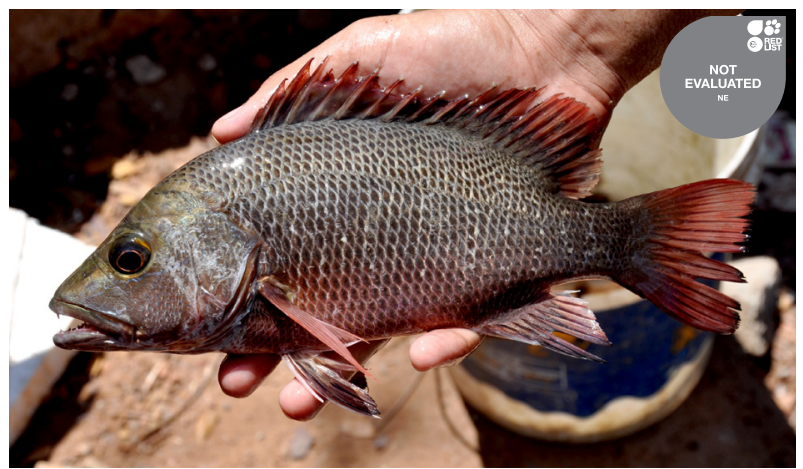

Image 16. Lutjanus argentimaculatus

Image 15. Pseudanthias marcia 


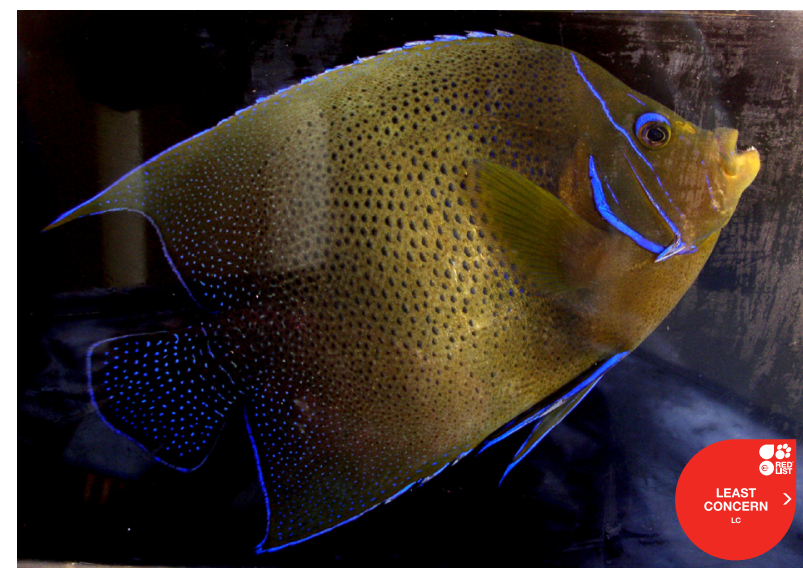

Image 17. Pomacanthus semicirculatus

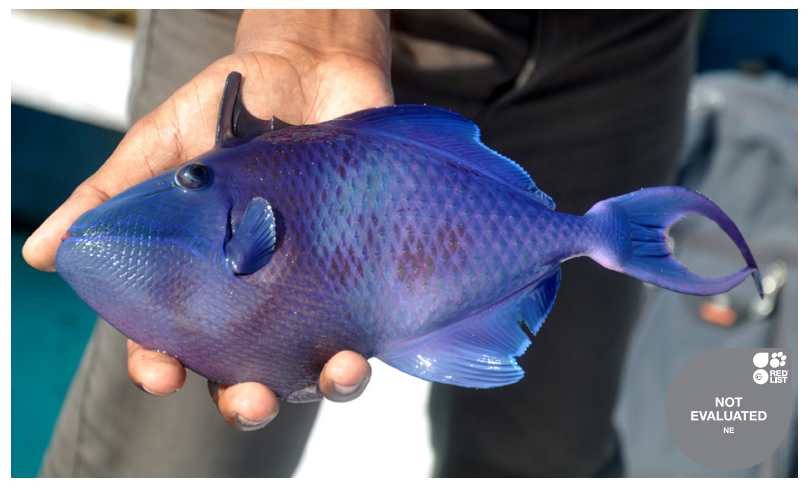

Image 19. Odonus niger

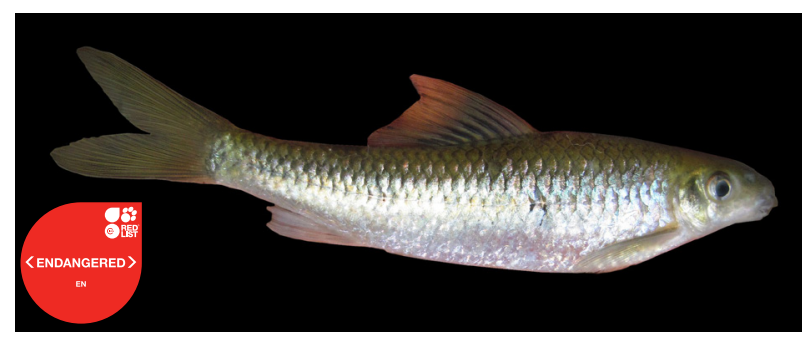

Image 21. Crossocheilus periyarensis

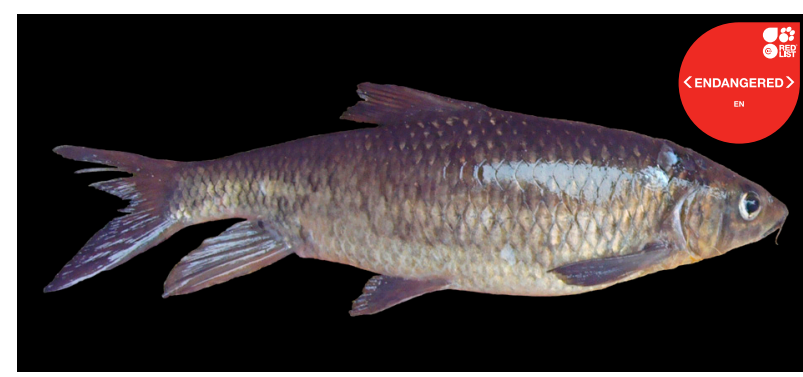

Image 23. Hypselobarbus periyarensis

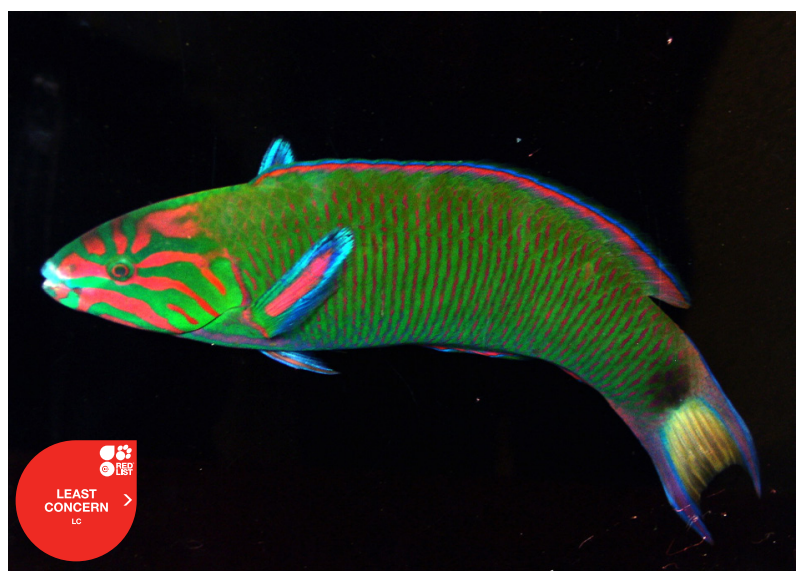

Image 18. Thalassoma lunare

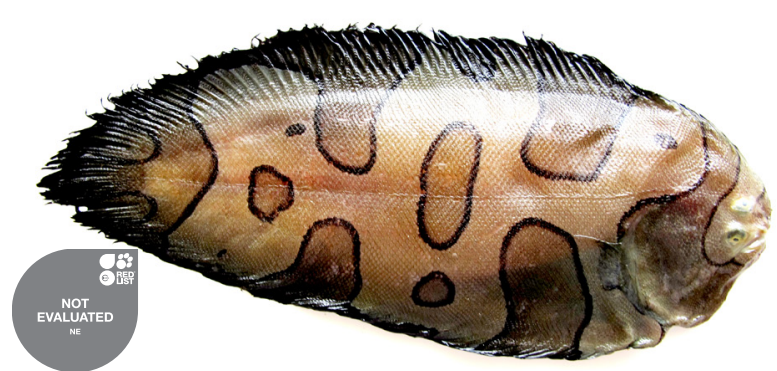

Image 20. Brachirus annularis

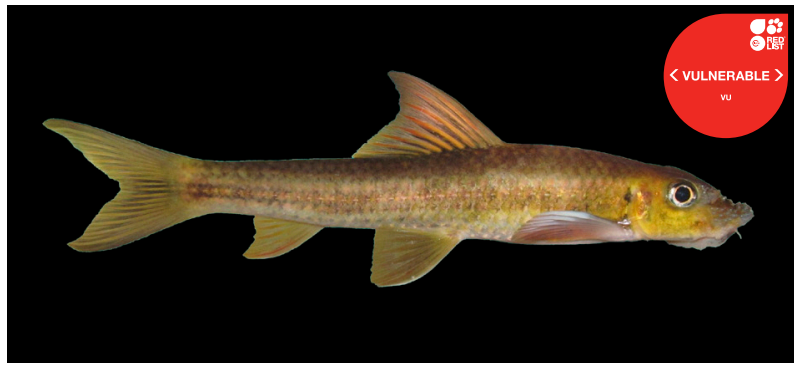

Image 22. Garra periyarensis

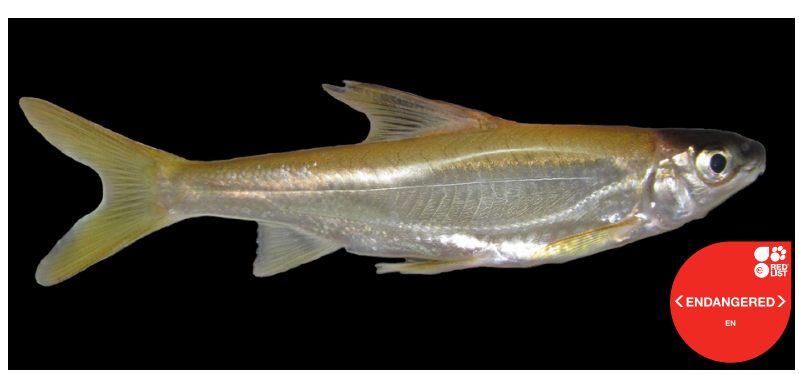

Image 24. Lepidopygopsis typus 


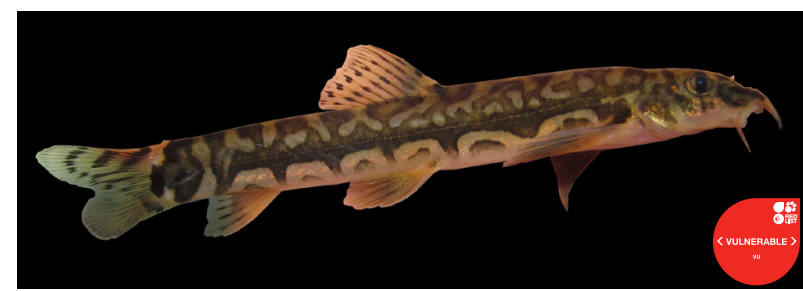

Image 25. Nemacheilus menonii

of Western Ghats with Special Reference to Fish Community. US Fish and Wildlife Service \& Bombay Natural History Society.

Day, F. (1865a). The Fishes of Malabar. London. i-xxxii + 1-293, 20pls.

Day, F. (1865b). On the fishes of Cochin, on the Malabar coast of India. Part I. Acanthopterygii. Proceedings of the Zoological Society of London 1865 (pt 1) (art. 1): 2-40; http://dx.doi. org/10.1111/j.1469-7998.1865.tb02299.x

Day, F. (1865c). On the fishes of Cochin, on the Malabar Coast of India. Part II. Anacanthini. Proceedings of the Zoological Society of London 1865 (pt 1): 286-318; http://dx.doi.org/10.1111/j.1469-7998.1865. tb02337.x

Day, F. (1867a). On the fishes of the Neilgherry Hills and rivers around their bases. Proceedings of the Zoological Society of London 1867 (pt 2): 281-302.

Day, F. (1867b). On some fishes from the Wynaad. Proceedings of the Zoological Society of London 1867 (pt 2): 347-350.

De Beaufort, L.F. \& J.C. Briggs (1962). The Fishes of Indo-Australian Archipelago. E.J. Brill, Leiden, 11, 481pp.

De Beaufort, L.F. \& W.M. Chapman (1951). The Fishes of IndoAustralian Archipelago. E.J. Brill, Leiden, 9, 484pp.

Gopi K.C. \& S.S. Mishra (2015). Diversity of Marine Fish of India, pp 171-193. In: Venkataraman K. \& C. Sivaperuman (eds.). Marine Faunal Diversity in India. Taxonomy, Ecology and Conservation. Elsevier Publ., Amsterdam.

Gopi, K.C (2000). Freshwater fishes of Kerala State, pp. 5676. In: Ponniah, A.G. \& A. Gopalakrishnan (eds.). Endemic Fish Diversity of Western Ghats. NBFGR-NATP, India.

Günther, A.C (1864). Catalogue of the fishes in the British Museum. Catalogue of the Physostomi, containing the families Siluridae, Characinidae, Haplochitonidae, Sternoptychidae, Scopelidae, Stomiatidae in the collection of the British Museum, v. 5: i-xxii + 1-455pp.

Hora, S.L. \& N.C. Law (1941). The freshwater fishes of Travancore. Records of Indian Museum 43: 233-256.

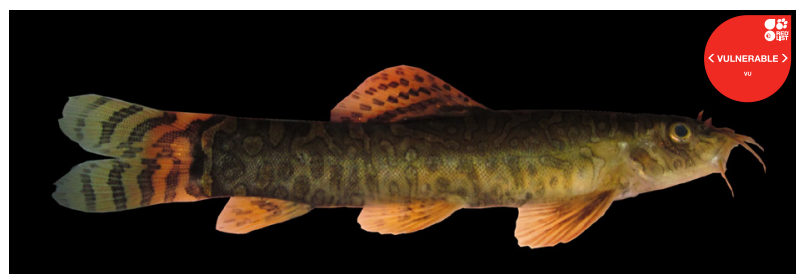

Image 26. Mesonoemacheilus periyarensis

Jerdon, T.C. (1849). On the freshwater fishes of southern India. Madras Journal of Literature and Science 15(2): 302-346.

Kapoor, D., R. Dayal \& A.G. Ponniah (2002). Fish Biodiversity of India. National Bureau of Fish Genetic Resources Lucknow, India, 775pp.

Kurup, B.M., K.V. Radhakrishnan \& T.G. Manojkumar (2004). Biodiversity Status of Fishes Inhabiting Rivers of Kerala (South India) With Special Reference to Endemism, Threats and Conservation Measures. In: Welcomme, R.L \& T. Petr. (eds). Proceedings of the Second International Symposium on the Management of Large Rivers for Fisheries 2 (LARS2), Cambodia, 310pp.

Menon, A.G.K. (1999). Checklist of Fresh Water Fishes of India. Records of the Zoological Survey of India, No.175, 366pp.

Pillai, R.S.N. (1929). A list of fishes taken in Travancore from 19011915. Journal of the Bombay Natural History Society 33(2): 347-379.

Raghavan, R., N. Dahanukar, J.D.M. Knight, A. Bijukumar, U. Katwate, K. Krishnakumar, A. Ali \& S. Philip (2014). Predatory journals and Indian ichthyology. Current Science 107: 740-742.

Raj, B.S. (1941a). A new genus of Schizothoracine fishes from Travancore, south India. Records of the Indian Museum (Calcutta) 43(2): 209-214.

Raj, B.S. (1941b). Two new Cyprinid fishes from Travancore, South India, with remarks on Barbus (Puntius) micropogon Cuv. and Val. Records of the Indian Museum (Calcutta) 43(3): 375-386.

Silas, E.G. (1951). Fishes from the high range of Travancore. Journal of the Bombay Natural History Society 50(2): 323-330.

Talwar, P.K. \& A.G. Jhingran, (1991). Inland fishes of India and adjacent countries. Vol. I and II. Oxford and IBH Publ., New Delhi, 1158pp.

Talwar, P.K. \& R.K. Kacker (1984). Commercial Sea Fishes of India. Hand Book. Zoological Survey of India, Calcutta.

Weber, M. \& L.F. de Beaufort (1916-36). The Fishes of Indo-Australian Archipelago. E. J. Brill, Leiden, 3 (1916): 455pp; 4 (1922): 410pp; 5 (1929): 458pp; 6 (1931): 448pp; and 7 (1936): 607pp.

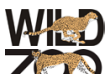

